\title{
Automated fabrication of mobility aids (AFMA): Below-knee CASD/CAM testing and evaluation program results
}

\author{
Vern L. Houston, PhD, CPO; Ernest M. Burgess, MD; Dudley S. Childress, PhD; Hans R. Lehneis, PhD, \\ CPO; Carl P. Mason, MSBE; Mary Anne Garbarini, MA, PT; Kenneth P. LaBlanc, BA, CPO; David A. \\ Boone, BA, CPO; Richmond B. Chan, PhD; John H. Harlan, MSME; Michael D. Brncick, BA, CPO \\ Department of Rehabilitation Medicine, New York University Medical Center, New York, NY 10016; \\ VA Medical Center, New York, NY 10010; Prosthetics Research Study, Seattle, WA 98122; \\ Prosthetics Research Laboratory, Northwestern University, Chicago, IL 60611; VA Lakeside Medical \\ Center, Chicago, IL 60611
}

\begin{abstract}
In 1988 the Department of Veterans Affairs Rehabilitation Research and Development Service, under the directorship of Margaret J. Giannini, M.D., began a nationally directed computer-aided design and computeraided manufacturing (CAD/CAM) research program for the Automated Fabrication of Mobility Aids (AFMA). Under this program CAD/CAM research and development centers were established at the Prosthetics Research Study in Seattle, WA; at Northwestern University and the VA Lakeside Medical Center in Chicago, IL; and at the VA Medical Center and New York University Medical Center in New York, NY. These three centers conducted a collaborative program: $(a)$ to introduce $\mathrm{CAD} / \mathrm{CAM}$ technologies to prosthetists, physicians, therapists, and rehabilitation health care professionals in the United States; $(b)$ to evaluate the feasibility of using CAD/CAM systems in clinical prosthetics settings; $(c)$ to test and evaluate the University College London-Bioengineering Center's and the University of British Columbia-Medical
\end{abstract}

Address all correspondence and requests for reprints to: Vern $\mathrm{L}$. Houston, PhD, Rehabilitation Engineering, Department of Veterans Affairs, 252 Seventh Ave, New York, NY 10001.

V.L. Houston, C.P. Mason, K.P. LaBlanc, and M.A. Garbarini are affiliated with the VA Medical Center, New York, NY and New York University Medical Center; E.M. Burgess, D.A. Boone, and J.H. Harlan are with Prosthetic Research Study, Seattle, WA; D.S. Childress, R.B. Chan, and M.D. Brncick are affiliated with Northwestern University and the VA Lakeside Medical Center, Chicago, IL; and H.R. Lehneis is with Lehneis Orthotics and Prosthetics Association, Ltd., New York, NY.
Engineering Resource Unit's respective systems for the computer-aided design and computer-aided manufacture of prosthetic sockets (CASD/CAM) for below-knee amputees; and, $(d)$ to obtain quantitative data for refinement of the CASD/CAM systems tested, and for the development of new, enhanced, more efficacious, and expedient systems.

Key words: below-knee amputees, clinical testing and evaluation, computer-aided design, computer-aided manufacturing, computer-aided socket design, prostheses.

\section{INTRODUCTION}

Although the idea of applying computer-aided design and computer-aided manufacturing (CAD/ CAM) technologies in prosthetics was conceived in the early $1960 \mathrm{~s}^{1}$, it did not reach fruition until 1983 , when a prototype system was demonstrated at the Fourth World Congress of the International Society for Prosthetics and Orthotics (ISPO) in London, England. The system demonstrated there was developed under the collaborative efforts of the Univer-

\footnotetext{
${ }^{1}$ Correspondence between James Foort and Colin McLaurin, Sc.D. outlining concepts and steps for development of a lower limb prosthetics CAD/CAM system, dated June 1964, Vancouver, BC, Canada.
} 
sity of British Columbia-Medical Engineering Resource Unit (UBC-MERU), the University of Toronto, and the University College LondonBioengineering Center (UCL-BC). It consisted of a prototype optical scanner developed at the University of Toronto, a below-knee computer-aided socket design (BK CASD) program developed at the UBC-MERU, and a computer-numerically-controlled (CNC) milling machine and plastic thermoforming machine for the automated manufacture of BK CASD sockets developed at the UCL-BC (1). Subsequent to this seminal demonstration of CAD/CAM technologies in Prosthetics, $\mathrm{CAD} / \mathrm{CAM}$ research and development gained considerable momentum, until, at present, there are ongoing prosthetics CAD/CAM research and development programs in the United States, Canada, England, Scotland, Japan, Belgium, and Sweden.

The United States Department of Veterans Affairs Rehabilitation Research and Development Service (DVA RR\&DS) has had a long and active interest in CAD/CAM technologies and their application in prosthetics and orthotics. In 1983, after the ISPO Fourth World Congress, the DVA RR\&DS held a meeting of key DVA researchers to assess the benefits and status of prosthetics-orthotics CAD/ CAM. Pursuant to this, in 1985 the DVA RR\&DS sponsored a national workshop to continue assessment of the potential of CAD/CAM technologies in prosthetics and orthotics, and to help chart the role of the DVA in future research and development of these technologies in the United States (2). In addition, in 1984 the DVA RR\&DS began funding research on the "Automated Fabrication of Lower Extremity Prosthetic Sockets" (3), and in 1985 on "Computer-Aided Analysis of Below-Knee Socket Pressure"' (4). Also in 1985, the DVA RR\&DS, together with the National Institute for Handicapped Research and the National Aeronautics and Space Administration (NASA), began jointly funding development, under NASA contract, of a limb shape optical sensor for use with prostheticsorthotics CAD/CAM systems.

By 1986, the BK CASD/CAM development program at the UCL-BC in London, England had progressed sufficiently from the laboratory prototype demonstrated at the 1983 ISPO World Congress, that, in addition to intramural clinical trials at the Roehampton Limb Fitting Center in London, an extramural clinical testing program was begun at centers in Stanmore, England, Stockholm and Goteborg, Sweden, and Frankfurt, West Germany. In 1987, the Prosthetics Research Study (PRS) in Seattle, WA, under sponsorship of the DVA RR\&DS, became actively involved in this program and began fitting UCL-BC-designed CASD/CAM sockets in the United States. The level of success achieved in these trials $(5,6)$ was felt to be advanced enough to warrant a more extensive, carefully controlled, clinical testing and evaluation program. Under the leadership of Dr. Ernest M. Burgess, researchers from the PRS; the Northwestern University Prosthetics Research Laboratory and the VA Lakeside Medical Center (NU-VALMC) in Chicago, IL; and the New York Department of Veterans Affairs Medical Center, and New York University Medical Center (NY DVAMC-NYUMC) in New York, NY, began formulation of plans for a collaborative, BK CASD/CAM clinical testing and evaluation program. The proposed program received a high priority in DVA RR\&DS peer review, and the services of Dr. R.M. Davies, Director of the Bioengineering Center at the University College London, were obtained to assist in the initiation of this program. Prototypes of the UCL-BC BK CASD/CAM system were ordered for the three Automated Fabrication of Mobility Aids (AFMA) Centers, and testing and evaluation under the DVA National AFMA Program began October 1, 1988.

The UCL-BC system was selected as the principal CASD/CAM system for testing and evaluation under the AFMA Program, because it was the most complete prosthetics CASD/CAM system available and because initial test results for this system evidenced the highest level of success (7). Limited testing of the UBC-MERU Canfit CASD System was also proposed. These systems were the only prosthetics CASD/CAM systems beyond the research stage at the time that the DVA National AFMA Program was initiated. In accordance with the AFMA Program goal of furthering knowledge and interest in CAD/CAM technologies in prosthetics and orthotics in the United States, PRS hosted an international workshop on CAD/CAM in prosthetics and orthotics in June 1988, under the joint sponsorship of ISPO, the DVA RR\&DS, and the American Academy of Orthotists and Prosthetists (AAOP). The participants in this workshop affirmed the need for a coordinated, carefully controlled, clinical study to test and evaluate objectively 
the capabilities, limitations, applicability, and efficacy of prosthetics and orthotics CAD/CAM techniques and equipment, and to define the areas where further research and development were needed (8).

\section{OBJECTIVES/SCOPE}

The DVA RR\&DS National AFMA Program was formulated to address the key issues identified by the prosthetics researchers, practitioners, and rehabilitation health care experts at the DVA Prosthetics-Orthotics CAD/CAM Workshop in 1985 and the ISPO-DVA-AAOP workshop in 1988. Specifically, the objectives of the National AFMA Program were: $a$ ) to provide a limited introduction of CAD/CAM technologies to prosthetists, physicians, therapists, and other rehabilitation health care professionals throughout the United States; $b$ ) to evaluate the feasibility of utilizing CAD/CAM technologies in clinical prosthetics settings in the United States; $c$ ) to developmentally test and evaluate the UCL-BC and the UBC-MERU BK CASD/CAM systems to determine their capabilities, limitations, applicability, and efficacy; and, $d$ ) to obtain quantitative physiological, biomechanical, prosthetics, and engineering data for use in the refinement of these systems and for use in development of new, enhanced, more efficacious, and more expedient CAD/CAM systems and techniques.

To accomplish these objectives, a four-part program was devised. The first part was a clinical outreach to rehabilitation institutions and prosthetics facilities across the United States. It was felt that an outreach program similar to the UCL-BC extramural testing program would provide an introduction to prosthetics $\mathrm{CAD} / \mathrm{CAM}$ technologies for a broad range of prosthetists, physicians, therapists, and rehabilitation health care professionals by enabling them to participate with an AFMA Center in the design and manufacture of CASD/CAM sockets and prostheses for one or more of their patients. It also was felt that such a program would test the feasibility of utilizing CAD/CAM technologies in ciinical prosthetics settings and demonstrate the efficiency and productivity achievable with prosthetics CAD/CAM technologies. The second part involved developmental testing of the CASD/CAM equipment and software by the three AFMA Centers. Under this part of the program, the AFMA researchers conducted laboratory tests to determine the given systems' physical capacities, precision, accuracy, repeatability, speed, durability, and maintenance requirements. The third part of the AFMA Program involved comprehensive, carefully controlled, clinical testing of the CASD/CAM systems by the AFMA researchers to evaluate their clinical capabilities and limitations, their efficacy, and their productivity. The fourth part consisted of compilation and analysis of the physiological, biomechanical, prosthetics, and engineering data collected during the other phases of the study for determination of refinements needed in the CASD/CAM systems tested and for development of specifications for new, enhanced, more efficacious, and more expedient CASD/CAM systems and techniques.

\section{METHODS}

Because of its role as lead center in the DVA RR\&DS National AFMA Program, and its prior involvement in the UCL-BC extramural clinical testing program, PRS obtained laboratory CASD/ CAM prototype equipment on loan from UCL-BC until production prototypes could be procured. This enabled PRS to begin the outreach phase of the AFMA Program in October 1988. There were no requirements placed on prosthetists or institutions/ facilities for participation in the outreach program, other than that they be competent in the clinical practice of prosthetics, be able to provide one or more patients meeting the AFMA patient protocol, have sufficient interest in the program to collect the experimental data requested, and conscientiously fit and provide accurate feedback regarding the AFMA CASD/CAM sockets. Under the outreach program, the participating prosthetists selected subjects from their patient populations for CASD/CAM socket design, fitting, and evaluation that: (a) were unilateral below-knee amputees; $(b)$ were 18 years of age or older, capable and willing to give their informed consent; $(c)$ were acceptable for prescription of a patellar tendon bearing (PTB) prosthesis; and, $(d)$ had been fit with and worn a BK prosthesis without unusual problems for a period of at least 1 year prior to entry into the program. Preference was given to United States veterans, with all other subjects treated on a first-come, first-served basis. 
Under the outreach program, the participating prosthetists sent PRS undistorted plaster wrap casts of their patients' residual limbs, together with prescribed measurements, socket design requirements, and patient medical, physiological, biomechanical, and prosthetics background information. The PRS AFMA researchers, in turn, digitized the casts and designed and manufactured CASD/CAM sockets for fitting and evaluation by the participating prosthetists and clinic teams. If, on fitting, modifications in a patient's socket were deemed necessary by the participating prosthetists and clinic teams, the locations and magnitudes of the required modifications were noted on an AFMA evaluation form, and the forms were returned to PRS. New sockets were then designed and manufactured by the AFMA researchers and returned to the participating prosthetists. The prosthetists were instructed not to perform any modifications on the CASD sockets, thereby ensuring that all modifications were performed using the CASD system and, hence, were entered into the computerized records of each patient and into the AFMA database. This iterative process was repeated until either a successful fit was obtained or until six unsuccessful fittings had been attempted. When a successful fitting was achieved, a definitive CASD prosthesis was fabricated and delivered to the patient, who was then asked to wear the prosthesis for at least 1 month. The NU-VALMC and NY DVAMC-NYUMC AFMA Centers conducted similar regional outreach programs on receiving, installing, and debugging their CASD/CAM software and equipment.

The second part of the Program involved developmental, laboratory testing of the CASD/ CAM equipment and software to determine the systems' physical capacities, precision, accuracy, repeatability, speed, durability, and maintenance requirements. To test the performance of the CASD/CAM systems and their respective component parts, the AFMA Centers devised a series of experiments. To test system input, several standardized forms with varying geometry were utilized (i.e., sextahedra, conical frustra, and cascaded cylindrical sections with linearly increasing radii). The accuracy, precision, and repeatability with which the dimensions of these calibrated test forms (and the locations and sizes of specified perturbations on their surfaces) were capable of being determined were tested. The marginal repeatability, limited accuracy, and low precision encountered in the original UCL-BC carver-based digitization technique prompted the PRS researchers to develop an electromechanical digitizer for residual limb cast digitization and CASD system input. The PRS and NU-VALMC researchers utilized PRS-designed digitizers throughout the AFMA Program. The NY DVAMC-NYUMC researchers utilized a digitizer they designed that incorporated the same components and was comparable to the PRS digitizer. To evaluate the CASD software, each function was performed at a series of values over its permissible parametric range. The results were recorded and compared with the ideal output. In addition, over the duration of the AFMA Program, log books were kept regarding the type and frequency of occurrence of "bugs" and failures. Test results, together with suggestions for improvements, were periodically compiled and given to the CASD/CAM system developers.

Geometric forms similar to those used to test and calibrate the electromechanical digitizers were mathematically generated, transformed into $\mathrm{CNC}$ code, carved, and measured, and the results compared with the ideal mathematical shapes. Results from a series of these trials were then compiled and averaged to provide measures of the accuracy, precision, and repeatability achievable by the UCL-BC Carve Shape CNC carvers. In addition, tests were performed in which a series of carefully calculated perturbations were introduced on the surfaces of the geometric test shapes. The accuracy, precision, and repeatability with which these shapes and perturbations could be carved were then measured. Similarly, calibrated, positive, geometric models were used to evaluate the accuracy and repeatability with which negative models could be manufactured with the UCL-BC Rapid Shape thermoforming machines. Log books were kept to document the type and frequency of occurrence of errors, malfunctions, and maintenance required by the CAM equipment. The results of these tests, together with suggestions for improvements, were periodically compiled and given to the CASD/CAM system developers.

To measure overall system performance, test shapes with calibrated perturbations were digitized. Modifications at specified locations and of specified geometries were designed into the resulting digitized models. Positive molds of these models were then 


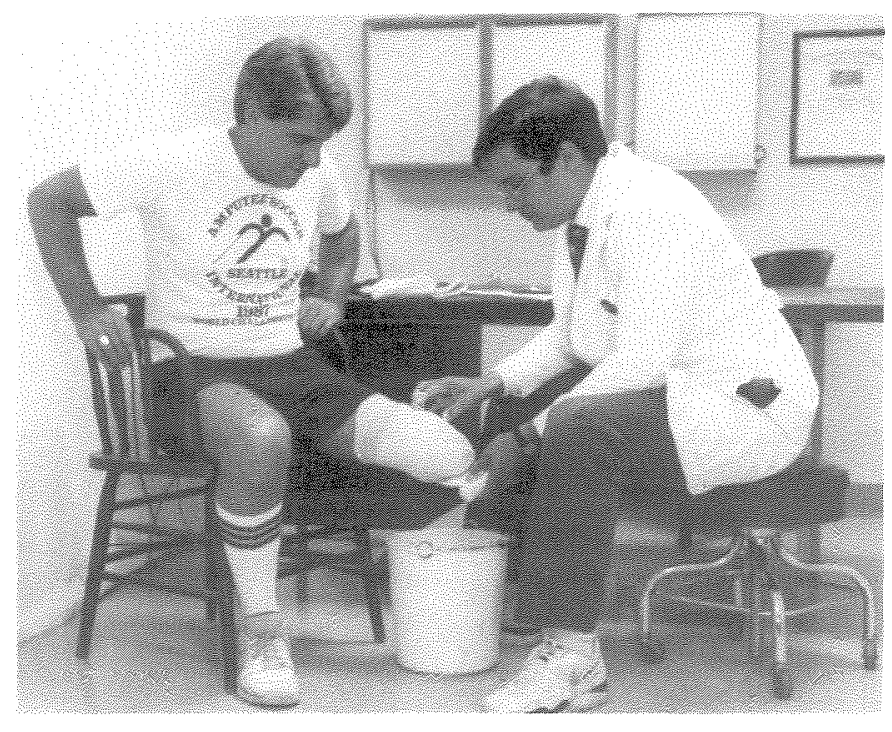

Figure 1 .

Patient evaluation and residual limb characterization through undistorted plaster wrap cast with preservation of location of mid-patellar tendon, for UCL-BC CASD system input.

carved, and negative molds were thermoformed over them. The dimensions, locations, and geometries of the plastic molds that resulted were then compared with the original CASD/CAM system input models as a measure of the overall system accuracy, precision, and repeatability.

In the third part of the AFMA Program, comprehensive, carefully controlled, clinical tests of the CASD/CAM systems were performed. A representative range of $B K$ amputees was selected in accordance with the AFMA Program protocol previously described. Comprehensive medical, physiological, biomechanical, and prosthetics histories, together with prescribed biomechanical and prosthetics measurements, were taken on each subject. Undistorted plaster wrap casts of the residual limbs of the subjects were then taken (Figure 1). The resulting casts were placed in the electromechanical digitizers and aligned relative to the center of mass and mid-patellar tendon of the residual limbs (Figure 2). The casts were digitized, sampling in $10^{\circ}$ angular increments around each cross-section and $6.35 \mathrm{~mm}$ incrementally along the length of each residual limb cast. The resulting digitized measurement files were subsequently input into the UCL-BC Computer Shape CASD System (Figure 3). A socket design template was selected, and an initial socket

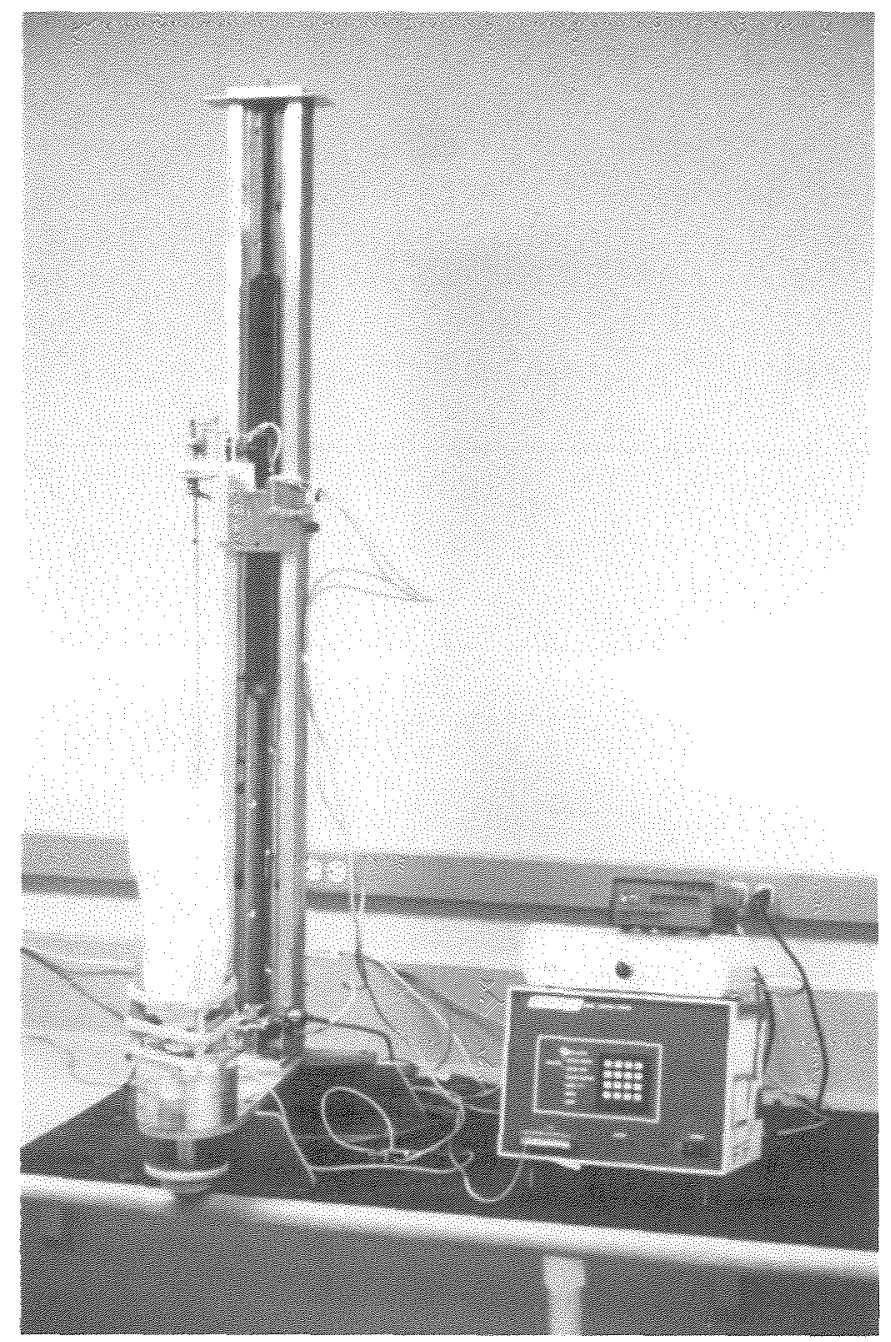

Tigure 2a.

Electromechanical digitizer developed by the Prosthetics Research Study AFMA researchers for digitization of amputees" residual limb casts and sockets for CASD system input.

model was derived by automatically superimposing the template on the digitized residual limb measurements of the patient (Figure 4). A definitive socket model was then obtained by adjusting the template modification region foci magnitudes as required (Figure 5), and by appropriately modifying respective socket node radii to accommodate individual patient anomalies, such as bone spurs, adherent scar tissue, neuromas, cysts, etc.

In the UBC-MERU Canfit CASD System, instead of digitized measurements from residual limb casts, specialized caliper measurements of patients' residual limb length, condylar diameter, tibial angulation, and cross-sectional circumferences 


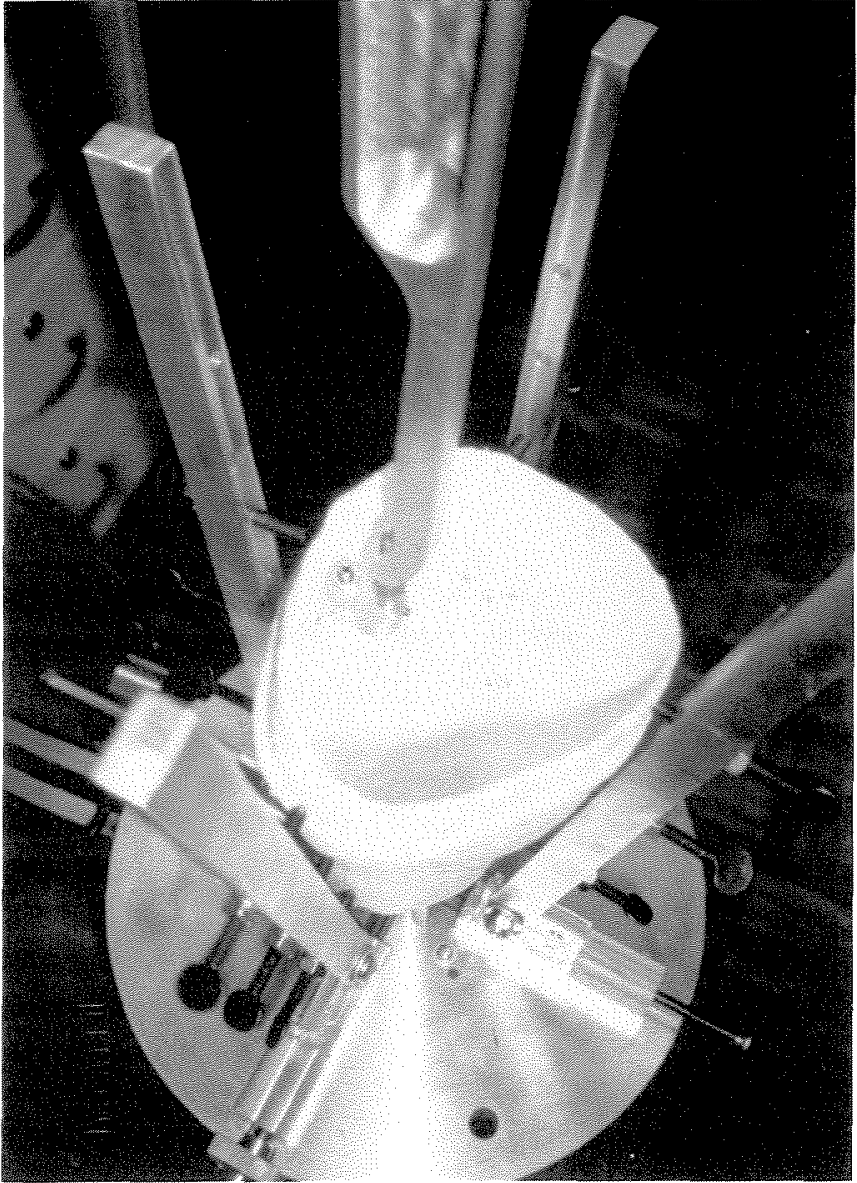

Tigure 2b.

Proximal view of electromechanical digitizer. Shows the digitizer follower arm measuring cross-sectional radii in an undistorted cast of an AFMA test subject's residual limb for UCL-BC CASD system input.

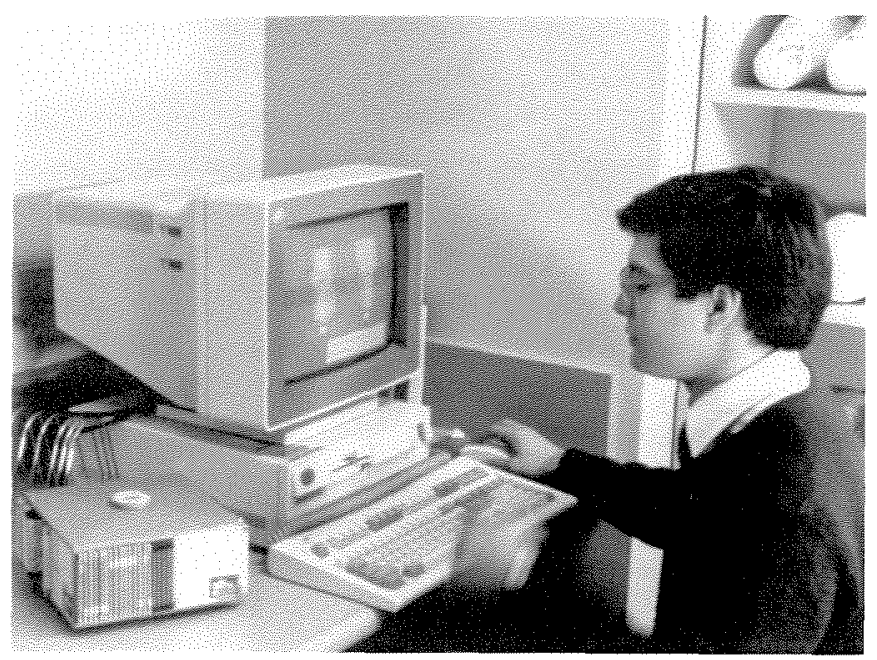

Figure 3.

UCL-BC Computer Shape CASD System socket design for an AFMA test subject.

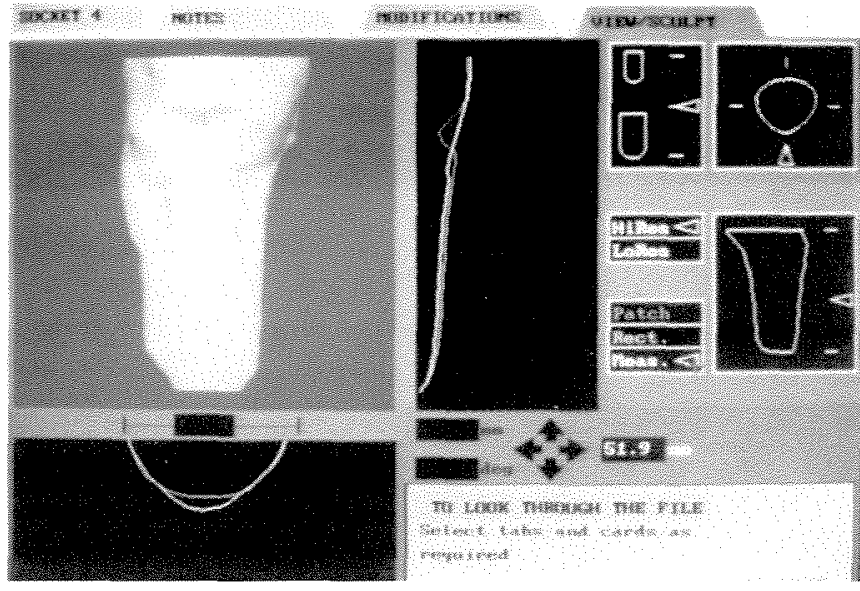

Figure 4.

UCL-BC Computer Shape display for CASD socket design. Shaded surface and cross-sectional contours of the CASD socket and digitized residual limb measurements are displayed for visualization of geometric relationships and contour rectification by socket node radii modification.

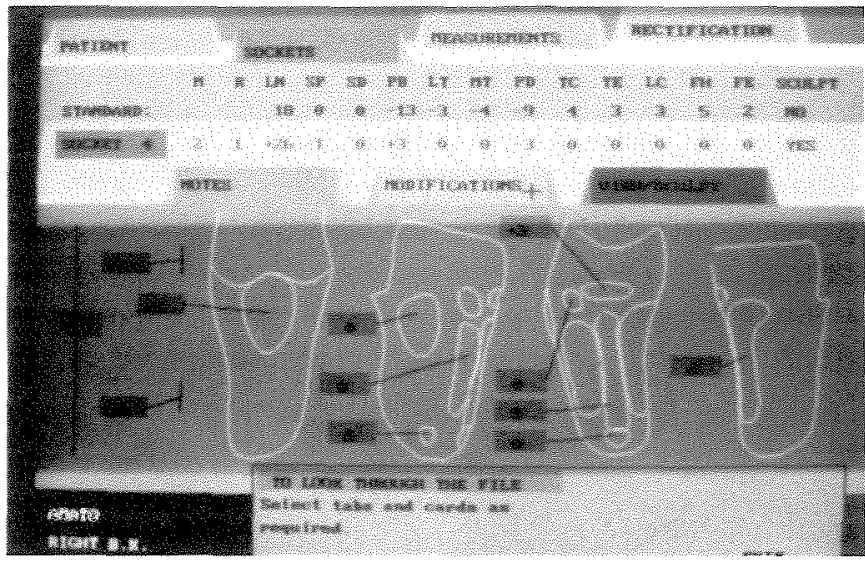

Figure 5.

UCL-BC Computer Shape socket design template. Four orthogonal projections of the CASD socket model are displayed, showing the template modification region locations and the "standard" or default magnitudes of their foci. The foci magnitudes may be interactively modified by the prosthetist through selection of a region and incrementally adjusting the focus magnitude displayed.

at $2.5-\mathrm{cm}$ increments along the length of their residual limbs were taken and input into the CASD system (Figure 6). An initial socket model was obtained from these measurements by automatically scaling and matching, as best as possible, a set of predefined anthropomorphic socket shapes stored in 


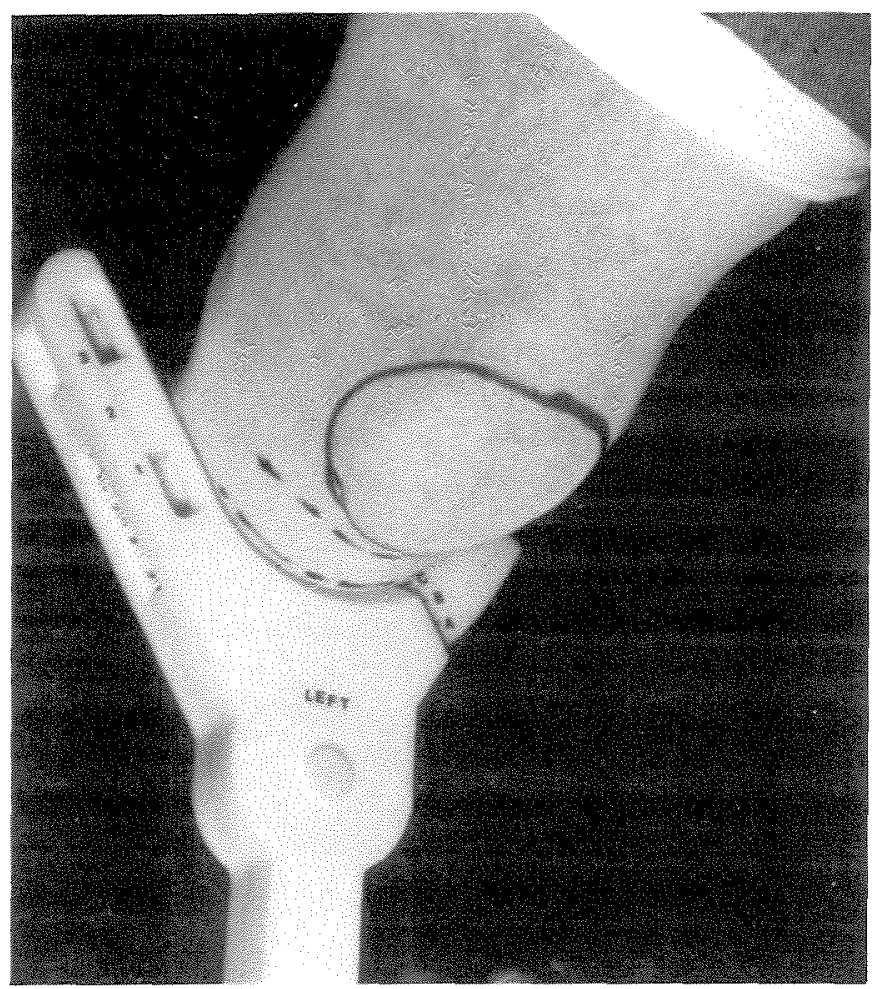

Figure 6.

Patient evaluation and residual limb characterization with the biflanged, tibial angulation/circumferential measurement caliper for UBC-MERU Canfit CASD System input.

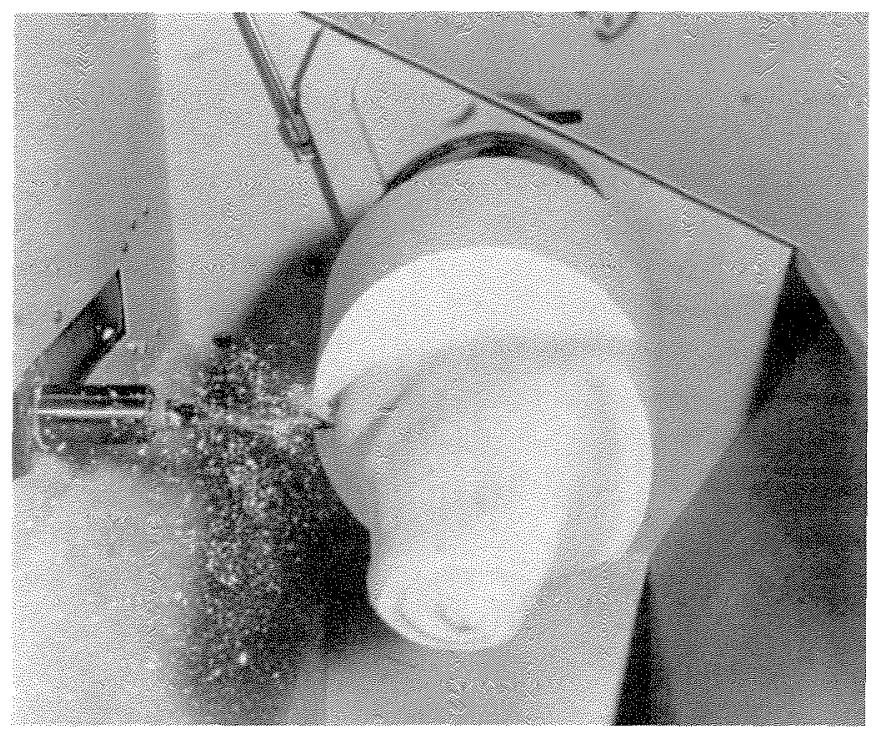

Figure 7 .

UCL-BC Carve Shape CNC milling machine carving a positive CASD socket model for an AFMA test subject from a plaster-corn starch blank.

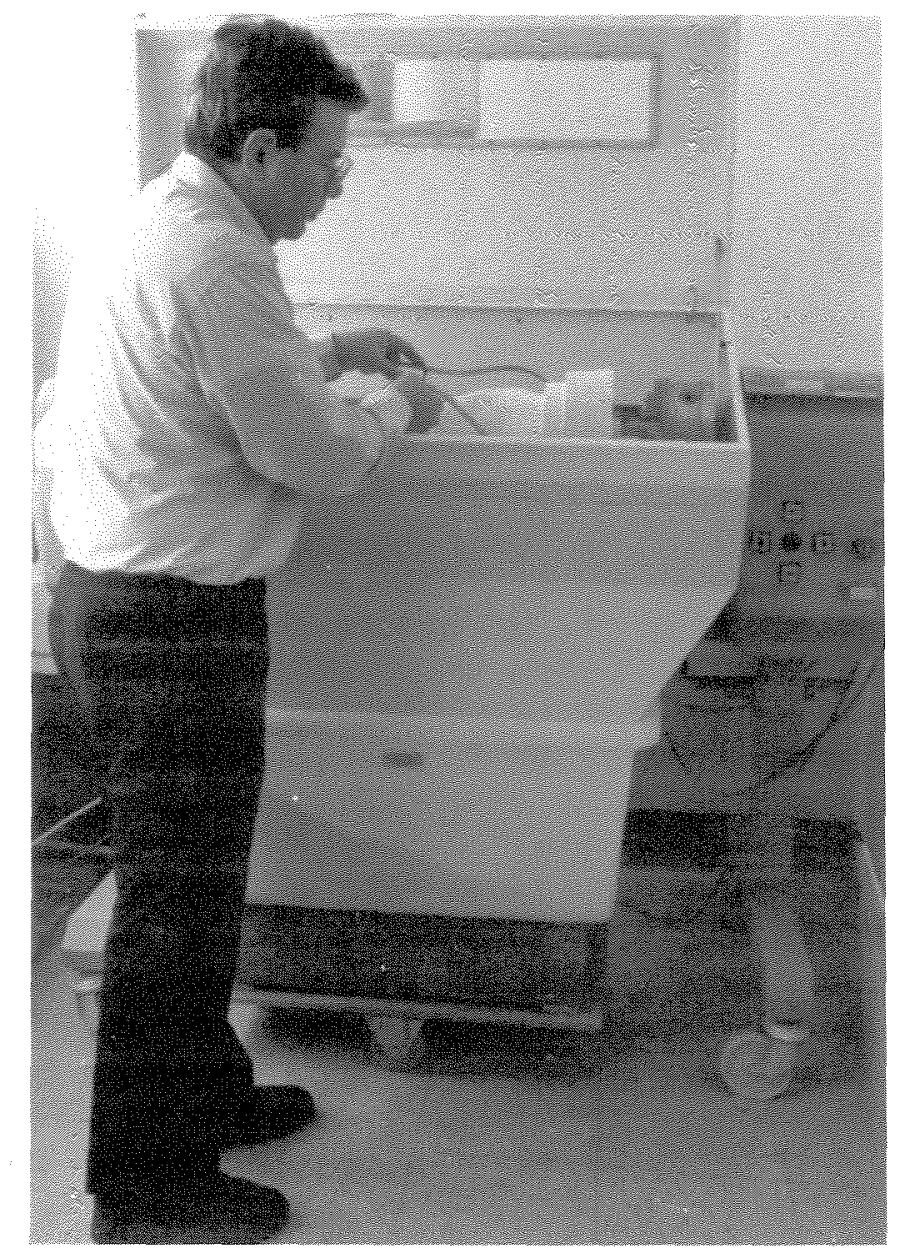

Figure 7b.

Measurement of the calibration "check ring" at the proximal end of a positive model of a CASD socket for verification of the $\mathrm{UCL}-\mathrm{BC}$ Carve Shape milling machine's accuracy.

the computer. A definitive model was obtained from the initial anthropomorphic socket model by identifying and creating any additional modification regions needed to rectify areas of the socket model poorly matched to the residual limb or areas requiring custom modification for individual patient anomalies, such as bone spurs, scar tissue, neuromas, cysts, etc.

When definitive socket models were achieved, the resulting socket design files were sent to the UCL-BC Carve Shape CNC milling machine for carving (Figure 7). When carving was completed, the positive plaster socket models were removed and placed in the UCL-BC Rapid Shape thermoforming machine, where thermoplastic preforms were heated to glass temperature, and automatically pulled down 
and vacuum-formed over the positive plaster models (Figure 8). The plastic was then allowed to cool, the plaster broken out, and the negative socket models appropriately trimmed. The resulting sockets were then fit and evaluated on the test subjects (Figure 9 and Figure 10). If any modifications in the CASD/CAM sockets were deemed necessary, the locations and magnitudes were recorded on the sockets and in an AFMA fitting log book (Figure 11). All modifications were subsequently instituted with the CASD computer. Redesigned CASD sockets were then manufactured and refit and reevaluated on the test subjects. This iterative process was repeated, until either a successful fit was obtained, or until six unsuccessful fittings had been attempted. When successful fittings were achieved, definitive prostheses incorporating the successful CASD/CAM sockets were fabricated and delivered to the subjects (Figure 12). Each subject was carefully followed and reevaluated after 1 month's usage of the CASD/ CAM prosthesis.

The fourth part of the Program consisted of compilation and analysis of the physiological, biomechanical, prosthetics, and engineering data collected during the AFMA Program. The AFMA data were compiled in a computerized relational database and analyzed for trends in CASD/CAM hardware and software problems; correlations between categories of patient physiological, biomechanical, and prosthetics characteristics; and ease/ difficulty in CASD/CAM socket design and fitting.

\section{RESULTS}

\section{AFMA Outreach Program results}

The AFMA Outreach Program, begun in October 1988, introduced prosthetics CASD/CAM technologies to 66 prosthetists, 198 patients, and associated rehabilitation health care staffs in 44 institutions and prosthetics facilities across the United States. In the outreach program conducted by PRS, 49 prosthetists and 118 patients in 32 institutions and prosthetics facilities actively participated. In the regional outreach program conducted by NU-VALMC, 10 prosthetists and 38 patients from 7 prosthetics facilities in the mid-United States actively participated. In the outreach program conducted by the NY DVAMC-NYUMC, 7 prosthetists and 42 patients at 5 institutions in the Eastern

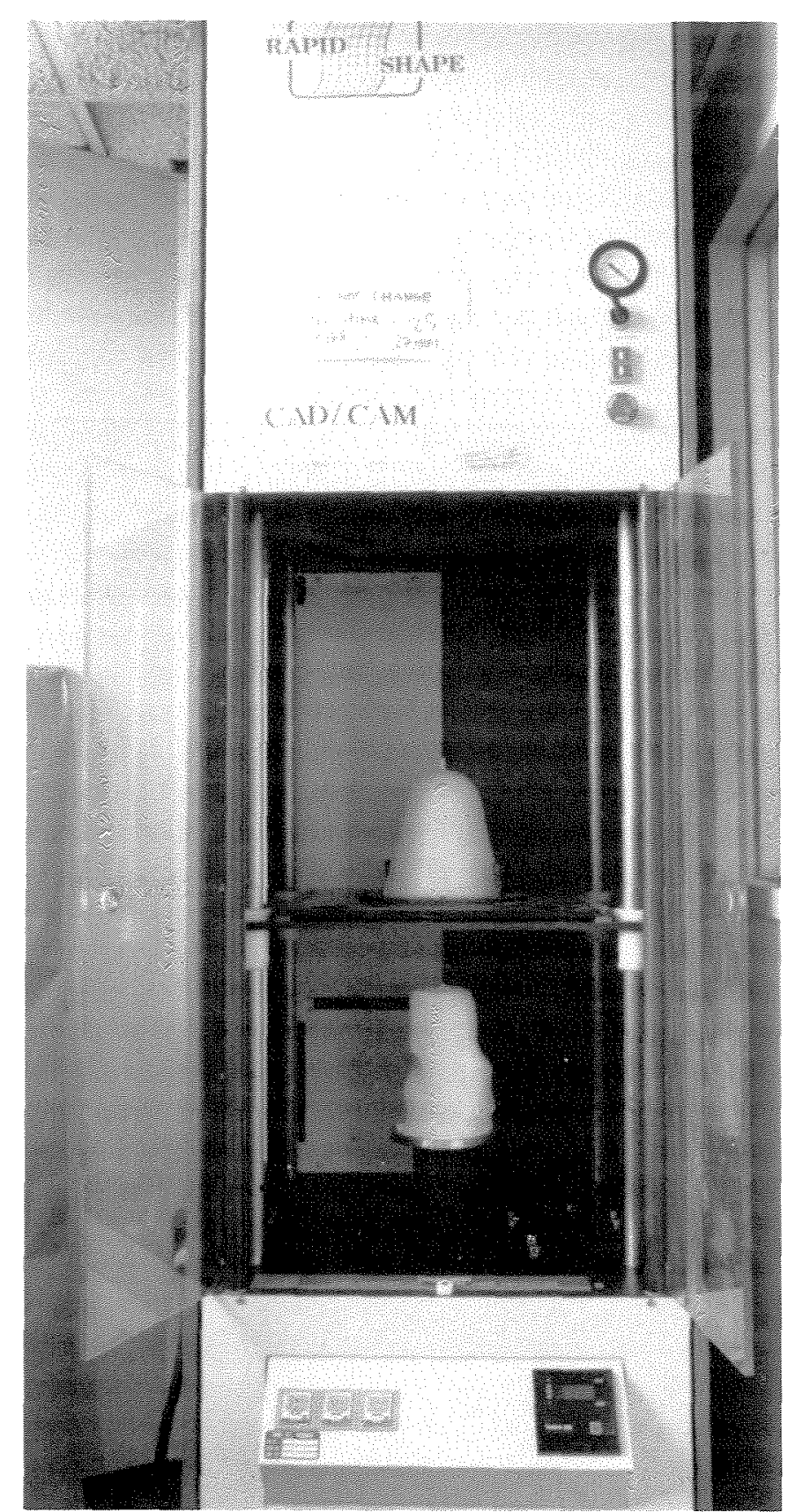

Figure 8.

Manufacture of a negative socket model with the UCL-BC Rapid Shape thermoformer. The plastic preform shown above the positive socket model is heated to glass temperature in the oven in the top of the Rapid Shape, and then automatically pulled down over the positive CASD socket model and vacuum formed around it.

United States were actively involved. The $281 \mathrm{CASD}$ sockets designed and manufactured under the outreach programs at the three AFMA Centers were produced by the Centers' research staffs in addition 


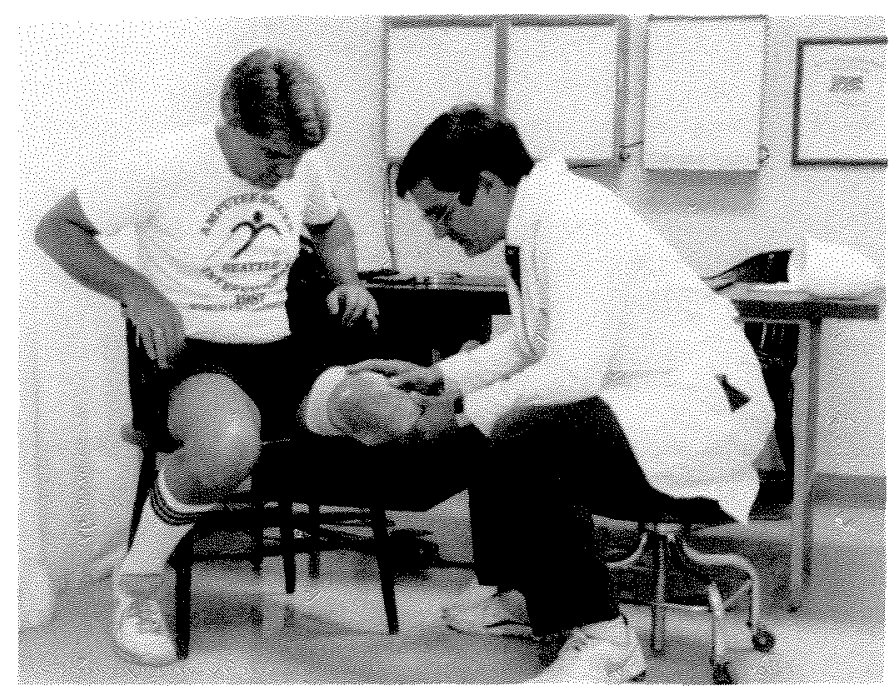

Figure 9.

Static evaluation of the accuracy and quality of fit of a CASD/CAM check socket on an AFMA test subject.

to their other full-time research duties. Even under these circumstances, the design and fabrication of the outreach program CASD sockets was accomplished with an average turnaround time of 2.6 working days, with 46 percent being designed, fabricated, and shipped back to the respective participating institutions/facilities in 1 day or less. These results demonstrated the significant distributed manufacturing capabilities and productivities achievable with prosthetics $\mathrm{CAD} / \mathrm{CAM}$ technologies. As CAD/CAM systems are further refined and enhanced, even greater efficiencies and productivities should be achievable.

\section{Laboratory testing and evaluation results}

Overall, the UCL-BC CASD/CAM system proved to be relatively easy to use. It was found to have a straightforward, convenient user interface. The common experience at each of the AFMA Centers was that the UCL-BC CASD/CAM system required approximately 1 to 2 days to install (assuming a priori site preparation with allocation of appropriate space, and installation of requisite electrical wiring). Similarly, the UBC-MERU CASD system also had a relatively easy-to-use and straightforward graphical user interface. The time required for training in the use of the systems was found to depend on the prosthetists' backgrounds. Those with technical backgrounds and prior computer

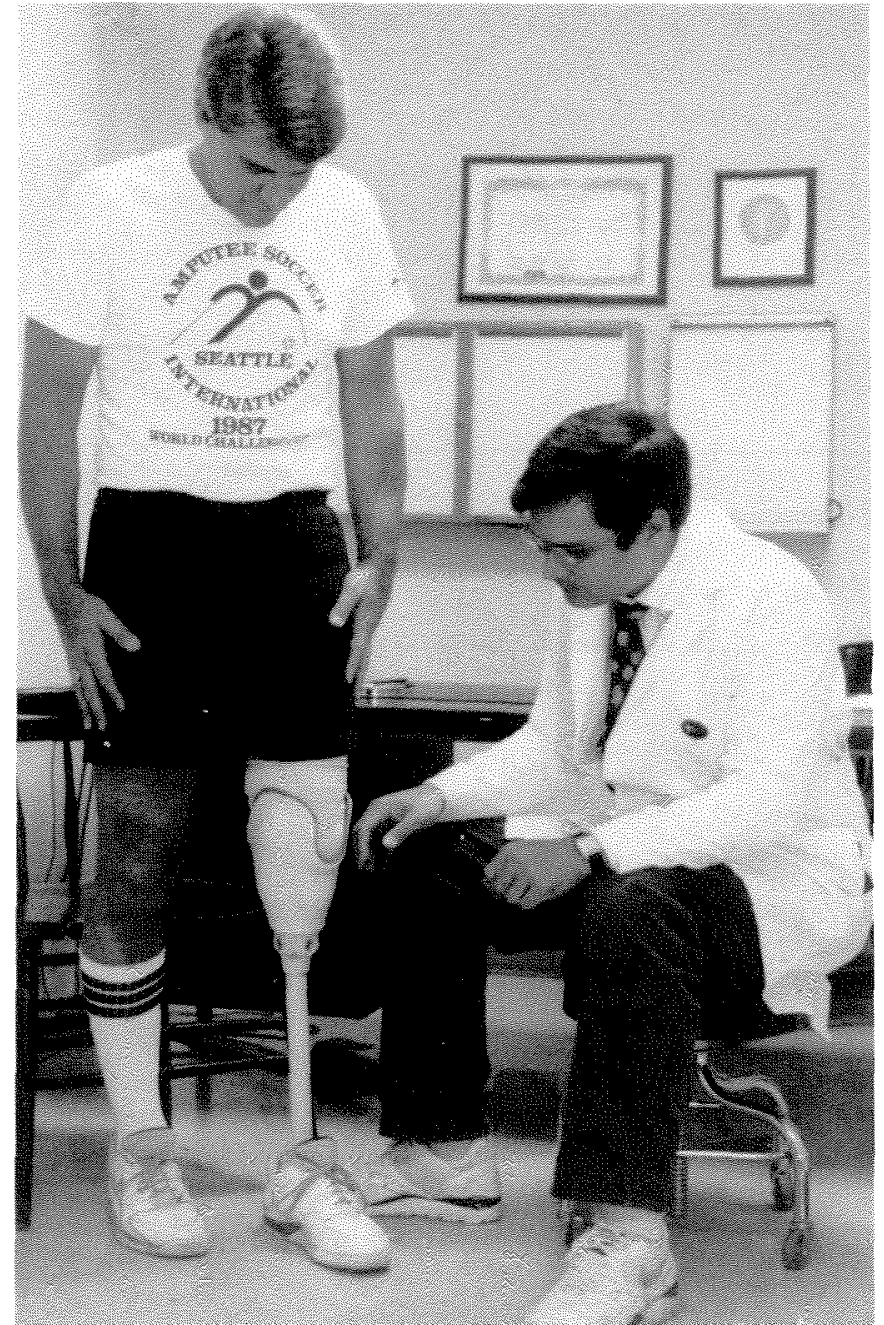

Figure 10.

Dynamic evaluation of the accuracy and quality of fit of a CASD/CAM socket on an AFMA test subject.

experience required approximately 1 day to learn how to use the systems. Individuals with little or no technical background or prior computer experience took up to 4 days to learn how to use the systems. Design, manufacture, and fitting of sockets for approximately four amputees of varying residual limb shapes, sizes, biomechanical characteristics, etc. was found to be required before a reasonable degree of proficiency and a reasonably comprehensive knowledge of the systems were obtained.

Laboratory testing of the CASD/CAM equipment and software was carried out over the duration of the AFMA Program. A significant portion of the 
work in this phase of the program proved to be problem diagnosis and resolution, with developer feedback. This led to an evolution of the CASD/ CAM hardware and software over the course of the AFMA Program. Problems were discovered and diagnosed, suggestions for improvements made, and upgrades were obtained from the developers remedying the problems and incorporating improvements. Changes in the hardware and software were permitted during the AFMA Program, because it was recognized that the given CASD/CAM systems were developmental, and thus required further refinement. It was felt that restriction of testing and evaluation to the systems as originally obtained would be counterproductive, yielding results on rapidly outdated components. During the first year of the AFMA Program, five hardware and four software upgrades were made to the UCL-BC CASD/CAM systems. During the second year, no additional changes were incorporated.

Inaccuracies and limited repeatability were encountered with the cast digitization technique used in the UCL-BC system. The original UCL-BC

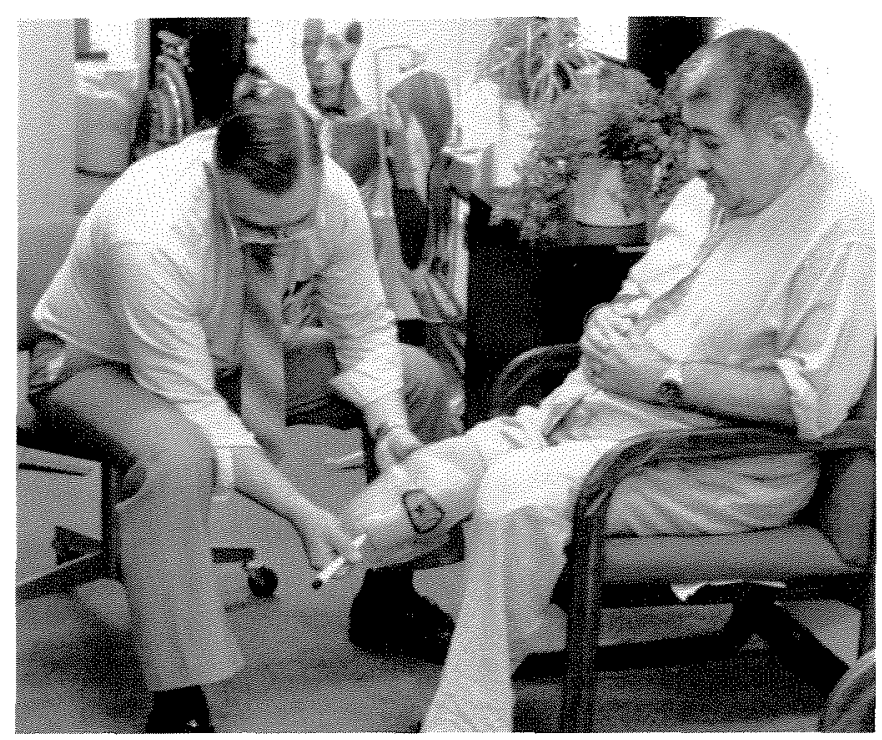

\section{Figure 11.}

Evaluation of the accuracy and quality of fit of a CASD/CAM check socket on an AFMA test subject. The location and magnitude of all modifications in the CASD socket deemed necessary by the AFMA prosthetists during fitting and evaluation, were recorded on the check socket and in an AFMA Fitting Logbook for subsequent CASD system "redesign" and compilation in the AFMA computer database. digitizer consisted of a simple follower arm-potentiometer attached to the Rapid Shape carver for helical, "on-the-fly" digitization of residual limb casts of the patients. This technique proved to be highly dependent on the rigidity and orientation achieved in mounting the casts in the carver, the spatial location and relative severity of cast surface anomalies, and the amount of cast axial/radial taper. To eliminate a number of these problems, to reduce sensitivity to others, and to increase productivity by enabling simultaneous cast digitization and socket carving, the PRS researchers developed a

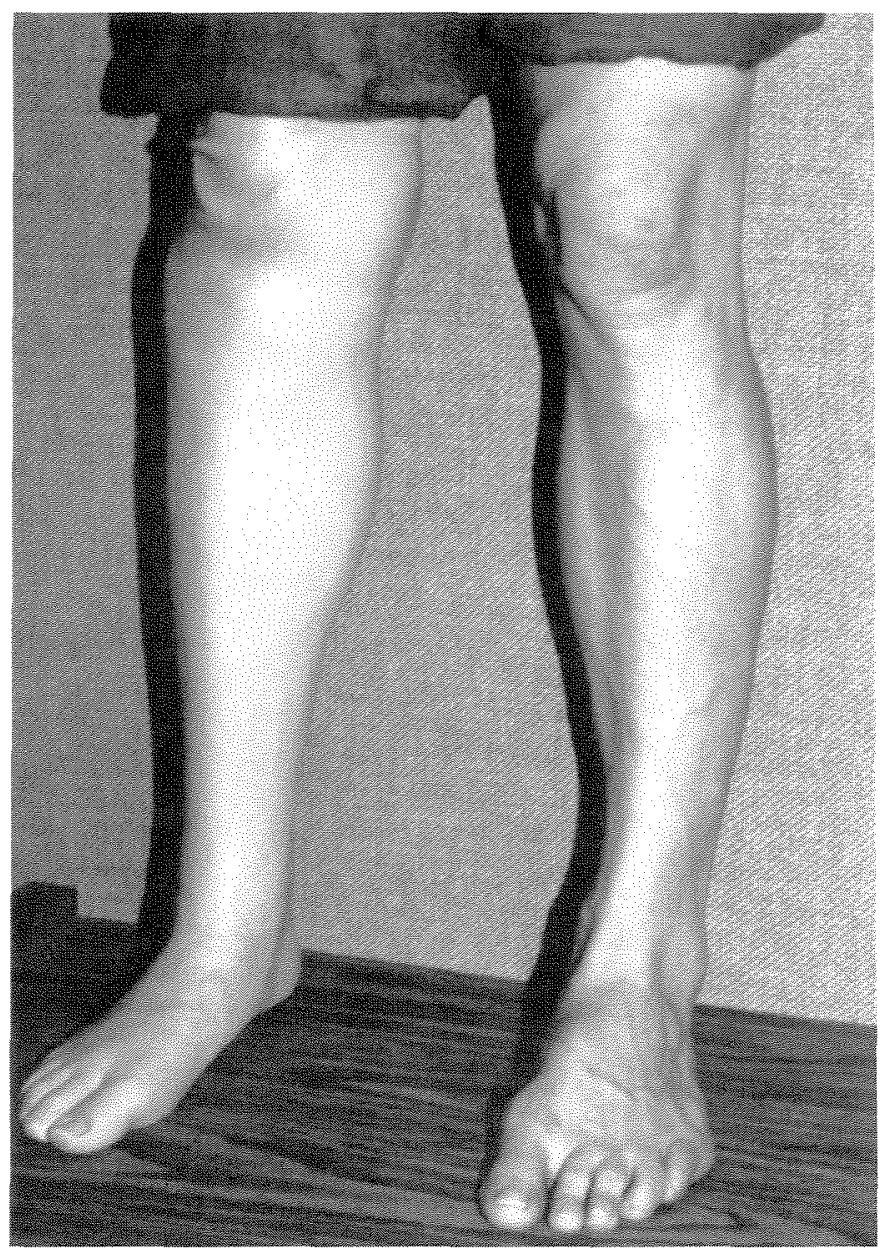

Figure 12.

Definitive CASD/CAM prosthesis delivered to an AFMA test subject for testing and evaluation. When successful CASD socket designs were achieved, definitive CASD/CAM prostheses were manufactured and delivered to the test subjects for normal, daily use for a period of at least 1 month, after which they were asked to return for reevaluation. 
Journal of Rehabilitation Research and Development Vol. 29 No. 4 Fall 1992

computer-controlled, electromechanical digitizer. The PRS digitizer incorporated a computer-controlled, stepper-motor-driven, axial translation unislide and rotational table, coupled with electrooptical transducers for position measurement. This design enabled cast digitizations to accuracies within $\pm 0.10^{\circ}$ rotationally, $\pm 0.0254 \mathrm{~mm}$ translationally, and $\pm 0.150 \mathrm{~mm}$ radially. Schematics and a component list of the PRS digitizer were made available to the UCL-BC CASD/CAM system developers.

A second problem arose because of differences between electrical current and voltage in England and the U.S. Both PRS and NU-VALMC experienced difficulties with their UCL-BC carvers and ended up rewiring them, replacing the transformers and motors. Appropriate design changes were made, and the UCL-BC production prototype carvers subsequently received by PRS and the NY DVAMC-NYUMC AFMA Centers were capable of accepting either European $220 \mathrm{~V} 50 \mathrm{~Hz}$ or U.S. 110 V $60 \mathrm{~Hz}$ electric current. Subsequent testing of the carver received by the NY DVAMC-NYUMC with a Joyce-Lobel controller, revealed that socket contour changes greater than $\pm 10 \mathrm{~mm}$ radially per $10^{\circ}$ rotational increment could not be carved. Furthermore, radial transitions greater than this caused the controller to lose position, rendering all subsequent contours inaccurate, and hence, the resulting socket to be useless. It was also discovered that carving in one-sixteenth-inch pitch resulted in socket models elongated to twice the correct axial length and one-half the correct diameter. The UCL-BC CASD/CAM developers were informed of these and other problems. The Version 1.11 controller upgrade PROMs and associated circuit wiring changes received in July 1989, enabled socket contours with radial perturbations of up to $\pm 25 \mathrm{~mm}$ per $10^{\circ}$ rotational increment to be accurately carved in one-quarter-inch and one-eighth-inch pitches, as per UCL-BC specification. The errors in the onesixteenth-inch pitch carving mode were not remedied, however. Testing also revealed that the postprocessing performed by the UCL-BC CASD software incorporated a limit permitting only positive radial translations at or near the proximal check ring of the carved model. Sockets with proximal diameters larger than $150 \mathrm{~mm}$ were found intermittently to cause the carver to "hang" in the proximal region, ceasing operation, or to plunge cut, destroying the plaster socket mold. In addition, CASD computer-to-carver controller communications problems were encountered in the NY DVAMC Center's carver. Communication errors causing system lockup, with loss of patient data, were found to occur approximately 30 percent of the time. UCL-BC engineers subsequently incorporated pseudo-handshaking for communication acknowledgment between the CASD computer and the carver controller. This adjustment reduced the frequency of occurrence of the problems to less than 5 percent. Although the computer-carver communications faults were never completely resolved, using the "work-around" of backing-up patient measurement and socket design files before carving prevented the remaining communications faults from causing catastrophic loss of data. Version 1.11 of the CASD software also provided improved toolpath checking and contour smoothing, and corrected errors in the CASD program back-up algorithm.

The UCL-BC CASD system performs socket design by superimposing a modification template on the digitized residual limb measurements of a patient. Two UCL-BC socket design templates were available when AFMA Program testing and evaluation began: the "PTB Cuff Suspension Template" by J. MacCoughlan, and the "PTB Cuff Suspension Template for Fleshy Residual Limbs" by J. Wilkinson. Both templates were tested under the AFMA Program; however, only the PTB template by MacCoughlan was used in the comprehensive, clinical testing performed during the third phase. These templates divide the residual limb into four scalable, axial segments: proximal, mid, distal, and end. Each segment, in turn, contains localized, circumferentially distributed modification regions corresponding to standard PTB modifications, empirically derived over the past four decades and currently used in conventional prosthetics practice: length (LN), circumferential size proximal (SP), circumferential size distal (SD), patellar bar (PB), popliteal depression (PD), lateral (pre-) tibial depression (LT), medial tibial flare (MT), tibial crest (TC), tibial end (TE), lateral tibial condyle (LC), fibular head (FH), and fibular end (FE), as shown in Figure $5(9,10,11,12)$. The magnitudes of the foci of these 12 standard PTB modification regions can be interactively adjusted by the prosthetist. To design a socket, the CASD software automatically scales the selected modification template, axially and circumferentially, to match the dimensions of the 
patient's residual limb digitized measurements. The CASD software then calculates cross-sectional centroids of the residual limb at the mid-patellar tendon (MPT) and, at a cross-section two-thirds distally along the residual limb length, aligns the template with the residual limb MPT point and the longitudinal axis between the calculated cross-sectional centroids, and superimposes the template onto the digitized residual limb model. If further rectifications are deemed necessary, the prosthetist can interactively increase/decrease the relative magnitude of the foci of 1 or more of the template's 12 standard modification regions or utilize the CASD patching mode to adjust the radii at selected nodes of the socket model. Modifications over rectangular regions, defined by the nodes on the major and minor axes of rectangle, can also be interactively introduced by the prosthetist in the patching mode.

Tests of the UCL-BC CASD software revealed it to have inherent limits in the length of residual limbs for which sockets could be designed. Fatal errors, causing termination of the CASD program, were encountered when socket design was attempted for residual limbs shorter than $8.0 \mathrm{~cm}$ or longer than $38.5 \mathrm{~cm}$. It was also noted that sockets designed and carved for short residual limbs, slightly greater than the $8.0 \mathrm{~cm}$ minimal allowable length, contained transverse ridges. This presumably occurred because of difficulties in blending the modification template's overlapping, axial segments. These incongruities were especially noticeable for short, conical residual limbs. Testing also revealed that the order in which modifications were performed in the UCL-BC CASD system were important. This occurred because the standardized PTB template modifications are implemented in the UCL-BC CASD system as linear radial magnitude changes, whereas patching operations are affine, radial transformations. Hence, to preserve contour relationships, patching should be the last task performed during the design process. For example, if straight, smooth patellar bar contours are desired, any regional radial modifications needed should be made using the $\mathrm{UCL}-\mathrm{BC}$ template modification regions, and then existing irregularities smoothed via patching. If the order of these operations is reversed, straight, smooth contours will not be obtained, and further patching will be required.

Testing of the Rapid Shape thermoforming machines revealed problems in the PRS and NY
DVAMC-NYUMC units. NU-VALMC did not report any difficulties with their Rapid Shape thermoformer. The PRS and NY DVAMC Rapid Shape thermoforming platen drive assemblies both became misaligned-the PRS unit from excessive friction between the platen and the guide posts, and the NY DVAMC unit from a pull over a large, asymmetric cast. These situations both caused the platen drive assemblies to jam and, subsequently, to break the drive chain in the PRS unit, and to shear off the attachment pins in the NY DVAMC unit. The AFMA researchers realigned the equipment, lubricated the platen guide posts, and installed a new drive chain and hardened attachment pins, respectively. No further problems were encountered in the PRS Rapid Shape. In the NY DVAMC machine, however, in an unrelated incident, the actuating elements of three of the microswitches controlling the travel of the thermoforming platen were struck by the platen and broken. On disassembly of the unit, it was discovered that the sheetmetal wall of the Rapid Shape on which the microswitches were mounted had been dented, presumably while moving the Rapid Shape to a different room in the laboratory, thus causing misalignment of the switches. The Rapid Shape is designed so that when the thermoforming platen reaches the socket mold pedestal, screw heads on the platen actuate the microswitches mounted on the Rapid Shape wall, opening the motor circuit, thereby limiting further travel of the platen. As the microswitches are directly in the path of the platen, misalignment of the switches can cause them to be struck and broken. When this occurred in the NY DVAMC unit, replacements could not be found in the U.S., so the AFMA investigators had to fabricate new switch actuators and repair the switches themselves. In remounting the microswitches, excessive over-travel of the platen because of motor inertia was discovered. The drive assembly slip clutch friction was increased to prevent this. The over-current limit was also reduced to cause earlier actuation of the platen drive circuit breaker, thus reducing the likelihood of damage to the platen and drive assembly, should over-travel occur. The platen and drive assembly in the NY DVAMC unit became misaligned from asymmetric cast pulls on two subsequent occasions, but no damage was incurred because of the above modifications. Recommendations were made to the UCL-BC CASD/CAM 
developers for design changes in the Rapid Shape to avoid these problems.

The digitizers, computers, and CASD software required no maintenance over the period of the AFMA study. As a precaution, however, the calibration of the digitizers was routinely checked to verify the accuracy of the digitized residual limb measurements input into the UCL-BC CASD system. Because of the pervasive and corrosive plaster dust generated during the CAM carving process, the CASD computer and digitizer had to be isolated from the carver to prevent corrosion and potential problems. Periodic cleaning of the carvers was required to remove the plaster dust that built up on the motors and drive rails and in the controller air filters. In addition, over a period of a year, recalibration of the carver longitudinal and transverse axes was required three times. No additional maintenance was required by the UCL-BC Carve Shape equipment. It was discovered that lubrication of the Rapid Shape thermoformers' platen guide rails was periodically required to prevent excessive friction, and potential misalignment and jamming of the drive assembly, as occurred in the PRS unit. In addition, when damp or wet plaster socket molds were used frequently in the Rapid Shape thermoformers, it was necessary to drain the Rapid Shape vacuum lines. No additional maintenance of the UCL-BC CASD/CAM equipment was found to be necessary during the AFMA Program.

Tests of the UCL-BC CASD/CAM system showed the major factor governing system accuracy was the large sampling interval $\left(10^{\circ}\right.$ angularly and $6.35 \mathrm{~cm}$ axially) used in the digitized residual limb measurement and socket models. The CASD computers had either 16- or 32-bit precision, and the digitizers had 8-bit precision; therefore, the digitized residual limb and socket models used in the CASD computers were accurate to within $\pm 0.10^{\circ}$ rotationally, $\pm 0.0254 \mathrm{~mm}$ axially, and $\pm 0.150 \mathrm{~mm}$ radially. Because of the large sampling increments between digitized measurement points, however, the accuracy with which specific points and regions on a residual limb of a patient could be identified was limited to $\pm 5.0^{\circ}$ rotationally, $\pm 3.175 \mathrm{~mm}$ axially, and $\pm 0.254 \mathrm{~mm}$ radially. The UCL-BC CASD software further constrained radial accuracy by limiting radial changes to $\pm 0.5 \mathrm{~mm}$ increments. The toolpath clearance and socket contour smoothing performed by the CASD postprocessor and carver controller further limited the radial accuracy achievable. Tests of the UCL-BC carvers showed them to have positioning resolutions of $\pm 0.10^{\circ}$ rotationally, $\pm 0.0254 \mathrm{~mm}$ axially, and $\pm 0.0254 \mathrm{~mm}$ radially. Residual limb and socket radial contour changes sharper than $2.5 \mathrm{~mm}$ per degree were not preserved by the carvers. The rotational and axial locations of given contour changes were determined to within the accuracies noted above, but the contour radii that were actually carved were locally smoothed averages because of postprocessing toolpath clearance and smoothing changes. Ball end mill bits smaller than the $18-\mathrm{mm}$ diameter bit used during the AFMA Program would allow preservation of sharper radial contour changes, but they would fail to carve smooth contours as well as the $18-\mathrm{mm}$ mill bits, and would significantly increase the time required to carve sockets. The UCL-BC carvers require approximately 15 to 25 minutes to carve an average positive BK socket model, so any increases in carve time beyond this were felt to be detrimental. Use of $18-\mathrm{mm}$ mill bits, therefore, was felt to be a reasonable compromise.

None of the socket models designed and manufactured during the AFMA Program had severe undercuts, so all contours were well preserved in the sockets manufactured with the Rapid Shape thermoforming equipment. The accuracy achieved with the Rapid Shape equipment was primarily a function of the amount of plastic shrinkage that occurred. On the average, $1 \mathrm{~mm}$ (or less) shrinkage was experienced with the polypropylene copolymer plastic preforms used in the AFMA Program.

\section{Clinical testing and evaluation results}

The third part of the AFMA Program involved comprehensive, carefully controlled clinical testing of the CASD/CAM systems to evaluate their capabilities and limitations, their efficacy, and their productivity. In this part, subjects were carefully selected in accordance with the previously developed AFMA protocol. Comprehensive questionnaires and extensive measurements were taken on each subject, and a computerized relational database compiled containing all pertinent patient medical and physiological conditions, biomechanical characteristics, functional requirements, prosthesis characteristics and usage history, and each subject's rating of the prosthesis with respect to overall performance, fit, comfort, function, cosmesis, and weight. Undis- 
torted plaster-wrap casts of the subjects' residual limbs were taken and the resulting casts digitized. CASD sockets were designed, thermoplastic check sockets were fabricated, and then fit and evaluated on each subject. If, on fitting, modifications in a subject's CASD socket were deemed necessary, the locations and amounts of the required modifications were recorded on the check socket and in an AFMA fitting logbook. The specified modifications were subsequently incorporated in the socket model with the CASD computer, and a new CASD socket was manufactured. Modifications of the check sockets per se by the AFMA prosthetists were not permitted. This ensured that all modifications were instituted using the CASD system, and thus, were recorded in the patients' computer files and in the computerized AFMA database. This iterative process was repeated until a successful fit was obtained, at which time a definitive prosthesis was fabricated and delivered to the subject. Each subject was asked to wear the definitive AFMA prosthesis full-time, performing normal daily activities for a minimal period of 1 month. After 1 month's usage without complications, each subject was asked to return and was thoroughly reevaluated.

A total of 90 subjects were enlisted in the comprehensive clinical trials, 80 males and 10 females. Of these subjects, 49 completed the entire study. The other 41 either dropped out of the study because of death, medical conditions, time constraints, geographic relocation, or failed to have their pre-AFMA and AFMA measurements, questionnaires, and test data completed because of other factors. Of the 49 subjects completing the entire study, 43 were males and 6 were females. As shown in Tables 1a and 1b, 66 percent of the AFMA test subjects were U.S. veterans. Forty-four percent were right amputees and 56 percent were left. The average age of the AFMA test subjects was 55 years, with a standard deviation of \pm 14.5 years and a median of 60 years. Sixty-six percent of the subjects had amputations because of traumatic injuries, 31 percent because of peripheral vascular disease, and 3 percent for other reasons. The average age of the subjects amputated because of trauma was 51 years, with a median age of 52 , a standard deviation of \pm 15.3 years, a minimal age of 24 , and a maximal age of 79. The average age of the subjects amputated because of peripheral vascular disease was 64 years, the median was 65 , with a standard deviation

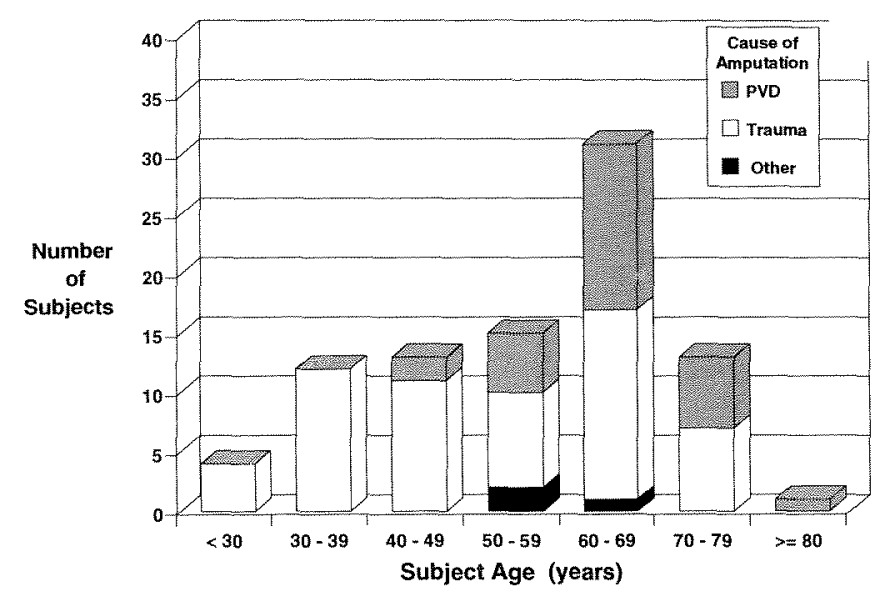

Figure 13.

Number of subjects participating in the AFMA comprehensive clinical testing program categorized by age and cause of amputation.

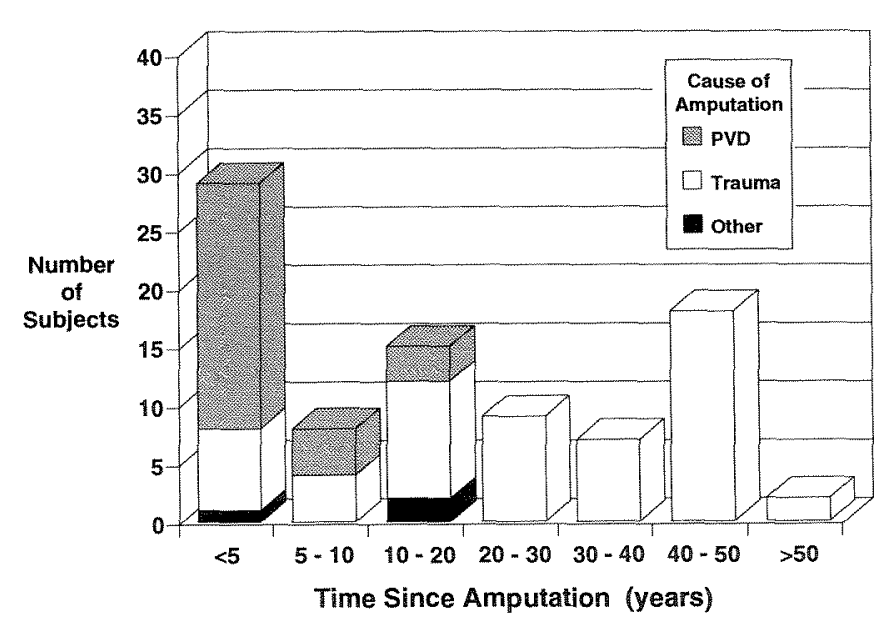

Figure 14.

Number of AFMA test subjects categorized by the number of years since amputation and by cause of amputation.

of \pm 8.8 years, a minimal age of 40 , and a maximal of 84. As seen in Figure 13 and Figure 14, of the test subjects that had amputation in the past 5 years or less, 75 percent had amputation because of peripheral vascular disease (PVD), and of these PVD amputees 75 percent were 60 years of age or older. All but two of the test subjects who were less than 40 years of age were traumatic amputees. The two exceptions had amputations because of cancer. Similarly, all of the subjects who sustained their amputations 15 or more years ago had amputations 
Journal of Rehabilitation Research and Development Vol. 29 No. 4 Fall 1992

Table 1a.

AFMA clinical test subject characteristics.

\begin{tabular}{|c|c|c|}
\hline Characteristic & $\begin{array}{c}\text { Number of } \\
\text { Subjects }\end{array}$ & Percentage \\
\hline \multicolumn{3}{|l|}{ Sex } \\
\hline Male & 80 & 89 \\
\hline Female & 10 & 11 \\
\hline \multicolumn{3}{|l|}{ Race } \\
\hline Caucasian & 64 & 71 \\
\hline Black & 16 & 18 \\
\hline Hispanic & 7 & 8 \\
\hline Undeclared & 3 & 3 \\
\hline \multicolumn{3}{|l|}{ Military Service } \\
\hline U.S. Veteran & 59 & 66 \\
\hline Nonveteran & 31 & 34 \\
\hline \multicolumn{3}{|l|}{ Cause of Amputation } \\
\hline Trauma & 59 & 66 \\
\hline PVD & 28 & 31 \\
\hline Other & 3 & 3 \\
\hline \multicolumn{3}{|l|}{ Side of Amputation } \\
\hline Right & 40 & 44 \\
\hline Left & 50 & 56 \\
\hline \multicolumn{3}{|l|}{ Usage of Ambulation Aids } \\
\hline No Assistive Device & 62 & 69 \\
\hline Cane & 23 & 26 \\
\hline Crutch & 2 & 2 \\
\hline Walker & 1 & 1 \\
\hline \multicolumn{3}{|l|}{ Ambulation Environment } \\
\hline Indoors Only & 3 & 3 \\
\hline Indoors and Stairs & 2 & 2 \\
\hline \multicolumn{3}{|l|}{ Indoors, Outdoors, } \\
\hline No Stairs & 1 & 1 \\
\hline Indoors, Outdoors, Stairs & 3 & 3 \\
\hline \multicolumn{3}{|l|}{ Indoors, Outdoors, Uneven } \\
\hline Ground, No Stairs & 7 & 8 \\
\hline \multicolumn{3}{|l|}{ Indoors, Outdoors, Uneven } \\
\hline Ground, Stairs & 66 & 73 \\
\hline
\end{tabular}

because of traumatic injuries. This is evidenced in the distribution of the number of subjects versus years since amputation in Figure 14, where modes exist corresponding with World War II, the Korean War, and the Vietnam War. Of the AFMA test subjects who had amputations because of trauma or other nonvascular causes, 56 percent reported to be generally in good health, with no major medical complications or associated conditions adversely affecting their functional capabilities and prosthesis usage. In contrast, all of the test subjects amputated because of PVD reported one or more additional medical complications (Figure 15). In spite of this,
Table 1a (Continued).

AFMA clinical test subject characteristics.

\begin{tabular}{|c|c|c|c|}
\hline Characteristic & & $\begin{array}{l}\text { Number of } \\
\text { Subjects }\end{array}$ & Percentage \\
\hline \multicolumn{4}{|l|}{ General Health } \\
\hline Good* & & 35 & 39 \\
\hline Respiratory & & 3 & 3 \\
\hline Arthritis & & 23 & 26 \\
\hline Cardiovascular & & 24 & 27 \\
\hline Peripheral Vascular & & 24 & 27 \\
\hline Diabetes & & 24 & 27 \\
\hline Visual Impairment & & 14 & 16 \\
\hline Balance Impairment & & 21 & 23 \\
\hline \multicolumn{4}{|l|}{ Stump Shape } \\
\hline Bulbous & & 4 & 4 \\
\hline Cylindrical & & 46 & 51 \\
\hline Conical & & 38 & 42 \\
\hline Other & & 2 & 2 \\
\hline \multicolumn{4}{|l|}{ Stump Soft Tissue Amt. } \\
\hline Little & & 22 & 24 \\
\hline Average & & 60 & 67 \\
\hline Considerable & & 8 & 9 \\
\hline \multicolumn{4}{|l|}{ Stump Soft Tissue Type } \\
\hline Very Soft & & 2 & 2 \\
\hline Soft & & 11 & 12 \\
\hline Average & & 68 & 76 \\
\hline Firm & & 9 & 10 \\
\hline \multicolumn{4}{|l|}{ Stump Skin Type } \\
\hline Transparent & & 2 & 2 \\
\hline Thin & & 10 & 11 \\
\hline Normal & & 62 & 69 \\
\hline Thickened/Tough & & 16 & 18 \\
\hline \multicolumn{4}{|l|}{$\begin{array}{l}\text { Stump Distal Tissue } \\
\text { Pressure Tolerance }\end{array}$} \\
\hline \multirow[t]{2}{*}{ None } & 0 & 2 & 2 \\
\hline & 1 & 4 & 5 \\
\hline \multirow{3}{*}{ Moderate } & 2 & 11 & 12 \\
\hline & 3 & 13 & 15 \\
\hline & 4 & 29 & 32 \\
\hline Considerable & 5 & 30 & 34 \\
\hline
\end{tabular}

*Good $=$ no major complications or medical conditions

almost all of the AFMA test subjects were moderately to very active. Seventy percent of the test subjects used no assistive devices to ambulate, 26 percent used canes, 2 percent used forearm crutches, and one subject used a walker. Seventy-three percent of the subjects stated they could walk more than one-quarter of a mile in any kind of community environment (i.e., indoors, outdoors, on stairs, uneven ground, etc.). Twenty-four percent reported 
Table 1b.

AFMA clinical test subject characteristics.

\begin{tabular}{lccccc}
\hline Characteristic & Median & Avg. & SD & Min. & Max. \\
\hline AGE (yrs) & 60 & 55 & 14.5 & 24 & 84 \\
All subjects & 52 & 51 & 15.3 & 24 & 79 \\
$\begin{array}{l}\text { Subjects with amputation due to } \\
\quad \text { trauma }\end{array}$ & & & & & \\
Subjects with amputation due to PVD & 65 & 64 & 8.8 & 40 & 84 \\
WEIGHT (lbs) & 175 & 179 & 38 & 98 & 280 \\
YEARS SINCE AMPUTATION (yrs) & 16.8 & 19.7 & 17.9 & 0.2 & 64.5 \\
STUMP LENGTH (cm) & 14.6 & 14.9 & 4.3 & 8 & 38 \\
& & & & & \\
(\%) of Contralateral limb length) & $29.1 \%$ & $29.5 \%$ & $6.8 \%$ & $13.4 \%$ & $52.2 \%$ \\
AVERAGE DAILY PROSTHESIS & & & & & \\
USAGE (hrs/day) & & & & & \\
$\quad$ Total Daily Usage & 16 & 14.4 & 3.4 & 4 & 21 \\
Sitting & 6 & 6.1 & 2.9 & 1 & 14 \\
$\quad$ Standing & 4 & 3.7 & 2.1 & 0 & 10 \\
$\quad$ Walking & 4 & 4.3 & 2.4 & 0 & 10 \\
Running & 0 & 0.1 & 0.4 & 0 & 2 \\
\hline Avg. = average; SD = standard deviation; Min. = minimum; Max. $=$ maximum & \\
\hline P & & & & & \\
\hline
\end{tabular}

they could walk 500 to 1,300 feet, but were somewhat restricted in their ambulation environment, because of difficulty negotiating either uneven ground, or stairs, or both. Only two subjects reported they could not walk 500 or more feet in their prostheses, and were limited primarily to indoor ambulation without stairs.

On the average, the AFMA test subjects used their prostheses 14.4 hours per day, of which an average of 6.1 hours were spent sitting, 3.7 hours standing, and 4.3 hours walking. Only two younger subjects with traumatic amputation ran, for an average of 0.2 hours per day. For those subjects who used their prostheses approximately 3 to 11 hours per day, there was a relatively proportional increase in each type of activity as their overall prosthesis usage increased, with the exception of running (Figure 16). Above approximately 12 hours total daily usage, sitting was the major activity that increased with prosthesis use. Standing plateaued at around 4 to 4.5 hours per day, and walking plateaued at around 4.5 to 5 hours per day. Further analysis showed that, on the average, the AFMA test subjects with traumatic amputations used their prostheses 15.6 hours per day, of which an average of 6.1 hours were for sitting, 4.3 hours for standing, and 4.8 hours for walking. The test subjects with amputations due to PVD used their prostheses an average of 11.9 hours per day, of which an average of 6.3 hours were for sitting, 2.4 hours for standing, and 3.0 hours for walking. Thus, both groups sat, on the average, approximately an equivalent amount of time. The greater prosthesis usage by the subjects with traumatic amputation corresponded to increased standing and walking. From what is known of the general amputee population in the United States, these statistics are reasonably representative $(13,14,15,16)$.

A slight skewing in the distribution of subjects with respect to residual limb skin type, conditioned on cause of amputation, also occurred. Two subjects with amputations due to PVD had transparent residual limb skin, eight other PVD amputees (29 percent) had thin skin, and the remaining PVD amputees (64 percent) had normal skin. Two subjects with traumatic amputation had thin skin, and the remainder had normal (69 percent) or thickened/tough skin ( 27 percent). The other characteristics affecting residual limb/socket loading and socket fit (e.g., residual limb length, residual limb shape, amount and relative durometer of residual limb soft tissue) all had conditional distributions 
Journal of Rehabilitation Research and Development Vol. 29 No. 4 Fall 1992

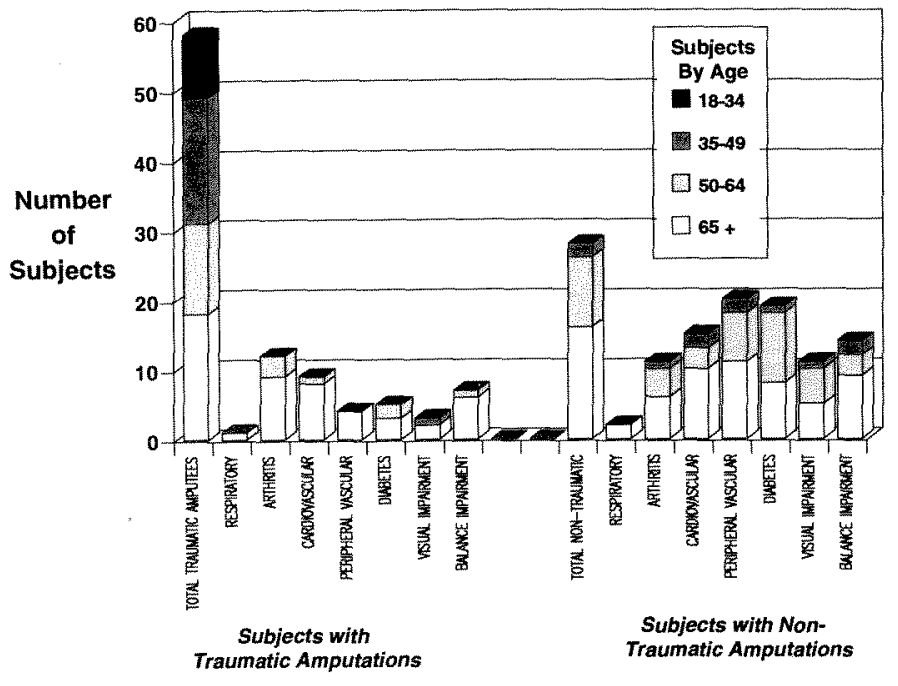

Health \& Medical Conditions of AFMA Subjects

Figure 15.

Number of AFMA test subjects, categorized by age and cause of amputation, with associated medical conditions affecting their prosthesis usage.

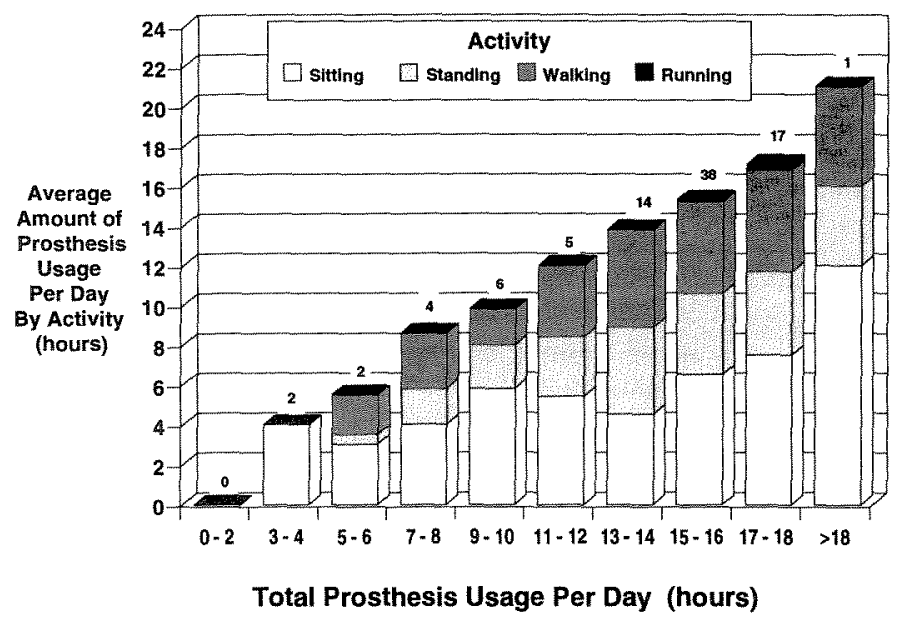

Figure 16.

AFMA test subject average daily prosthesis usage by activity. (The number of subjects using their prostheses on an average daily basis for the designated activities and amounts of time is shown above each column.)

that were approximately the same for the traumatic and the dysvascular groups of test subjects. Similarly, statistically significant differences did not occur in the distributions of subject distal residual limb pressure tolerance, when conditioned on cause of amputation (Figure 17). Both the dysvascular and the traumatic groups evidenced a broad range of

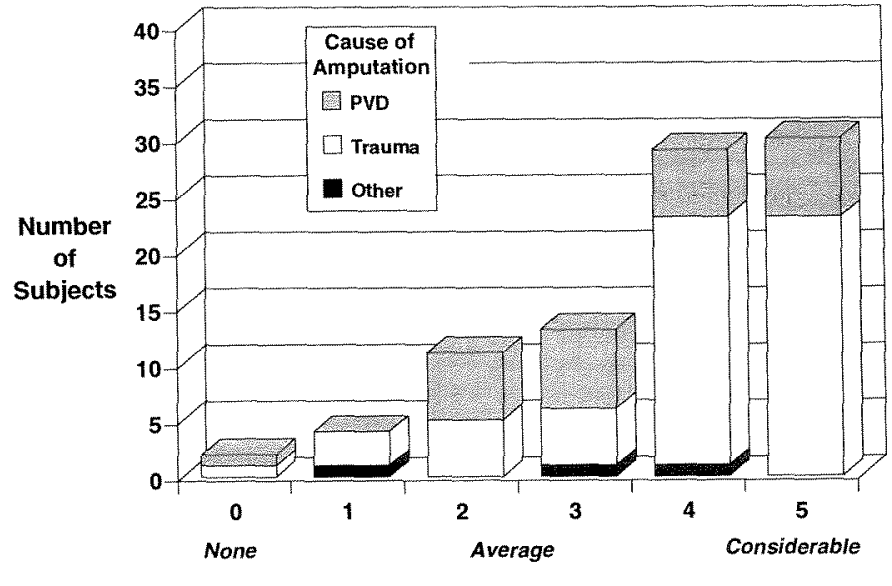

Distal Tissue Pressure Tolerance

Figure 17.

AFMA test subjects' residual limb distal tissue pressure tolerance categorized by cause of amputation.

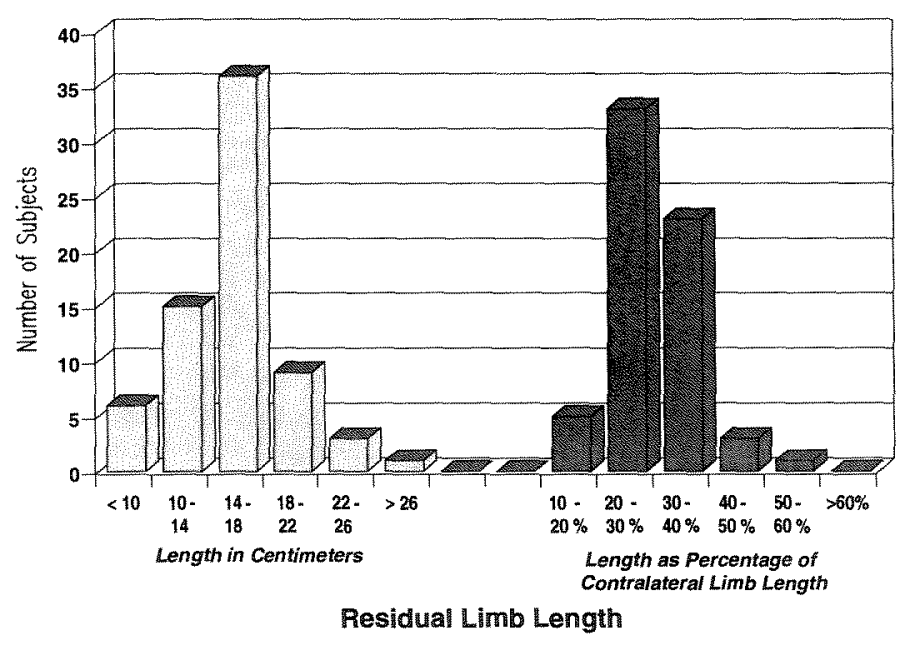

Figure 18.

AFMA test subjects' residual limb length in centimeters and as a relative percentage of their contralateral limb length.

stress tolerance levels. This proved to be contrary to the notion popular among some prosthetists that dysvascular amputees are less tolerant of residual limb loading than are traumatic amputees (assuming all stress magnitudes and gradients are below the levels causing stasis and chronic tissue trauma). Although average threshold levels of the stresses that cause stasis and that cause trauma are not generally known for amputees categorized by cause of amputation, from the available data it appears that threshold values vary considerably among 
groups $(17,18)$. PVD patients as a group may indeed have lower stasis and trauma thresholds, on the average, than other types of amputees, but this should not affect their loading tolerance as long as peak stresses and gradients remain below the respective threshold levels. This was felt to be the case for the subjects tested in the AFMA Program.

There was a spread in the distribution of the test subject residual limb lengths, ranging from $8 \mathrm{~cm}$ to $38 \mathrm{~cm}$ (Figure 18). The majority of the AFMA subjects, however, had residual limbs of $14.6 \mathrm{~cm}$, $\pm 4.3 \mathrm{~cm}$. This corresponded to residual limb lengths between 10 and 60 percent of the subjects" respective contralateral limb lengths, with the majority of the subjects having residual limb lengths between 25 and 34 percent of the length of their contralateral limbs. The majority of the subjects' residual limbs were either cylindrical (51 percent of the test subjects) or conical ( 42 percent of the test subjects) in shape, and had an average amount of soft tissue (67 percent of the subjects) of average durometer and elasticity ( 76 percent of the subjects), as shown in Tables 1a and 1b. These characteristics are representative of the category of residual limbs for which the patellar-tendon-bearing prosthesis was developed (9). It is of further interest to note that residual limb shape, amount, and relative durometer of residual limb soft tissue were relatively stable among the AFMA subjects who were long-term amputees (i.e., those subjects with 10 or more years since amputation). In the distribution of AFMA subjects with respect to residual limb shape, amount, and relative durometer of residual limb soft tissue, versus years since amputation, there is a trend in the first 1 to 5 years following amputation for the number of subjects with cylindrical and conical residual limbs to increase, for the number of subjects with an average amount of residual limb soft tissue to increase, and for the number of subjects with residual limb tissue of average firmness and elasticity to increase (Figures 19, 20, and 21). This corresponds to the general healing and stabilizing of residual limbs of persons with $\mathrm{BK}$ amputation, with atrophying of the pretibial and calf ankle/foot musculature. Beyond this, there were no trends apparent in the subject measurement data evidencing further change with increasing time since amputation in residual limb shape, amount of residual limb soft tissue, or decreasing residual limb tissue durometer and elasticity. Thus, the chronic

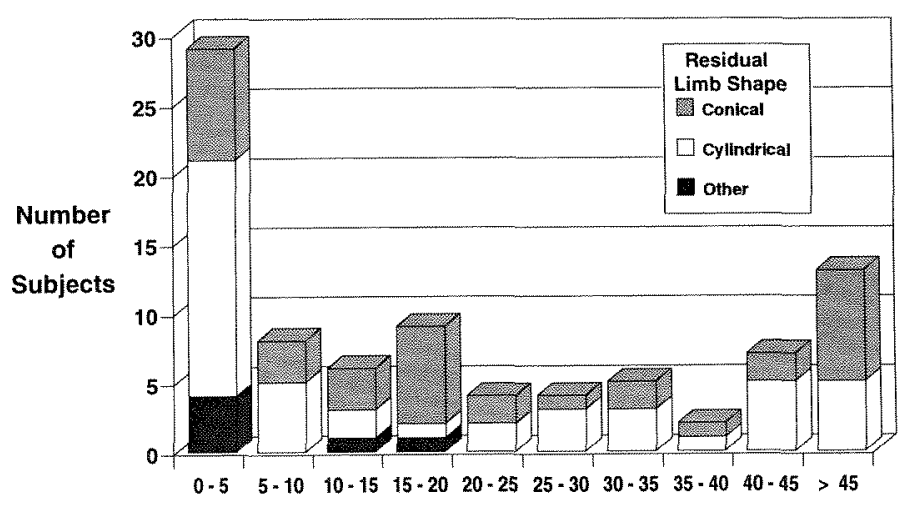

Years Since Amputation

Figure 19.

AFMA test subjects' residual limb shape categorized by the number of years since the subjects' amputation.

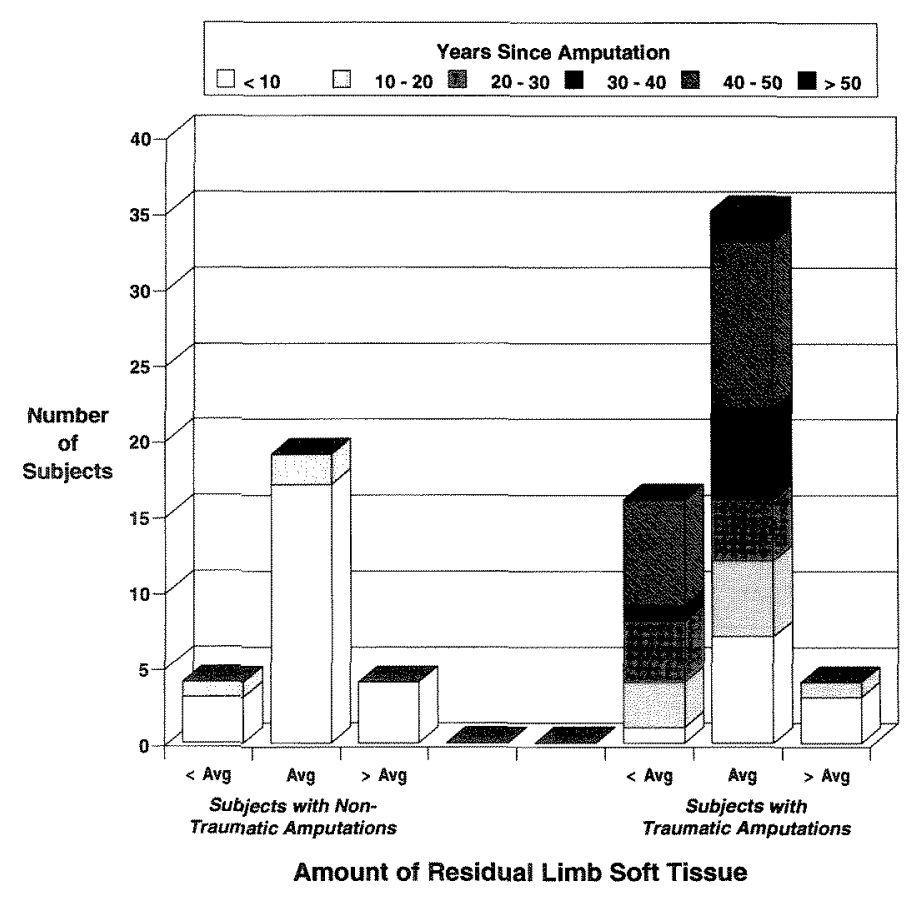

Figure 20.

AFMA test subjects' amount of residual limb soft tissue, categorized by cause of amputation and the number of years since amputation.

syndrome, believed to be prevalent before World War II, of BK amputation, prosthesis procurement and usage, chronic residual limb atrophy, residual $\operatorname{limb}$ breakdown, and eventual reamputation at the AK level, was not statistically evident among the AFMA test subjects. 


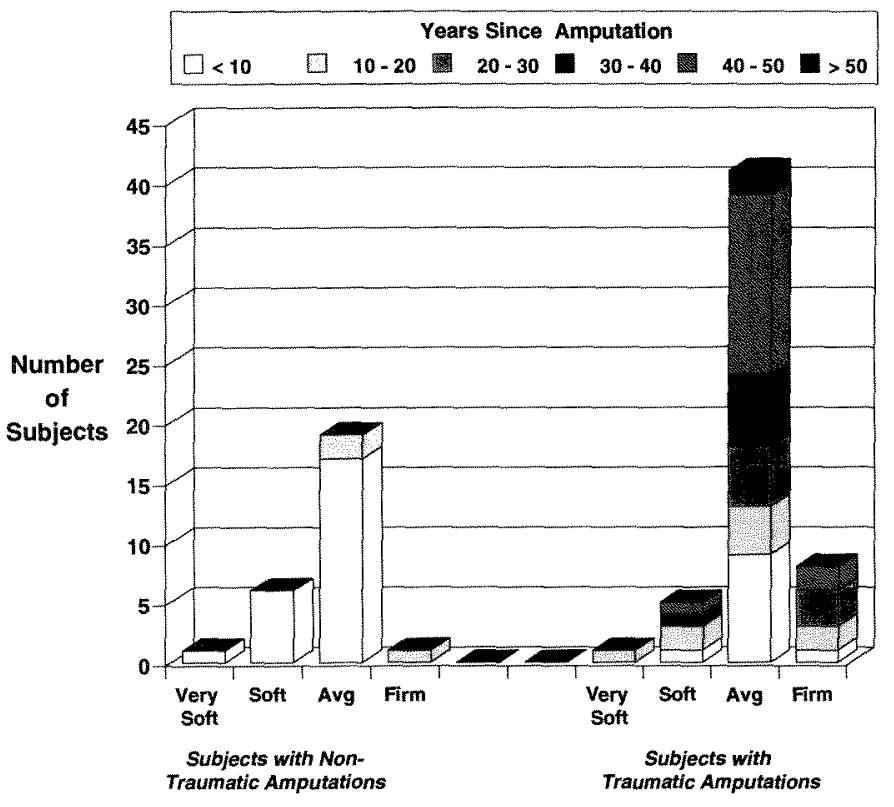

Residual Limb Soft Tissue Type

Figure 21.

AFMA test subjects' residual limb soft tissue type (relative durometer) categorized by number of years since amputation and cause of amputation.

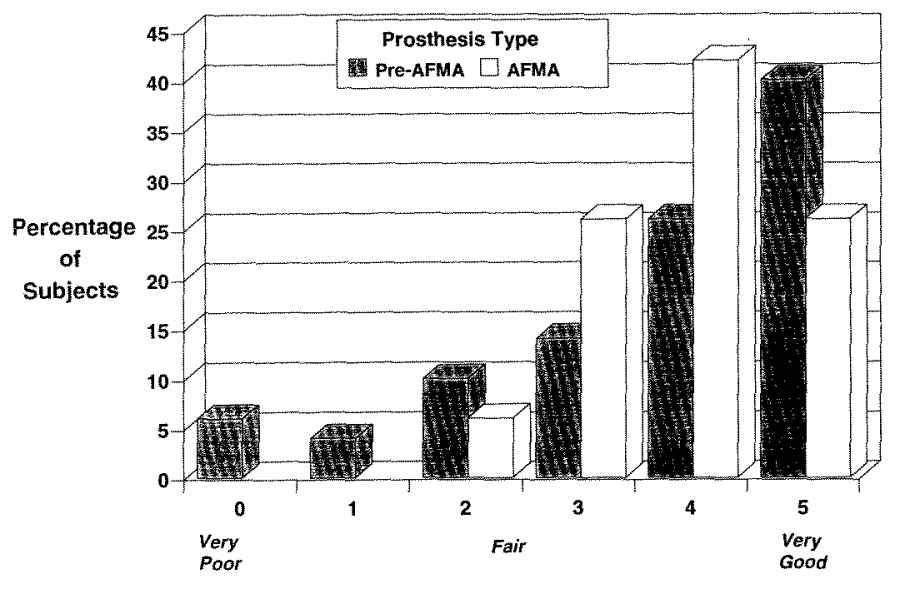

Prosthesis Overall Satisfaction Rating

Figure 22.

AFMA test subjects' ratings of the degree of their overall satisfaction with their conventional, pre-AFMA prostheses and with their AFMA CASD/CAM prostheses.

The majority of the test subjects' original, pre-AFMA prostheses were exoskeletal (68 percent), with soft socket liners (86 percent), and either PTB cuff (41 percent) or supracondylar suspension (31 percent), with SACH (38 percent) or energy storing
SACH (49 percent) feet. The prostheses had an average weight of $4.0 \mathrm{lb}$, with a standard deviation of $\pm 0.9 \mathrm{lb}$. The pre-AFMA prosthetic sockets had average anteroposterior (AP) dimensions at medial tibial plateau level of $9.2 \mathrm{~cm}$, with a standard deviation of $\pm 1.1 \mathrm{~cm}$, and average mediolateral (ML) dimensions at the apex of the femoral condyles of $10.6 \mathrm{~cm}$, with a standard deviation of $\pm 1.0 \mathrm{~cm}$. The subjects' pre-AFMA sockets were aligned with an average flexion of $5.6^{\circ}$, a median flexion of $5.0^{\circ}$, and a standard deviation of $\pm 4.3^{\circ}$. Similarly, the subjects' pre-AFMA sockets were aligned with an average of $3.6^{\circ}$ of adduction, a median of $3.0^{\circ}$ of adduction, and a standard deviation of $\pm 3.4^{\circ}$. The subjects' pre-AFMA prosthetic sockets were positioned relative to their prosthetic feet with an average anteroposterior position (socket sagittal midline relative to the ankle center line) of $1.3 \mathrm{~cm}$ anterior, a median of $2.0 \mathrm{~cm}$ anterior, and a standard deviation of $\pm 3.0 \mathrm{~cm}$; and with an average mediolateral position (socket coronal midline relative to the heel center line) of $0.0 \mathrm{~cm}$ inset, a median of $0.0 \mathrm{~cm}$, and a standard deviation of $\pm 1.5 \mathrm{~cm}$. In addition, the subjects reported their previous, pre-AFMA prostheses lasted, on the average, approximately 3.6 years, with a median of 3.0 years and a standard deviation of \pm 2.4 years, and that it required, on the average, about 2.5 months from time of prescription to time of delivery before they obtained a finished, conventionally designed and manufactured (non-AFMA) prosthesis. Further statistics regarding the test subjects' prosthetics characteristics are summarized in Table $\mathbf{2 a}$ and Table $\mathbf{2 b}$.

As seen in Figure 22, 40 percent of the AFMA test subjects rated the degree of their overall satisfaction with their pre-AFMA prostheses as being very good. Another 40 percent of the subjects rated their overall satisfaction with their pre-AFMA prostheses as good to fair. Fourteen percent of the subjects rated their overall satisfaction with their pre-AFMA prostheses as fair to poor, and 6 percent of the subjects rated their pre-AFMA prostheses overall as very poor. With respect to specific characteristics, 35 percent of the test subjects rated the fit of their pre-AFMA prosthetic sockets as being very good, 41 percent rated their pre-AFMA socket fit as good to fair, 17 percent as fair to poor, and 7 percent as very poor (Figure 23). At the same time, 33 percent of the subjects rated their pre- 
Table 2a.

AFMA test subject prosthetics characteristics.

\begin{tabular}{lcccc}
\hline Characteristic & $\begin{array}{c}\text { Number of Subjects } \\
\text { Pre-AFMA } \\
\text { Prosthesis }\end{array}$ & $\begin{array}{c}\text { PFMA } \\
\text { Prosthesis }\end{array}$ & $\begin{array}{c}\text { Pre-AFMA } \\
\text { Prosthesis }\end{array}$ & $\begin{array}{c}\text { AFMA } \\
\text { Prosthesis }\end{array}$ \\
\hline SOCKET TYPE & & & & \\
$\quad$ Hard & 13 & 4 & 14 & 6 \\
Soft & 77 & 66 & 86 & 94 \\
PROSTHETIC FOOT TYPE & & & & \\
Single Axis & 2 & 2 & 2 & 3 \\
Multi-Axis & 6 & 2 & 7 & 3 \\
SACH & 34 & 26 & 38 & 37 \\
Energy Storing SACH & 44 & 39 & 49 & 56 \\
Energy Storing Leaf Spring & 3 & 1 & 3 & 1 \\
PROSTHESIS TYPE & & & & \\
Exoskeletal & 62 & 15 & 69 & 21 \\
Endoskeletal & 28 & 55 & 31 & 79 \\
PROSTHESIS SUSPENSION & & & & \\
METHOD & & & & 6 \\
PTB Cuff & 27 & 43 & 30 & 61 \\
PTB Cuff \& Waist Belt & 10 & 5 & 11 & 7 \\
Waist Belt & 28 & 0 & 2 & 0 \\
Supracondylar & 10 & 3 & 31 & 4 \\
Suction & 11 & 4 & 2 & 6 \\
Sleeve & 13 & 11 & 12 \\
Other & & 2 & & \\
\hline \hline
\end{tabular}

AFMA prostheses as being very comfortable, 58 percent rated their pre-AFMA prostheses' comfort as being good to fair, 7 percent as fair to poor, and 2 percent as very uncomfortable. Hence, 76 percent of the test subjects were very to fairly satisfied with their pre-AFMA prosthetic socket fit, and 24 percent were fairly to very unsatisfied. Ninety-one percent of the subjects felt their pre-AFMA prostheses were very to fairly comfortable, and 9 percent felt they were fairly to very uncomfortable. The subjects' ratings with respect to the other prosthesis categories evaluated are shown in Figure 23. It is of interest to note that the test subjects' prosthesis evaluations did not evidence a one-to-one correspondence between socket fit and socket comfort, viz., 91 percent of the subjects felt their pre-AFMA prosthetic sockets were very to fairly comfortable, while only 76 percent of the subjects felt the fit of their pre-AFMA sockets was very good to fair (Figure 24). That the subjects' ratings of their prostheses' comfort and fit lay to the left (the comfort side) of the diagonal line from the point (comfort $=0$, fit $=0$ ) to the point $($ comfort $=5$, fit $=5$ ) in the horizontal "Comfort Rating versus Fit Rating" plane in Figure 24 (the comfort rating equals fit rating line), is an indication that comfort is a necessary but not a sufficient condition for good socket and prosthesis fit. Calculation of the sample correlation coefficient between comfort and fit further corroborates this. A value of 0.754 was obtained for the sample correlation coefficient between the test subjects' ratings of their pre-AFMA sockets' comfort and fit, indicating a strong correlation between, but not an equivalence of, socket/prosthesis comfort and fit ratings. This implies that a positive answer to the query, "How does your socket/prosthesis feel?"' indicates that although a patient finds a socket/prosthesis to be comfortable, it should not be interpreted as an indication that he/she feels the socket/prosthesis fit is also acceptable.

The subjects' ratings of their original, preAFMA prostheses, together with their medical, physiological, and prosthetics histories, and prescribed biomechanical and prosthetics measurements were obtained at the time the subjects were first 
Table $2 \mathrm{~b}$.

AFMA test subject prosthetics characteristics.

\begin{tabular}{|c|c|c|c|c|c|}
\hline Characteristic & Median & Avg. & SD & Min. & Max. \\
\hline \multicolumn{6}{|l|}{ Prosth. Weight (lbs) } \\
\hline Pre-AFMA & 4 & 4 & 0.9 & 2.0 & 7.0 \\
\hline AFMA & 3 & 3.5 & 0.9 & 2.5 & 7.5 \\
\hline \multicolumn{6}{|l|}{ Avg. Prosth. Lifetime (yr) } \\
\hline Pre-AFMA & 3 & 3.6 & 2.4 & 0.3 & 2 \\
\hline AFMA & --- & -- & -- & - & -- \\
\hline \multicolumn{6}{|c|}{ Avg. Time for Prosth. Provision (mo) } \\
\hline Pre-AFMA & 2 & 2.5 & 2.3 & 0.3 & 12 \\
\hline AFMA & -- & -- & -- & -- & - \\
\hline \multicolumn{6}{|l|}{ Amt. Socket Flexion (deg) } \\
\hline Pre-AFMA & 5 & 5.6 & 4.3 & -2 & 20 \\
\hline AFMA & 5 & 5.7 & 3.7 & 0 & 25 \\
\hline Diff. in Socket Flexion (deg) & & & & & \\
\hline$[($ Pre-AFMA $)$ - (AFMA) $]$ & 2 & 2.7 & 3.1 & 0 & 17 \\
\hline \multicolumn{6}{|l|}{ Amt. Socket Adduction (deg) } \\
\hline Pre-AFMA & 3 & 3.6 & 3.4 & -2 & 15 \\
\hline AFMA & 4 & 3.5 & 2.5 & 0 & 9 \\
\hline \multicolumn{6}{|l|}{ Diff. in Socket Adduction (deg) } \\
\hline$[(\operatorname{Pre}-\mathrm{AFMA})-(\mathrm{AFMA})]$ & 1 & 1.8 & 2.3 & 0 & 10 \\
\hline \multicolumn{6}{|l|}{ AP Foot Position $(\mathrm{cm})$} \\
\hline Pre-AFMA & 2 & 1.3 & 3.0 & -8.3 & 8.2 \\
\hline AFMA & 2 & 0.6 & 3.4 & -9.5 & 7.6 \\
\hline \multicolumn{6}{|l|}{ Diff. in AP Foot Position $(\mathrm{cm})$} \\
\hline [(Pre-AFMA) - (AFMA)] & 1.3 & 2 & 2.2 & 0 & 9.5 \\
\hline \multicolumn{6}{|l|}{ ML Foot Position $(\mathrm{cm})$} \\
\hline Pre-AFMA & 0 & 0 & 1.5 & -5.1 & 2.5 \\
\hline AFMA & 0 & -0.2 & 1.5 & -4 & 2.5 \\
\hline \multicolumn{6}{|l|}{ Diff. in ML Foot Position (cm) } \\
\hline$[($ Pre-AFMA) - (AFMA) $]$ & 0.6 & 1.2 & 1.3 & 0 & 4.5 \\
\hline \multicolumn{6}{|c|}{ AP Socket Dimension at MTP $(\mathrm{cm})$} \\
\hline Pre-AFMA & 9.0 & 9.2 & 1.1 & 7.3 & 13.7 \\
\hline AFMA & 9.1 & 9.2 & 1.1 & 6.5 & 12.0 \\
\hline \multicolumn{6}{|c|}{ Diff. in AP Socket Dimension $(\mathrm{cm})$} \\
\hline$[($ Pre-AFMA $)-($ AFMA $)]$ & 0.4 & 0.6 & 0.6 & 0 & 3.2 \\
\hline \multicolumn{6}{|c|}{$\begin{array}{l}\text { ML Socket Dimension at Femoral } \\
\text { Condyles }(\mathrm{cm})\end{array}$} \\
\hline Pre-AFMA & 10.7 & 10.6 & 1.1 & 8.1 & 13.7 \\
\hline AFMA & 10.5 & 10.4 & 0.9 & 8.0 & 12.3 \\
\hline \multicolumn{6}{|l|}{ Diff. ML Socket Dimension (cm) } \\
\hline$[($ Pre-AFMA $)-($ AFMA $)]$ & 0.3 & 0.5 & 0.5 & 0 & 2.4 \\
\hline
\end{tabular}




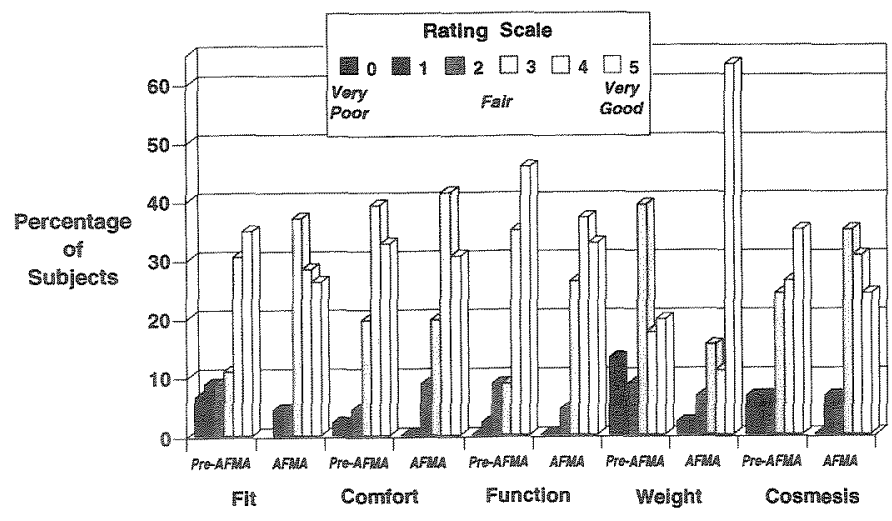

Prosthesls Characleristics Evalualed

Figure 23.

AFMA test subjects' ratings of their conventional, pre-AFMA prostheses and their AFMA CASD/CAM prostheses.

enrolled in the AFMA Program. The subjects', and the respective AFMA prosthetists', evaluations of the AFMA CASD/CAM prostheses were obtained only after the subjects had successfully worn their CASD/CAM prostheses without complications for 1 month. In this way, any potential bias in favor of either the pre-AFMA or AFMA prostheses and sockets was minimized.

The definitive AFMA prostheses provided to the test subjects when successful socket designs and fits were achieved were mostly endoskeletal (79 percent), with soft socket liners (94 percent), and with PTB cuff suspension (61 percent), and SACH (37 percent) or energy storing SACH (56 percent) feet. Endoskeletal AFMA prostheses were used primarily for economies in labor and fabrication time. Soft socket liners and PTB cuff suspension were used in the majority of cases because this was the type of socket and type of suspension for which the UCL-BC CASD socket template tested was designed. When subjects needed or expressed a very strong preference for a different type of socket, method of suspension, or prosthesis, they were accommodated, if possible (Tables $2 a$ and $2 b$ ). Although this usually required additional work, adapting the socket design slightly in the case of hard sockets and alternate suspension methods, the

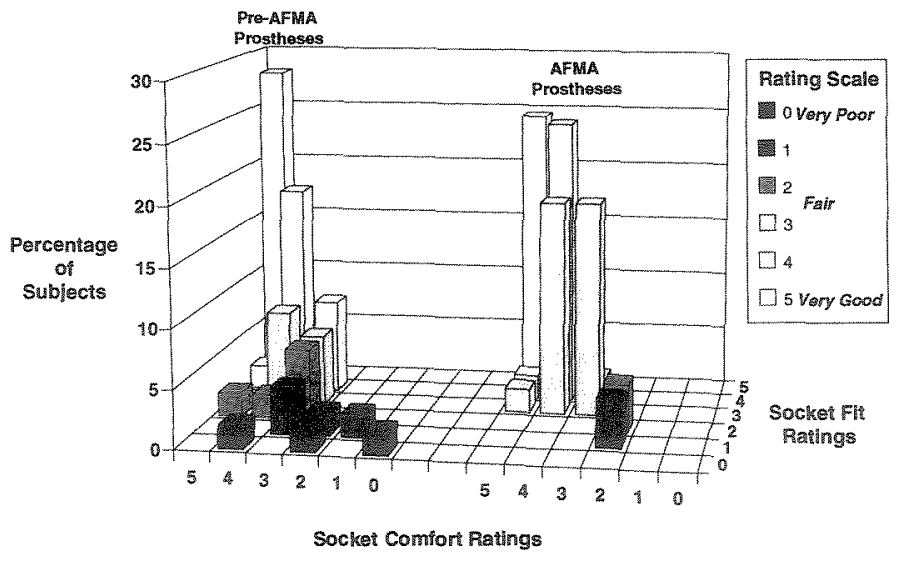

Tigure 24.

AFMA test subjects' ratings of the degree of comfort and quality of fit of their conventional, pre-AFMA prostheses and their AFMA CASD/CAM prostheses.

ability to do so evidenced the flexibility and the capabilities of the CASD systems. To minimize any functional differences between pre-AFMA and AFMA prostheses, subjects were given the same type of prosthetic foot contained in their pre-AFMA prostheses, unless they expressed a very strong preference for a different type. The AFMA prostheses had an average weight of $3.4 \mathrm{lb}$, with a median weight of $3.0 \mathrm{lb}$ and a standard deviation of \pm 0.9 1b. Because the majority of the AFMA prostheses were endoskeletal, 79 percent compared with 32 percent of the subjects" pre-AFMA prostheses, the AFMA prostheses were generally lighter. Every subject for whom this was the case rated this as an improvement, although two subjects felt their AFMA prostheses at $2.7 \mathrm{lb}$ were still too heavy. Prosthesis weight affects socket/residual limb loading predominantly during the swing phase of gait. Functionally, assuming proper prosthesis suspension, the major differences arising from variations in prosthesis weight are principally in the energy and strength required to accelerate and decelerate the prosthesis at the beginning and end of the swing phase of the gait cycle. The associated socket/ residual limb loads occurring during the swing phase of gait with different weight prostheses may vary in magnitude but basically have the same distributions, 
Journal of Rehabilitation Research and Development Vol. 29 No. 4 Fall 1992

and have magnitudes below the socket/residual limb loading that occurs during stance. In the AFMA clinical trials, the lower weight of the AFMA prostheses did not observably affect socket/residual limb loading during stance or swing. It was felt, therefore, that the effects of varying prosthesis construction (endoskeletal versus exoskeletal) on the testing and evaluation of the fit, comfort, and function of the AFMA CASD sockets were minimal.

The amount of time the test subjects had worn their present pre-AFMA prostheses ranged from 0.25 years to 12 years, so the fit, alignment, function, etc., of the pre-AFMA prostheses were not optimal in all cases. In addition, the prosthetists who designed, fit, and aligned many of the subjects' pre-AFMA prostheses were not the same as the prosthetists who designed, fit, and aligned their AFMA prostheses, so the criteria used to establish the original, pre-AFMA prostheses' fit and alignment were not always known. Therefore, it was decided that the AFMA sockets and prostheses should be designed, fit, and aligned using the established criteria taught in the major university prosthetics education programs $(10,11,12)$, without reference to the subjects' pre-AFMA prosthetics parameters. It was felt that this approach would yield more consistent results among the AFMA Centers and the AFMA prosthetists. It was also felt that the results so obtained would be more representative of the results arising from clinical application of the CASD/CAM systems in the prosthetics field in the United States, in general.

\section{UCL-BC CASD/CAM System clinical testing and evaluation results}

The UCL-BC CASD sockets had average anteroposterior (AP) dimensions at medial tibial plateau level of $9.2 \mathrm{~cm}$, with a standard deviation of $\pm 1.1 \mathrm{~cm}$, and average mediolateral (ML) dimensions at the apex of the femoral condyles of 10.4 $\mathrm{cm}$, with a standard deviation of $\pm 0.9 \mathrm{~cm}$. The subjects' UCL-BC CASD sockets were aligned with an average of $5.7^{\circ}$ of flexion, a median of $5.0^{\circ}$ of flexion, and a standard deviation of $\pm 3.7^{\circ}$. Similarly, the subjects' UCL-BC CASD sockets were aligned with an average of $3.5^{\circ}$ of adduction, a median of $4.0^{\circ}$ of adduction, and a standard deviation of $\pm 2.5^{\circ}$. The subjects' UCL-BC CASD sockets were positioned relative to their prosthetic feet with an average anteroposterior position (socket sagittal midline relative to the ankle center line) of $0.6 \mathrm{~cm}$ anterior, a median of $2.0 \mathrm{~cm}$ anterior, and a standard deviation of $\pm 3.4 \mathrm{~cm}$; and with an average mediolateral position (socket coronal midline relative to the heel center line) of $0.2 \mathrm{~cm}$ inset, a median of $0.0 \mathrm{~cm}$, and a standard deviation of $\pm 1.5 \mathrm{~cm}$.

The average differences between the pre-AFMA and AFMA prosthetics parameters that arose are shown in Tables $\mathbf{2 a}$ and $\mathbf{2 b}$. On the average, the median and mean differences between the preAFMA and AFMA parameters are relatively small. Those instances of substantial differences occurred, in general, when the subjects' pre-AFMA parameters differed substantially from the respective preAFMA mean values. In almost all cases, the AFMA parameters were found to lie closer to the respective AFMA mean and median values, thus resulting in tighter parametric distributions of the AFMA parameters than of the corresponding pre-AFMA parameters. In addition, 48 percent of the test subjects were reported by the AFMA prosthetists to exhibit one or more gait deviations with their pre-AFMA prostheses, whereas only 10 percent of the subjects were reported to have gait deviations with their AFMA prostheses.

As seen in Figure 22, 25 percent of the subjects rated their overall satisfaction with their AFMA UCL-BC CASD/CAM prostheses as being very good. Sixty-nine of the subjects rated their overall satisfaction with their UCL-BC CASD/CAM prostheses as good to fair, and 6 percent of the subjects gave their UCL-BC CASD/CAM prostheses fair to poor overall ratings. None of the test subjects gave their UCL-BC CASD/CAM prostheses an overall satisfaction rating of very poor. With respect to specific characteristics, 26 percent of the subjects rated the fit of their UCL-BC CASD/CAM prostheses as being very good, 65 percent rated the fit as being good to fair, 9 percent as being fair to poor, and none of the subjects felt the UCL-BC CASD/CAM prostheses fit very poorly. Thirty percent of the subjects rated their UCL-BC CASD/CAM prostheses as being very comfortable, 61 percent rated the comfort as being good to fair, and 9 percent rated the comfort as fair. None of the subjects felt their UCL-BC CASD/CAM prostheses were uncomfortable to very uncomfortable. Hence, 94 percent of the test subjects were very to fairly satisfied overall with their UCL-BC CASD/CAM prostheses, and only 6 percent were fairly satisfied 


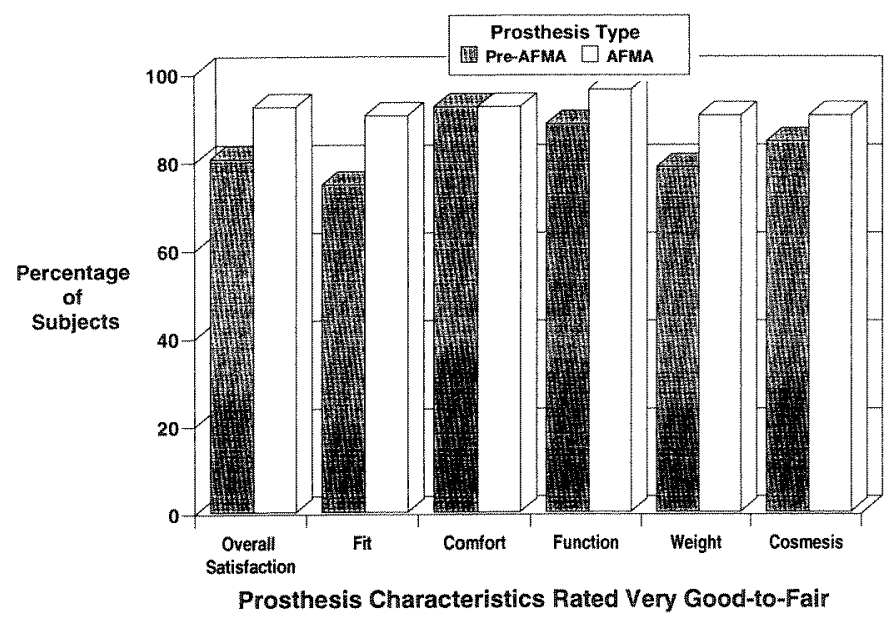

Figure 25.

Percentage of AFMA test subjects who rated the specified characteristics of their pre-AFMA prostheses and their AFMA prostheses very good to fair $(5,4$, or 3$)$.

to unsatisfied, compared with 80 percent who were very to fairly satisfied with their pre-AFMA prostheses and 20 percent who were not.

As seen in Figure 25 and Figure 26, more of the test subjects gave their UCL-BC CASD/CAM prostheses very good to fair ratings with respect to overall satisfaction, fit, and function, than gave their conventional, pre-AFMA prostheses very good to fair ratings. This is also true with respect to the subjects' ratings of weight and cosmesis of their prostheses. An equal number of subjects rated their pre-AFMA and UCL-BC CASD/CAM prostheses very good to fair with respect to comfort. Restricting the results to those subjects who had been fit and had worn their present pre-AFMA prostheses for 1 year or less, there were more subjects who rated their relatively new pre-AFMA prostheses as fair to very poor than rated their UCL-BC AFMA CASD/CAM prostheses as fair to poor. None of the subjects rated their UCL-BC CASD/CAM prostheses as very poor. Hence, the best pre-AFMA prostheses designed, fabricated, and fit by skilled prosthetists using conventional prosthetics techniques achieved the highest overall satisfaction rating and the best fit, comfort, and function ratings by more subjects than did the AFMA prostheses designed, manufactured, and fit using the UCL-BC CASD/CAM system. More subjects, however, were satisfied with, and gave very good to fair ratings (5, 4 , or 3) to, their UCL-BC CASD/CAM prostheses,

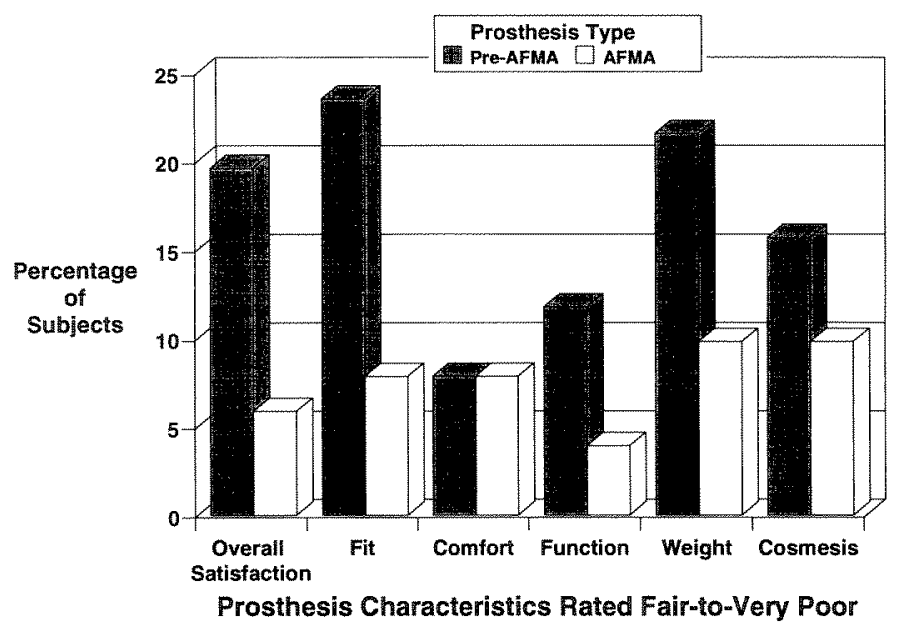

Figure 26.

Percentage of AFMA test subjects who rated the specified characteristics of their pre-AFMA prostheses and their AFMA prostheses fair to very poor $(2,1$, or 0$)$.

especially the fit of their UCL-BC CASD/CAM sockets, than gave very good to fair ratings to their conventionally designed and produced, pre-AFMA prostheses. Furthermore, the "worst fits" obtained with the UCL-BC CASD/CAM system were not rated as bad as the worst fits produced by some prosthetists using conventional techniques.

Conditioning the subjects' ratings of their prostheses' characteristics on the degree of their overall satisfaction with their prostheses, it is seen that even though some subjects gave their preAFMA and/or AFMA prostheses very good to fair overall ratings, they were not completely satisfied with all aspects of their prostheses. Figure 27 shows the distributions of the test subjects' ratings of their pre-AFMA and UCL-BC AFMA prostheses' for those subjects with overall satisfaction ratings of very good to fair. Similarly, Figure $\mathbf{2 8}$ shows the distributions of prosthesis characteristic ratings for those subjects who gave their prostheses overall satisfaction ratings of fair to very poor. As seen in Figure 27, in those cases in which the subjects gave very good to fair overall ratings to their prostheses, but fair to very poor ratings to one or more of their prostheses' characteristics, the characteristics the subjects were most frequently dissatisfied with were weight, cosmesis, and fit, respectively. This was true for both pre-AFMA and AFMA prostheses. As seen in Figure 28, however, those subjects who gave their pre-AFMA prostheses fair to very poor overall 
ratings were most frequently dissatisfied with the fit of their pre-AFMA prostheses. After fit, the characteristics the subjects who gave their pre-AFMA prostheses fair to very poor overall ratings were most dissatisfied with, were the function of their pre-AFMA prostheses, then the weight, the comfort, and finally the cosmesis. In contrast, of the subjects who rated their UCL-BC CASD/CAM prostheses fair to poor overall, approximately an equal number of subjects was dissatisfied with each of the characteristics of the UCL-BC CASD/CAM prostheses. Fit and comfort were not the dominant concern of these subjects as they were with the subjects who were dissatisfied overall with their pre-AFMA prostheses. This corroborates the assertion that the least satisfactory fits obtained with the UCL-BC $\mathrm{CASD} / \mathrm{CAM}$ system were not as bad, in general, as those produced by some of the pre-AFMA prosthetists using conventional procedures.

To delineate those areas where the UCL-BC CASD/CAM system performed well and those areas where additional refinement and development were needed, the computer-aided socket design and manufacturing process was broken down into constituent parts and analyzed. As seen in Figure 29, an average of 3.8 check sockets per subject were required to obtain successful $C A S D / C A M$ socket designs and fits for the subjects tested in the AFMA clinical trials. The PRS prosthetists averaged 3.0 check sockets for the subjects they tested, the NU-VALMC prosthetists averaged 2.5 check sockets, and the NY DVAMC prosthetists averaged 4.5 check sockets. Figure 30 shows the number of subjects for which changes in the UCL-BC socket template were required to achieve successful socket designs and fits. Changes in an average of five of the UCL-BC socket template modification regions were required before successful socket designs and fits were obtained for the AFMA test subjects. The subjects tested by the PRS prosthetists required an average of four modification template changes, the NU-VALMC test subjects required an average of three template changes, and the NY DVAMC test subjects required an average of five template changes before acceptable socket designs and fits were achieved. Figure 31 shows the average change in the foci at each of the template modification regions that were required to achieve successful CASD/CAM socket designs and fits for the AFMA test subjects. Additional statistics regarding the

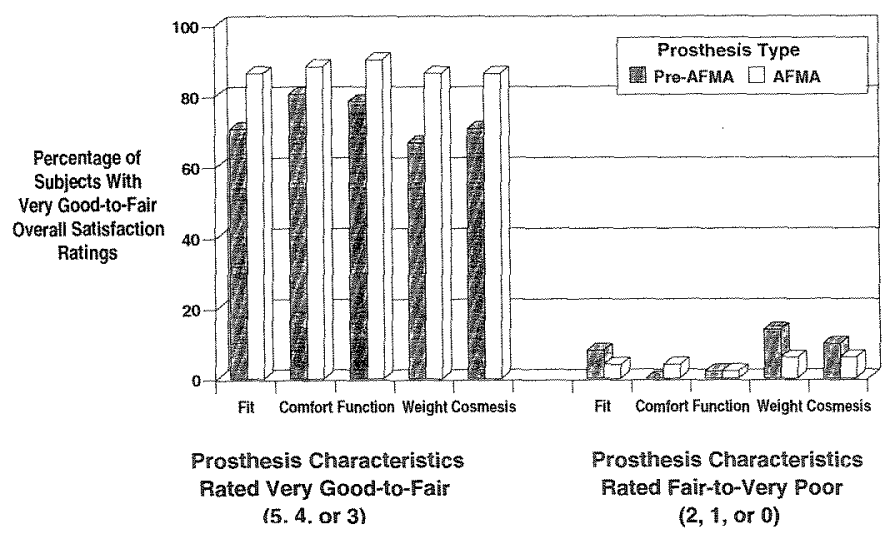

Figure 27.

Prosthesis characteristic ratings by the AFMA test subjects who gave their pre-AFMA or AFMA prostheses overall satisfaction ratings of very good to fair $(5,4$, or 3$)$.

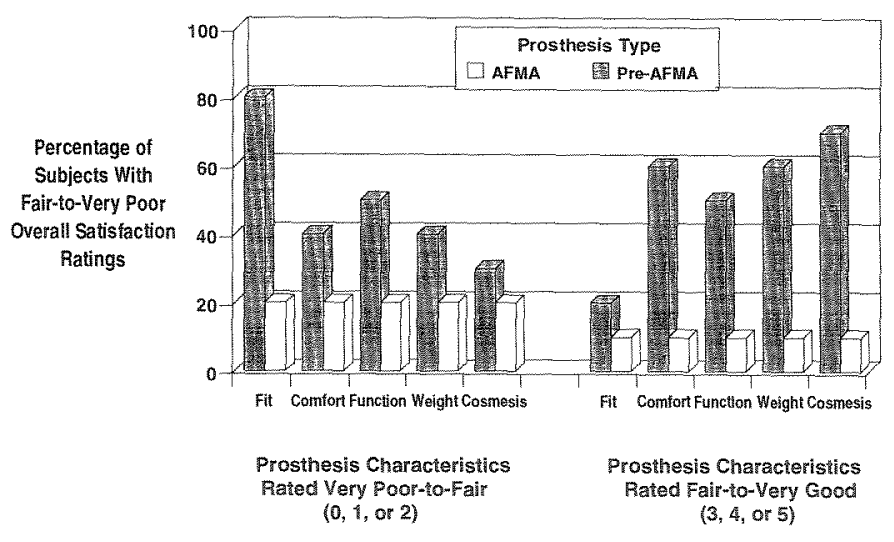

Figure 28.

Prosthesis characteristic ratings by the AFMA test subjects who gave their pre-AFMA or AFMA prostheses overall satisfaction ratings of fair to very poor $(2,1$, or 0$)$.

required UCL-BC template changes are given in Tables $3 a, 3 b$, and $3 \mathrm{c}$. Figure 32 shows the number of individual socket node modifications (changes in radii at the discrete points constituting the digitized residual limb measurement and socket models) that had to be made, in addition to changes in the template modification region foci, to obtain successful socket designs and fits. For the three AFMA Centers, an average of 254 individual socket nodes per subject had to be modified to achieve successful socket designs and fits. The PRS prosthetists modified an average of 16 individual socket nodes per subject, the NU-VALMC prosthetists modified an average of 22 individual socket nodes for the 


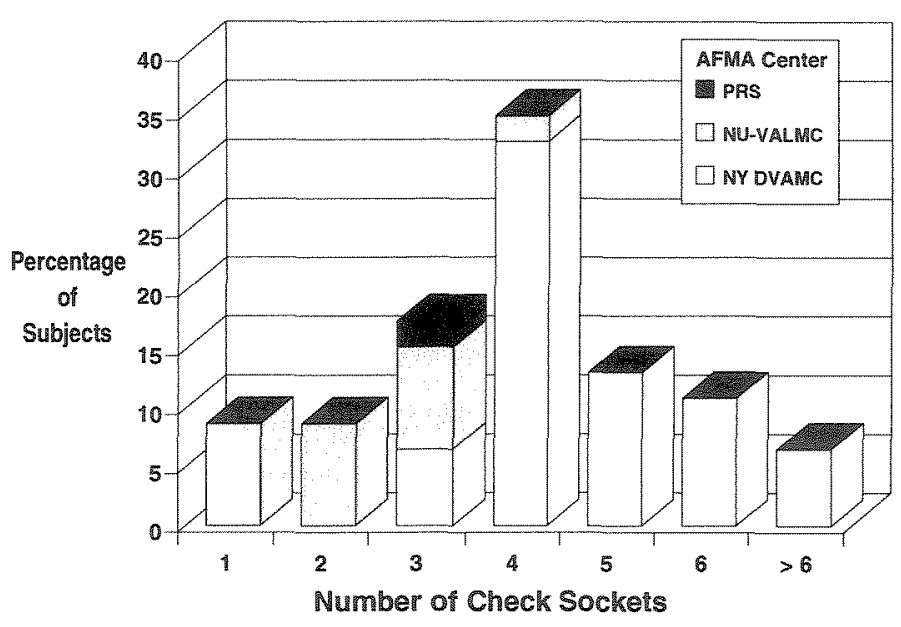

Figure 29.

Number of check sockets required to achieve successful socket designs and fittings with the UCL CASD/CAM System, categorized by AFMA Center.

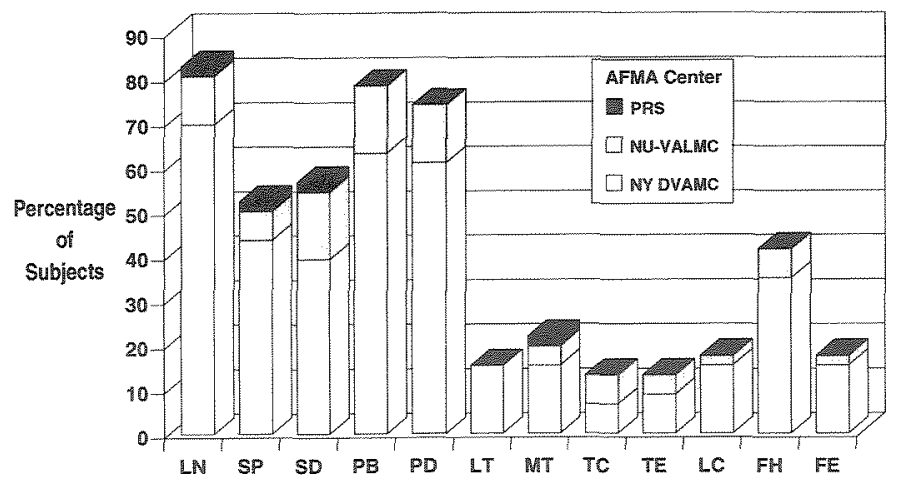

UCL-BC BK CASD TEMPLATE MODIFICATION REGIONS

Figure 30.

Percentage of AFMA test subjects for which changes in the foci of the UCL-BC CASD template modification regions were required to achieve successful socket designs and fittings, categorized by AFMA Center. The modification template regions are designated by: $L N$-residual limb length; SP-radial size change over proximal $1 / 3$ residual limb; SD-radial size change over distal $2 / 3$ of residual limb; PB - patellar bar; $P D-$ popliteal depression; LT-lateral tibial shaft flare; MT-medial tibial shaft flare; TC-tibial crest; TE-end of tibia; LClateral tibial condyle; $\mathrm{FH}$ - fibular head; $\mathrm{FE}$-end of fibia.

subjects they tested, and the NY DVAMC prosthetists modified an average of 365 individual socket nodes per subject before successful socket designs and fits were achieved.

To obtain a quantitative measure of the amount of work performed in designing, producing, and

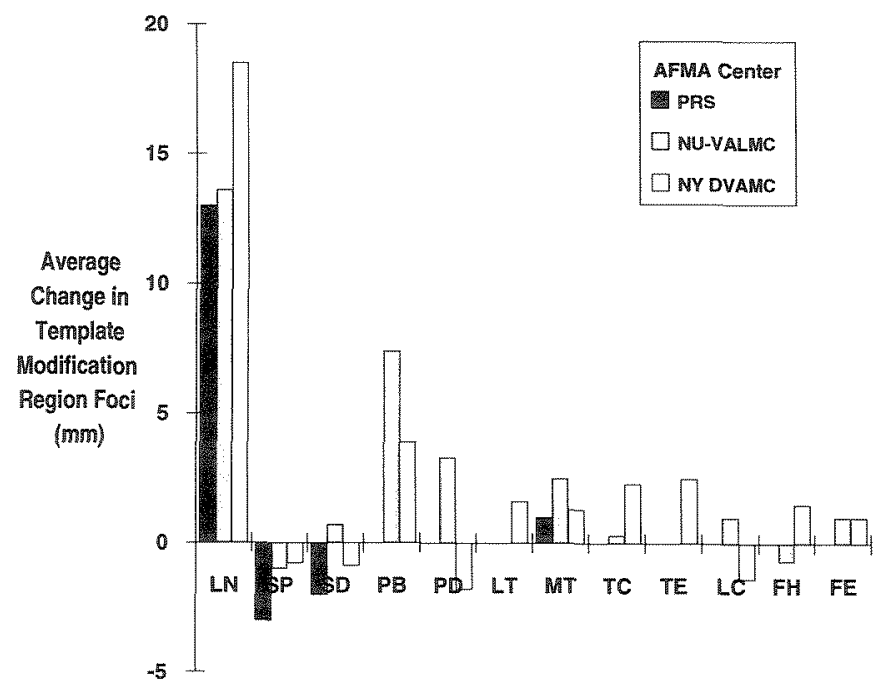

UCL-BC BK CASD Template Modification Regions

Figure 31.

Average change in the UCL-BC CASD template modification region foci required to achieve successful socket designs and fittings for the test subjects, categorized by AFMA Center.

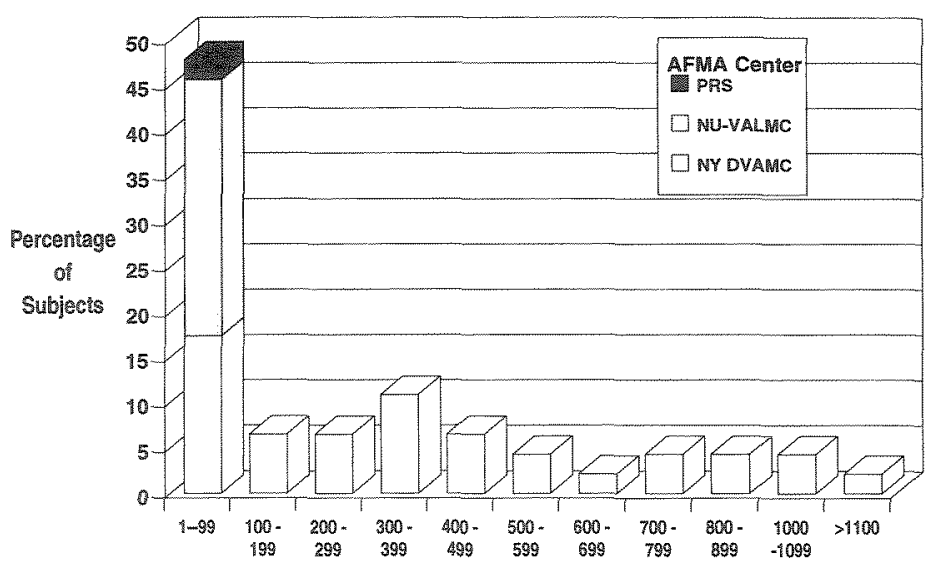

Number of Individual Socket Nodes Requiring Modification

Figure 32.

Number of individual socket nodes requiring radius changes, in addition to template region foci changes, to achieve successful UCL-BC CASD socket designs and fittings, categorized by AFMA Center.

fitting CASD/CAM sockets and prostheses, the AFMA investigators derived the time-productivity relationship given in Table 4 . Based on results obtained from clinical and laboratory tests, each task in the CASD/CAM socket/prosthesis design, production, and fitting process was assigned a 
Journal of Rehabilitation Research and Development Vol. 29 No. 4 Fall 1992

Table 3a.

Number of UCL-BC CASD design iterations (check sockets) required to achieve successful socket designs and fittings.

\begin{tabular}{cc}
\hline $\begin{array}{c}\text { Number of } \\
\text { Check Sockets }\end{array}$ & $\begin{array}{c}\text { Percentage of } \\
\text { Subjects Requiring } \\
\text { Specified Number of } \\
\text { Check Sockets }\end{array}$ \\
\hline One & $9 \%$ \\
Two & $9 \%$ \\
Three & $17 \%$ \\
Four & $35 \%$ \\
Five & $13 \%$ \\
Six & $11 \%$ \\
$>$ Six & $6 \%$ \\
\hline $\begin{array}{l}\text { Average Number of Check } \\
\text { Sockets Required }\end{array}$ & 3.8 \\
Standard Deviation in Number \\
of Check Sockets Required
\end{tabular}

weight representative of the average amount of time required to complete that task. Using this relation and $a$ posteriori information obtained from the UCL-BC CASD computer patient files on the type and number of times specific tasks were performed before successful CASD/CAM socket designs and fits were achieved, an estimate of the time required to design and produce successful CASD/CAM prostheses was obtained (Figure 33). These results provide an estimate of the productivity achieved with the UCL-BC CASD/CAM system during the AFMA clinical trials. The average work-time for the three AFMA centers for successful socket/prosthesis design, production, and fitting was 7.5 hours, with a standard deviation of \pm 1.8 hours, and a median of 7.6 hours (Figure 34). The activity attributed to be the principal contributor to longer work-times was individual socket node modification. The geometric primitive in the UCL-BC Computer Shape CASD software for individual socket node modification is simple and relatively straightforward. When multiple regions of 20 or more nodes required modification, however, the work-times grew asymptotically. As shown in Figure 35, the number of socket node modifications required by the AFMA test subjects varied considerably. Consequently, the distribution

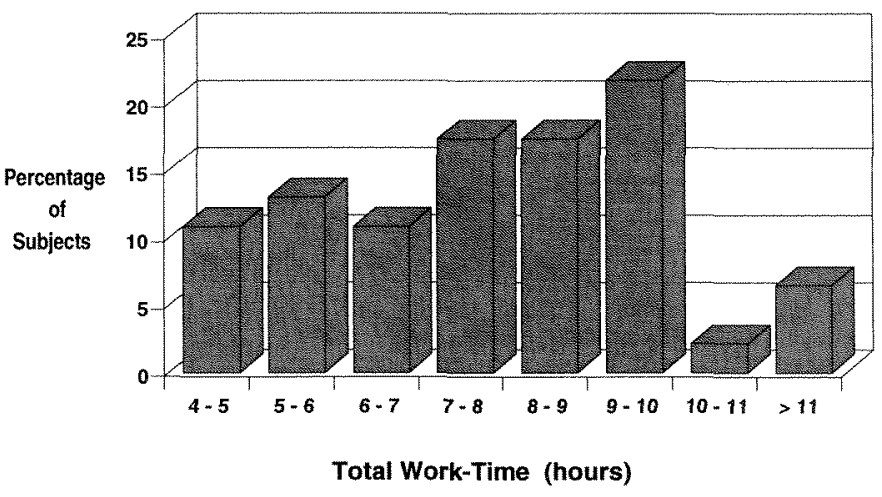

Figure 33.

Total work-time (personnel-hours) required for successful CASD/CAM socket and prosthesis design, production, and fitting.

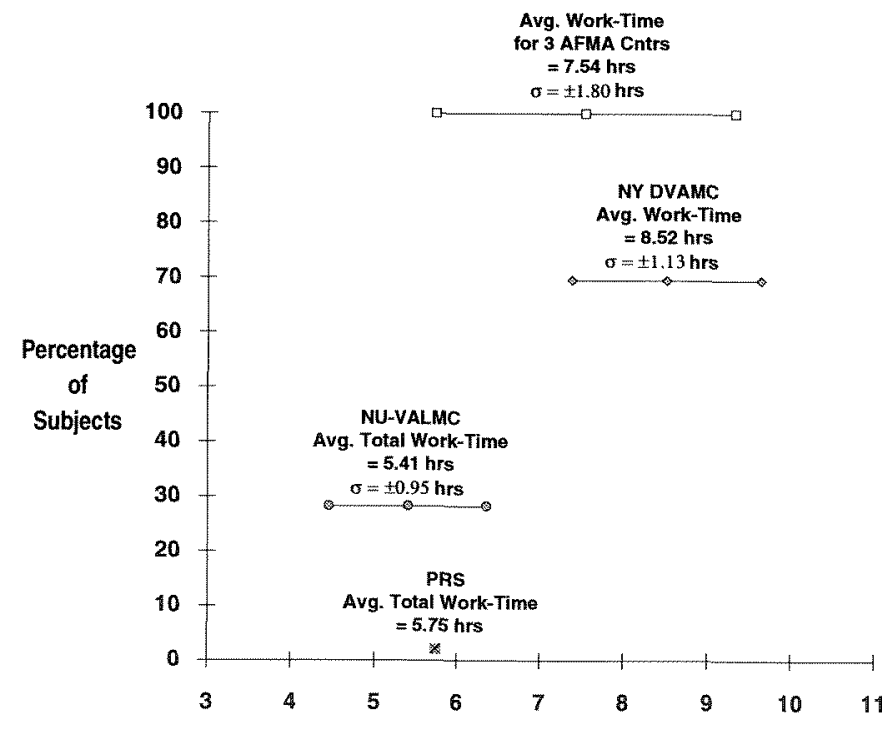

Average Total Work-Time (hours)

Figure 34 .

Average work-time (personnel-hours) required for successful CASD/CAM socket and prosthesis design, production, and fitting.

of work-times for CASD/CAM socket/prosthesis design, production, and fitting was dispersed, and extended well above the 5- to 6-hour range that might otherwise have constituted the average. Had the UCL-BC BK socket design templates provided a more accurate and intimate initial fit, the number of template regions and individual socket nodes that had to be modified would have been considerably reduced. This would have markedly decreased the 
Table 3b.

UCL-BC CASD modifications required for successful socket design and fitting.

\begin{tabular}{|c|c|c|c|}
\hline $\begin{array}{l}\text { CASD Template } \\
\text { Region }\end{array}$ & $\begin{array}{c}\text { Percentage of } \\
\text { Subjects Requiring } \\
\text { Change }\end{array}$ & $\begin{array}{c}\text { Average Change } \\
\text { From Standard } \\
\text { Template Value } \\
\text { (mm) }\end{array}$ & $\begin{array}{c}\text { Standard Deviation } \\
\text { of Changes in } \\
\text { Template Values } \\
\text { (mm) }\end{array}$ \\
\hline $\begin{array}{l}\text { Socket Length } \\
\text { (LN) }\end{array}$ & $83 \%$ & 14.61 & 9.70 \\
\hline $\begin{array}{l}\text { Socket Proximal Size } \\
\text { (SP) }\end{array}$ & $52 \%$ & -0.46 & 1.54 \\
\hline $\begin{array}{l}\text { Socket Distal Size } \\
\text { (SD) }\end{array}$ & $56 \%$ & -0.28 & 1.50 \\
\hline $\begin{array}{l}\text { Patellar Bar } \\
\text { (PB) }\end{array}$ & $78 \%$ & 3.57 & 3.02 \\
\hline $\begin{array}{l}\text { Popliteal Depression } \\
\text { (PD) }\end{array}$ & $74 \%$ & -0.65 & 3.16 \\
\hline $\begin{array}{l}\text { Lateral Tibial Shaft } \\
\text { Flare } \\
\text { (LT) }\end{array}$ & $15 \%$ & 0.24 & 0.97 \\
\hline $\begin{array}{l}\text { Medial Tibial Shaft } \\
\text { Flare } \\
\text { (MT) }\end{array}$ & $21 \%$ & 0.33 & 1.49 \\
\hline $\begin{array}{l}\text { Tibial Crest } \\
\text { (TC) }\end{array}$ & $14 \%$ & 0.17 & 0.85 \\
\hline $\begin{array}{l}\text { End of Tibia } \\
\text { (TE) }\end{array}$ & $13 \%$ & 0.22 & 0.99 \\
\hline $\begin{array}{l}\text { Lateral Tibial } \\
\text { Condyle } \\
\text { (LC) }\end{array}$ & $17 \%$ & 0.20 & 1.15 \\
\hline $\begin{array}{l}\text { Fibular Head } \\
\text { (FH) }\end{array}$ & $42 \%$ & 0.43 & 1.52 \\
\hline $\begin{array}{l}\text { End of Fibia } \\
\text { (FE) }\end{array}$ & $17 \%$ & 0.17 & 0.87 \\
\hline $\begin{array}{l}\text { Average Number of } \\
\text { Template Values } \\
\text { Changed Per Subject }\end{array}$ & 4.8 & $\begin{array}{l}\text { Standard Deviation } \\
\text { in No. of Template } \\
\text { Values Changed Per } \\
\text { Subject }\end{array}$ & 2.41 \\
\hline
\end{tabular}

number of check sockets and the AFMA prosthetists' work-time required, and would have considerably increased the CASD/CAM system productivity achieved.

To ascertain where refinements should be made in the UCL-BC CASD system to improve the quality of the CASD socket designs, to increase the system's productivity, and to gain insight for development of new CASD templates and design techniques, the test subjects' CASD files were analyzed.
For the subjects who completed the AFMA clinical trials, successfully wearing their AFMA prostheses for a period of at least 1 month, the changes in the UCL-BC modification template regions shown in Figure 31 and Tables $3 \mathbf{a}, \mathbf{3 b}$, and $\mathbf{3 c}$ were required. In addition, individual socket node modifications were found to be required: 1) to reduce the depth and width, and to smooth the contours of the patellar bar for 67 percent of the subjects; 2) to smooth the distal end of the socket for 61 percent of 
Table 3c.

UCL-BC CASD modifications required for successful socket design and fitting.

\begin{tabular}{lc}
\hline \multicolumn{1}{c}{$\begin{array}{c}\text { Regions Requiring } \\
\text { Node Modification }\end{array}$} & $\begin{array}{c}\text { Percentage of } \\
\text { Subjects Requing } \\
\text { Specified Socket } \\
\text { Node Modilications }\end{array}$ \\
\hline Patellar Bar & $67 \%$ \\
Distal End of Socket & $61 \%$ \\
Lateral Tibial Shaft & $59 \%$ \\
Medial Tibial Shaft & $52 \%$ \\
Popliteal Depression & $52 \%$ \\
Medioposterior Tibial and & $52 \%$ \\
Femoral Condyles & \\
Lateroposterior Tibial and & $50 \%$ \\
Femoral Condyles & \\
(Posterior) Fibular Head & $49 \%$ \\
End of Fibia & $46 \%$ \\
Medial Tibial Condylar Flare & $37 \%$ \\
Hamstring Tendons & $37 \%$ \\
Shaft of Fibia & $34 \%$ \\
\hline Average Number of Socket & \\
Nodes Modified & \\
Standard Deviation in Number & \\
of Socket Nodes Modified & \\
\hline \hline
\end{tabular}

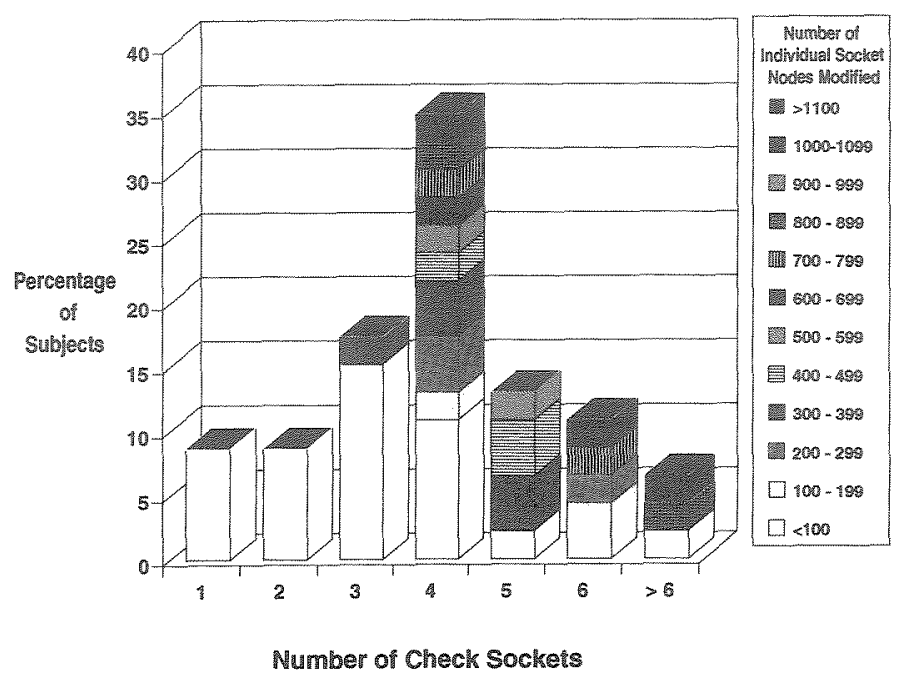

Tigure $35^{\circ}$

Number of check sockets and custom, individual socket node modifications required to achieve successful CASD/CAM socket designs and fittings for the AFMA test subjects.
Table 4.

Time-productivity relationship for design, production, and fitting of sockets and prostheses with the UCL-BC CASD/CAM System

\section{CASD/CAM PROSTIIESIS PRODUCTION TASRS}

AVERAGE
WORK-TIME
REQUIRED
PER TASK
(HOURS)

A. Patient interview, examination, measurement, residual limb casting.

B. Digitization of residual limb wrap cast. (May be done by technician.)

C. Design of CASD socket with five or fewer changes in UCL-BC modification template regions, and carving of positive socket model.

D. Designing of CASD socket with more than five changes in UCL-BC modification template regions, and carving of positive socket model.

E. Modification of individual socket node radii for smoothing and customization of socket design for individual patients.

$\mathrm{T}_{\mathrm{PN}}$ (in hours) $=$

Integer $\left[0.20^{*}\right.$ (Number socket nodes modified $\left.\left.)^{1 / 2}\right]\right\} / 4$

$T_{P N}$

F. Fabrication of thermoplastic check socket. (May be done by technician.)

G. Titting of check socket and recording of fitting data.

H. Fabrication of definitive socket, prosthetic component assembly, and bench alignment of CASD/CAM prosthesis prototype. (May be done by technician.)

1. Fitting and alignment of CASD/CAM prosthesis.

J. Cosmetic covering of final prosthesis. (May be done by technician.)

K. Delivery and evaluation of definitive CASD/CAM prosthesis.

the subjects, especially in the region of the anterodistal tibia when the socket had to be lengthened; 3) to extend and smooth the template modification region along the lateral tibial shaft to distribute residual limb/socket loading stresses more 
uniformly over the pretibial musculature, and help to prevent impingement of the anterodistal tibia during gait, for 59 percent of the subjects; 4) to extend distally and smooth the template modification region along the flare of the medial tibial shaft for 52 percent of the subjects in order to distribute soft tissue stresses more uniformly, and help to prevent impingement of the anterodistal tibia during ambulation; 5) to change the radii over the posterior aspect of the socket, in order to broaden and lengthen the popliteal depression for a more uniform distribution of weight bearing stresses for 52 percent of the subjects; 6 ) to reduce the socket radii over the medioproximal and medioposterior aspects of the socket encompassing the medial tibial and femoral condyles for 52 percent of the subjects, to achieve better conformity to anatomical contours, and to provide better mediolateral support; 7) to reduce the radii of the lateroproximal and lateroposterior aspects of the socket over the lateral tibial and femoral condyles to achieve better conformity to anatomical contours and better mediolateral support for 50 percent of the subjects; 8) to increase the magnitude, and/or extend posteriorly, the relief for the fibular head for 49 percent of the subjects; 9) to change the magnitude and/or move the location of the fibular end relief for 46 percent of the subjects; 10) to smooth and slightly decrease the socket radii over the medial tibial condylar flare, to increase socket/residual limb loading in this region for 37 percent of the subjects; 11) to change the posterior socket brim to provide additional relief for the hamstring tendons for 37 percent of the subjects; and, 12) to reduce the socket radii over the shaft of the fibia to achieve greater mediolateral support for 34 percent of the subjects.

Conditional analyses of the AFMA test data with respect to the subject conditions and characteristics affecting residual $\mathrm{limb} /$ socket loading were performed to determine if there were specific subsets of test subjects with common physiological/medical conditions, or with similar biomechanical and prosthetics characteristics requiring common UCL-BC CASD template and/or individual socket node modifications. Figure 36 shows the work-time required for successful CASD/CAM socket and prosthesis design, production, and fitting conditioned on the AFMA test subjects' cause of amputation. Figure 37 shows the work-time required as a function of the subjects' residual limb shape. Figure

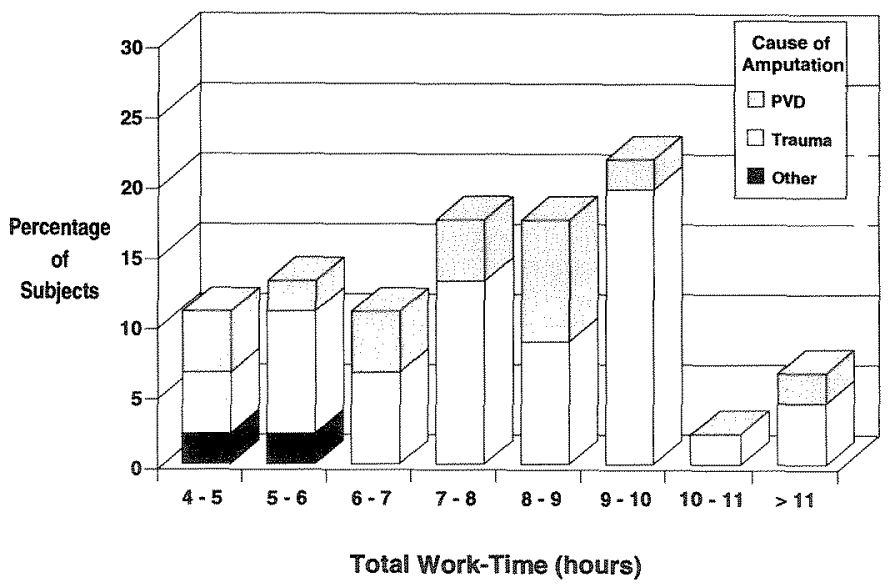

Figure 36.

Total work-time (personnel-hours) required for successful CASD/CAM socket and prosthesis design, production, and fitting as a function of subject cause of amputation.

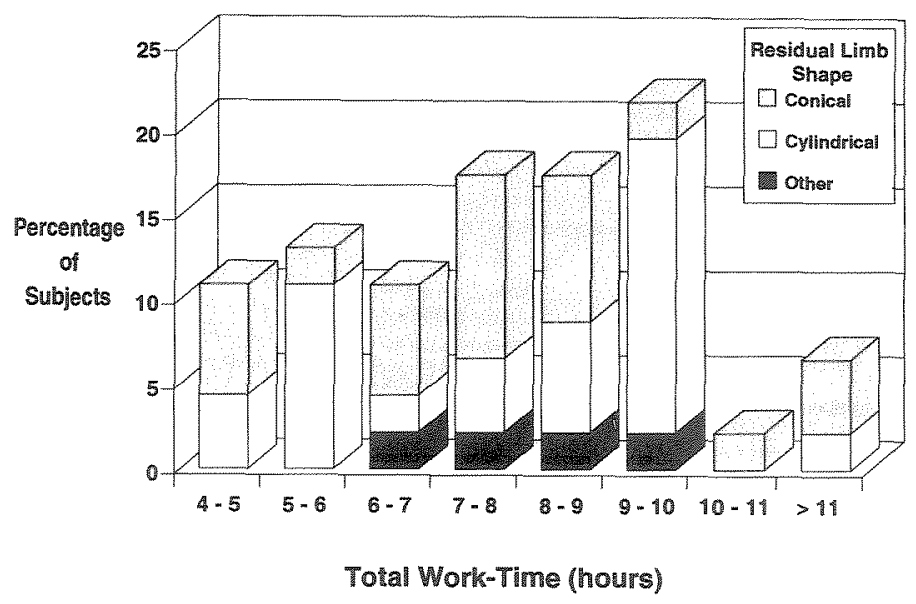

Pigure $3 \%$.

Total work-time (personnel-hours) required for successful CASD/CAM socket and prosthesis design, production, and fitting as a function of residual limb shape.

38 shows the work-time required as a function of the residual limb length of the subjects. Figure 39 shows the work-time required as a function of the subjects' type (relative durometer) of residual limb soft tissue. Figure 40 shows the work-time required as a function of the subjects' amount of residual limb soft tissue. And Figure 41 shows the work-time required for successful CASD/CAM socket and prosthesis design, production, and fitting as a function of the level of activity of the subjects (the percentage of the subjects' total daily prosthesis usage spent in load bearing activities, i.e., standing, 
Journal of Rehabilitation Research and Development Vol. 29 No. 4 Fall 1992

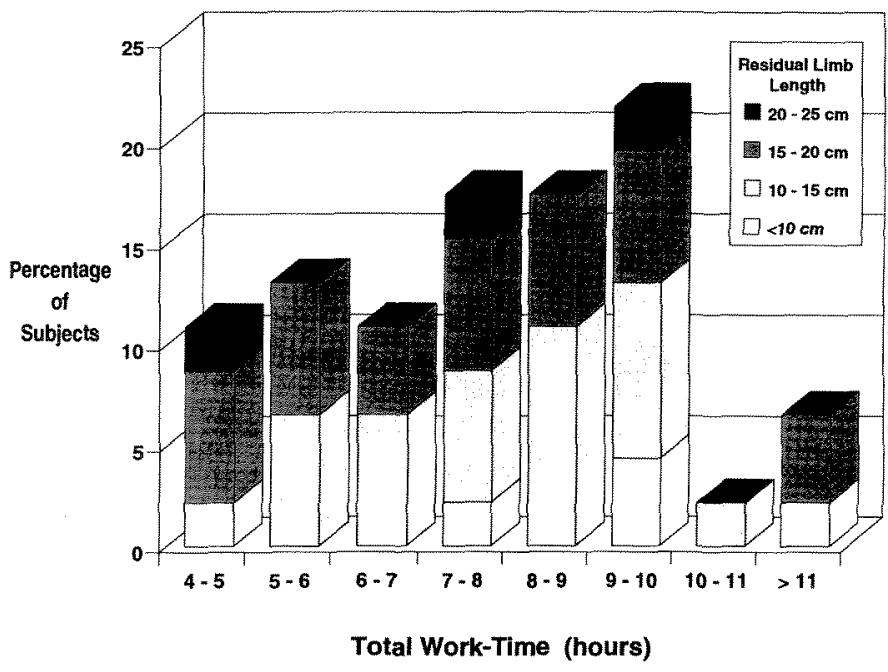

Figure 38.

Work-time (personnel-hours) required for successful CASD/CAM socket and prosthesis design, production, and fitting as a function of residual limb length.

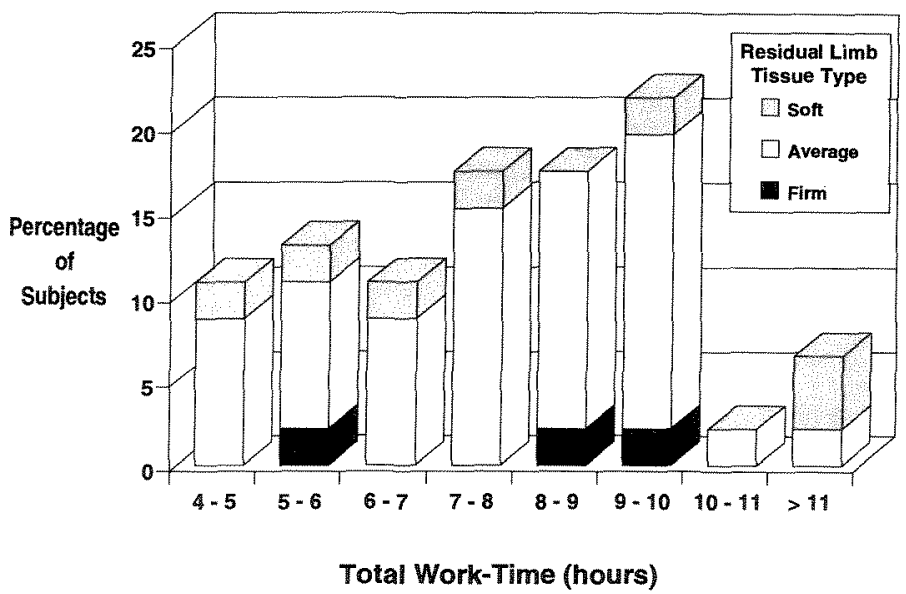

Figure 39.

Work-time (personnel-hours) required for successful CASD/CAM socket and prosthesis design, fitting, and production as a function of residual limb soft tissue type (relative durometer).

walking, and running). The average work-times required for successful CASD/CAM socket and prosthesis design, production, and fitting, together with their first sigma dispersions (average work-time plus and minus the sample standard deviation) are shown in Figures 42, 43, and 44, as a function of subject cause of amputation, residual limb shape, and residual limb length, respectively. To reduce the amount of dispersion in the resulting distributions,

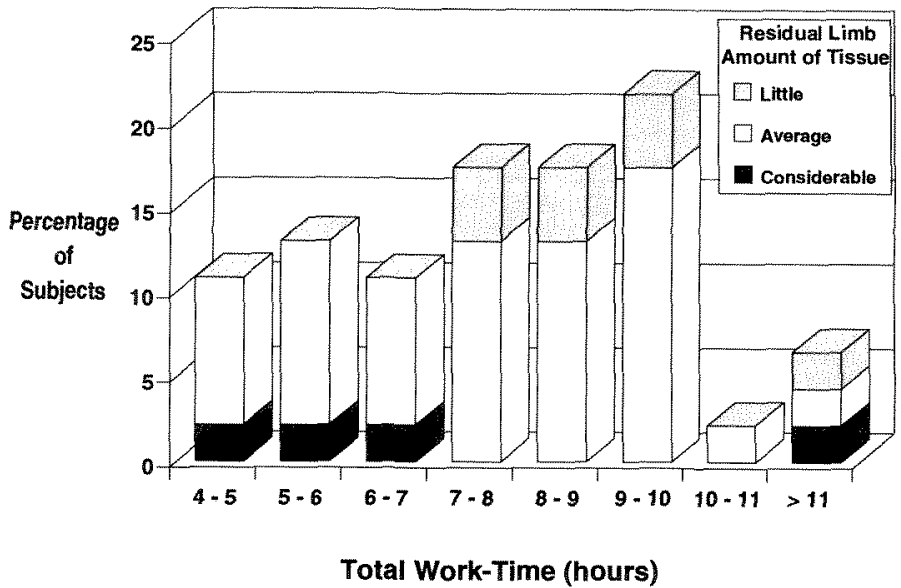

Figure 40.

Work-time (personnel-hours) required for successful CASD/CAM socket and prosthesis design, production, and fitting as a function of amount of residual limb soft tissue.

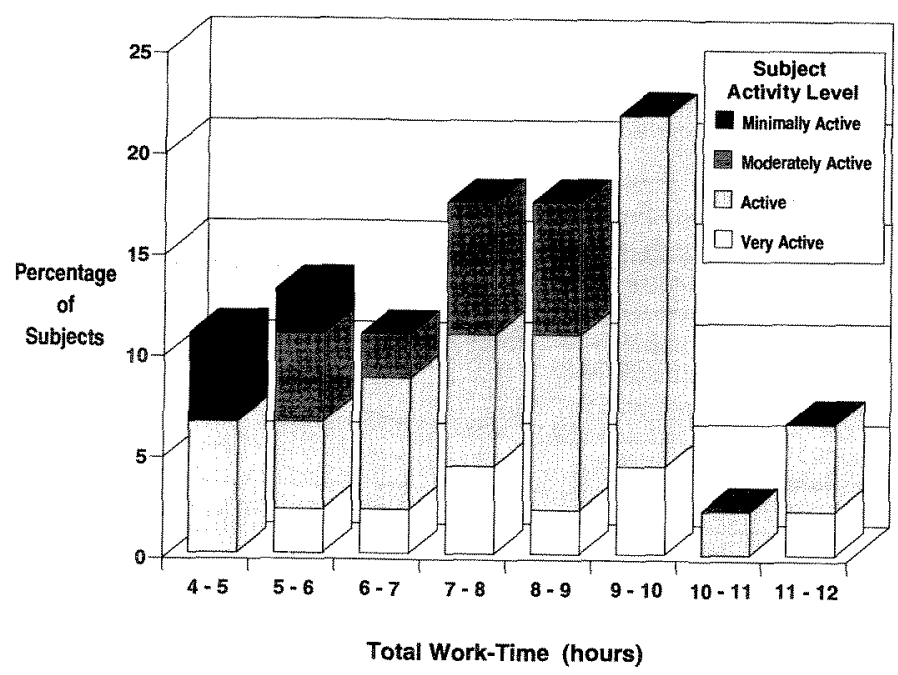

Figure 41.

Work-time (personnel-hours) required for successful CASD/CAM socket and prosthesis design, production, and fitting as a function of subject activity level.

the data were further conditioned on the AFMA Center where the subjects' CASD/CAM prostheses were designed, produced, and fitted. Conditioning the data by AFMA Center is believed to provide a more uniform measure among patient groups with respect to design, fitting, and evaluation criteria, prosthetist training and skill, etc. This is substantiated, in part, by considering the subjects' ratings of their prostheses conditioned on the AFMA Center 
where they were tested. On the zero-to-five rating scale used in the AFMA Study, the PRS test subjects gave their UCL-BC CASD/CAM prostheses an average overall satisfaction rating of 3.94 , with a sample standard deviation of \pm 0.86 ; an average rating of the quality of their UCL-BC CASD/CAM socket fit of 3.74 , with a sample standard deviation of \pm 1.03 ; and an average rating of the degree of their UCL-BC CASD/CAM socket comfort of 4.01, with a sample standard deviation of \pm 0.84 . The NU-VALMC test subjects gave their UCL-BC CASD/CAM prostheses an average overall satisfaction rating of 3.84 , with a sample standard deviation of \pm 0.93 ; an average rating of the quality of their UCL-BC CASD/CAM socket fit of 3.53 , with a sample standard deviation of \pm 1.28 ; and an average rating of the degree of their UCL-BC CASD/CAM socket comfort of 3.63 , with a sample standard deviation of \pm 1.03 . The NY DVAMC test subjects gave their UCL-BC CASD/CAM prostheses an average overall satisfaction rating of 3.88 , with a sample standard deviation of \pm 0.84 ; an average rating of the quality of their UCL-BC CASD/CAM socket fit of 3.75 , with a sample standard deviation of \pm 0.87 ; and an average rating of the degree of their AFMA socket comfort of 4.10 , with a sample standard deviation of \pm 0.74 . These results show that, although there were significant differences among the three AFMA Centers in the test subject sample populations, the level of training and experience of the AFMA prosthetists, the type and number of socket modifications used, etc., the results (rates of successful socket/prosthesis design and fitting) achieved at each of the AFMA Centers with the UCL-BC CASD/CAM system were very similar.

Figure 45 shows the average work-time required for successful UCL-BC CASD/CAM socket and prosthesis design, production, and fitting conditioned on the subjects' cause of amputation and the AFMA Center where the subjects were tested, together with the first sigma dispersions of the respective work-time distributions. The data are seen to be more consistent when conditioned on the AFMA Center where the subjects were tested. Also as seen in Figure 45, the differences in required work-times observed for the AFMA test subjects, as a function of cause of amputation, were not large enough to statistically differentiate between subsets of subjects. Specifically, since the average work-

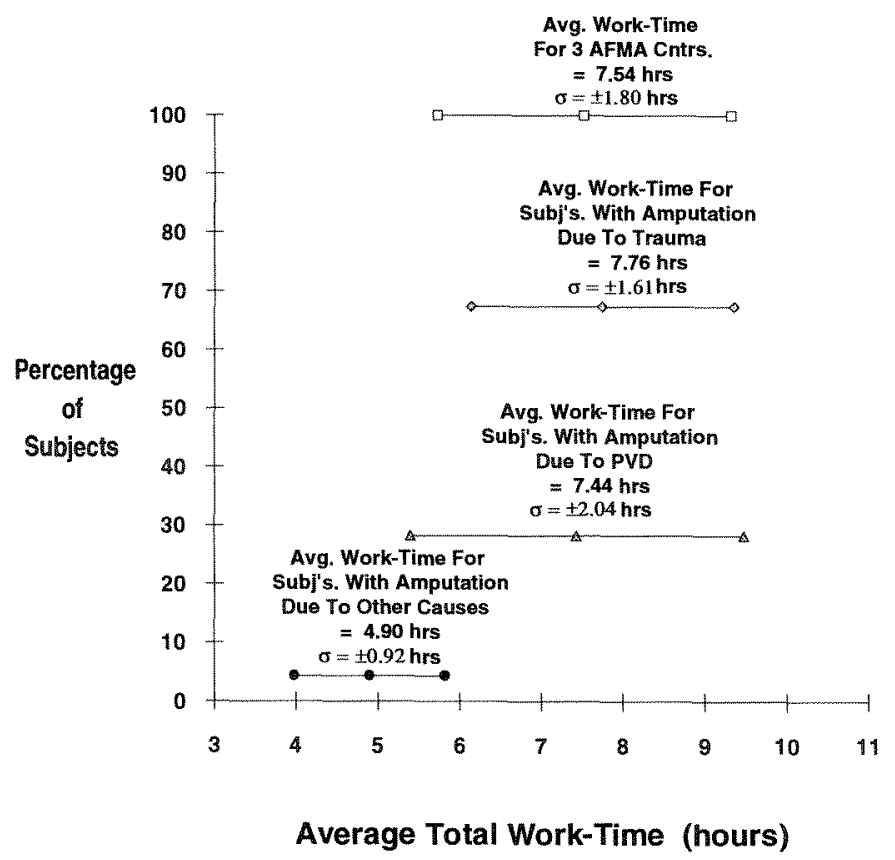

Figure 42.

Average total work-time (personnel-hours) required for successful CASD/CAM socket and prosthesis design, production, and fitting, with first sigma dispersion (plus and minus one standard deviation) of the work-time distributions, categorized by the subjects' cause of amputation.

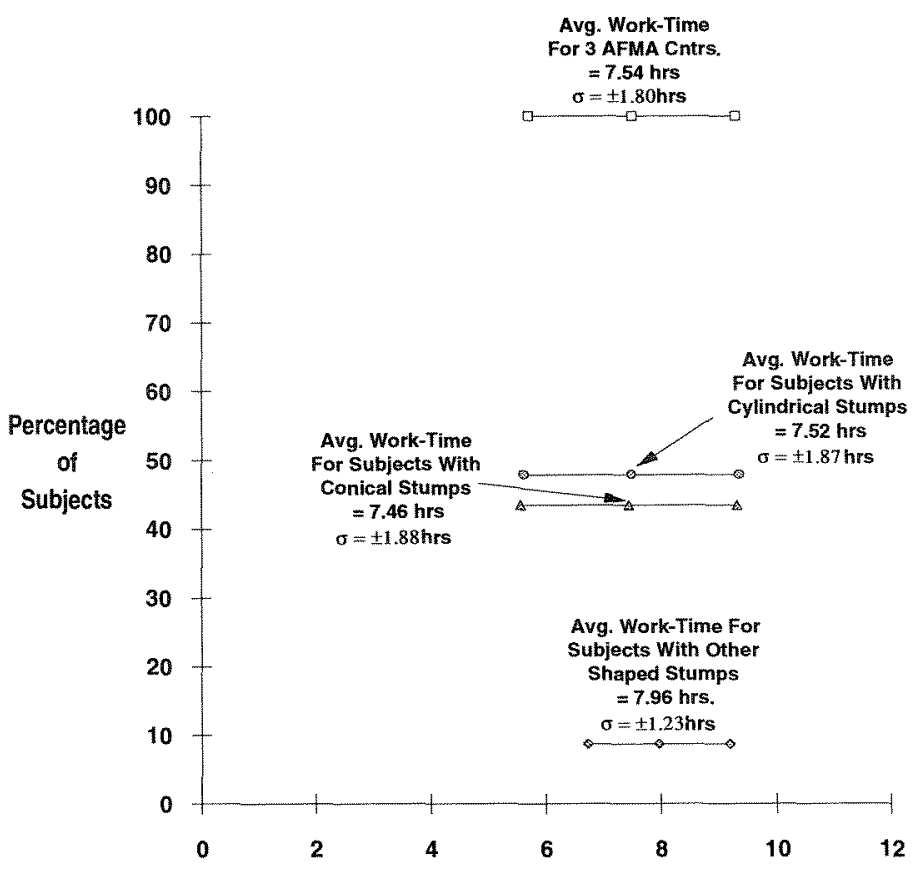

Average Total Work-Time (hours)

Figure 43.

Average work-time (personnel-hours) required for successful CASD/CAM socket and prosthesis design, production, and fitting as a function of residual limb shape, together with the first sigma dispersions of the work-time distributions. 
Journal of Rehabilitation Research and Development Vol. 29 No. 4 Fall 1992

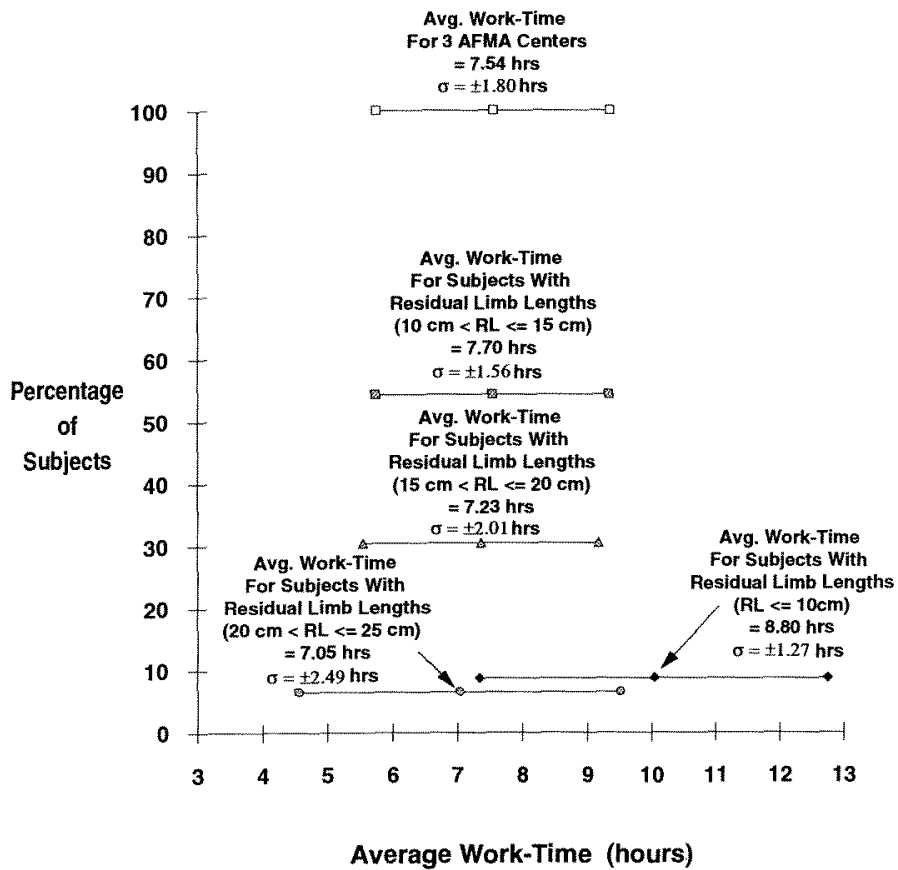

Figure 44.

Average work-time (personnel-hours) required for successful CASD/CAM socket and prosthesis design, production, and fitting as a function of residual limb length (RL), with first sigma dispersions of the work-time distributions.

times required for the categories of subjects by cause of amputation were approximately equal, and the first sigma dispersions of the respective work-time distributions overlapped, the groups were not statistically distinct enough to separate by cause of amputation. Some subjects with amputations due to PVD required less work-time and some required more work-time than subjects with amputations due to trauma, but on the average, the times required to design sockets for and successfully fit subjects in the two groups were approximately the same. The number of AFMA subjects tested with amputations due to other causes was too small to make statistical inferences with respect to work-time.

As seen in Figure 46, the work-time required for subjects with cylindrically shaped residual limbs was, on the average, approximately the same as the work-time required for subjects with conically shaped residual limbs. In addition, the first sigma dispersions of the distributions were approximately the same. Hence, the work-time requirements for the groups were very similar, and distinctions between the two groups based on first and second

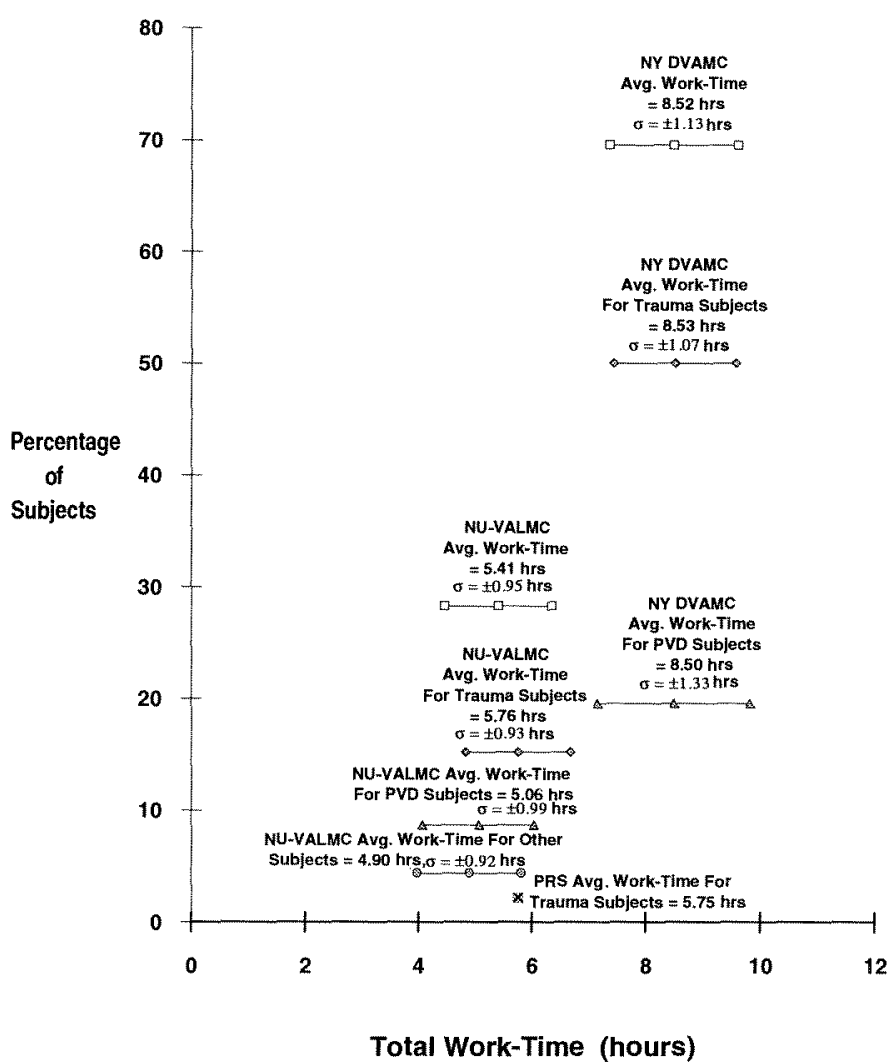

Figure 45.

Average total work-time (personnel-hours) required for successful CASD/CAM socket and prosthesis design, production, and fitting, categorized by subject cause of amputation, and AFMA Center, together with first sigma dispersions of the work-time distributions.

order statistics could not be made. The number of subjects tested with other shaped residual limbs was too small to make statistical inferences with respect to work-time.

As seen in Figure 47, the subjects who required the longest work-times to design sockets for and fit had the shortest residual limbs. In addition, the average work-time required for subjects with short residual limbs was greater than the average worktime required for subjects with either medium or long residual limbs. The first sigma dispersions of the respective work-time distributions, however, conditioned on subject residual limb length, overlapped, so not all subjects with short residual limbs required more time to design sockets for and fit than subjects with medium or long residual limbs. Hence, the hypothesis, "subjects with short residual limbs require more work-time to design sockets for and successfully fit with the UCL-BC CASD System 


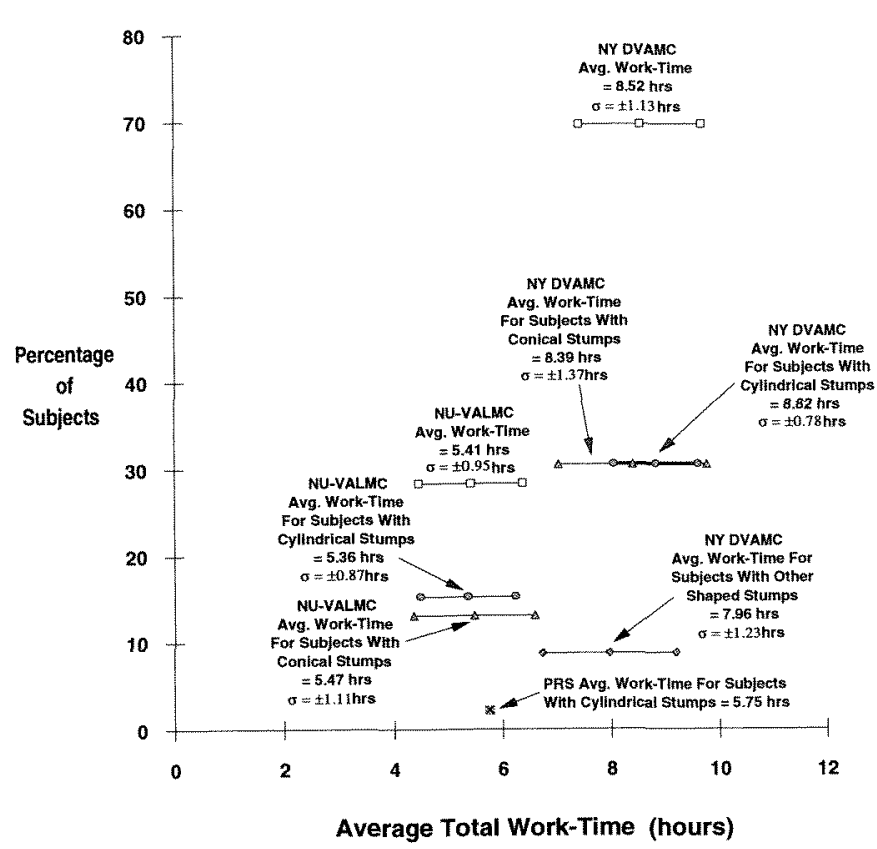

Figure 46.

Average work-time (personnel-hours) required for successful CASD/CAM socket and prosthesis design, production, and fitting, categorized by residual limb shape and AFMA Center, together with the first sigma dispersions of the work-time distributions.

than subjects with medium or long residual limbs," was only observed to be true 83 percent of the time for the AFMA test subjects. To further corroborate this, sample correlations between the residual limb length of the test subjects and the work-time required for successful socket design and fitting were calculated, assuming randomness and statistical independence in AFMA test subject selection. For the three AFMA Centers, a sample correlation coefficient of -0.136 was obtained. Further conditioning the data with respect to the AFMA Centers where the subjects were tested resulted in sample correlation coefficients of -0.388 and -0.237 for the NU-VALMC and NY DVAMC test subjects, respectively, thus indicating a mild trend toward increasing work-time with decreasing residual limb length. Restricting calculation to the subset of subjects with short residual limbs (i.e., those subjects with residual limb lengths less than or equal to 10 centimeters), however, resulted in an average sample correlation coefficient of -0.769 for the three centers, indicating a strong correlation between required work-time and decreasing residual

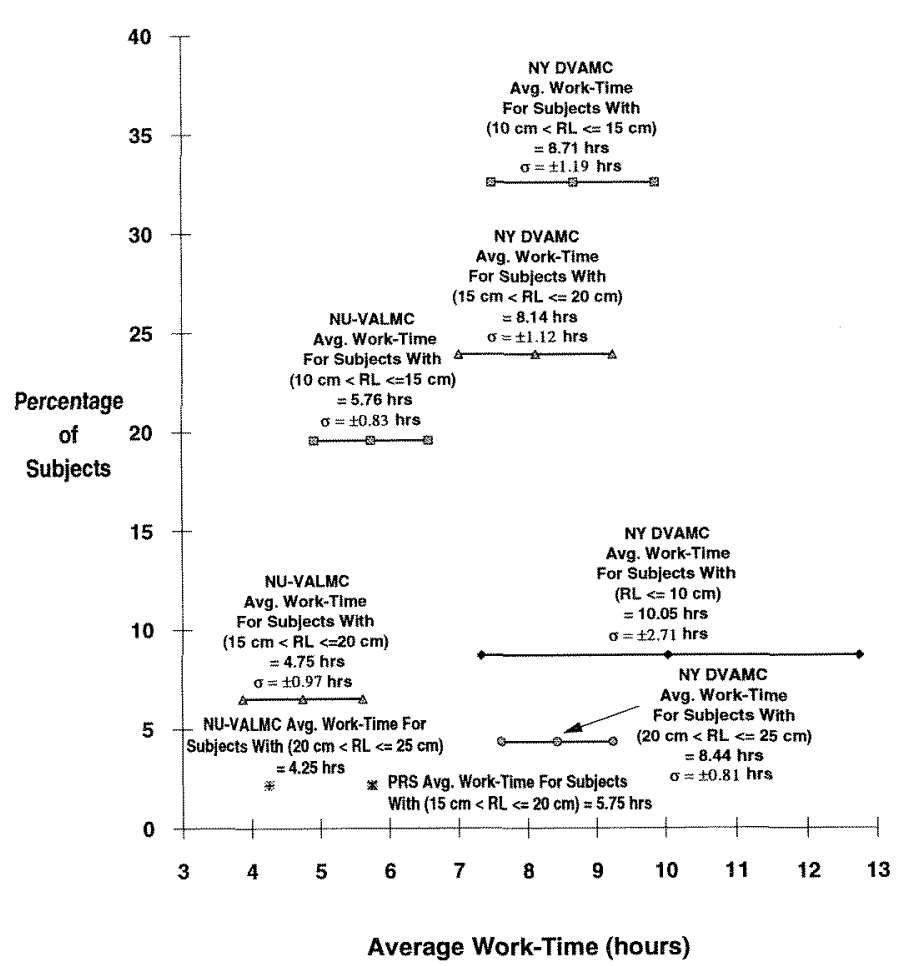

Figure 47.

Average work-time (personnel-hours) required for successful CASD/CAM socket and prosthesis design, production, and fitting categorized by residual limb length (RL) and AFMA Center, together with first sigma dispersions of the work-time distributions.

limb length for these subjects. Calculation of the sample correlations for the AFMA test subjects with medium-length residual limbs (those subjects with residual limb lengths between 20 and 50 percent of their contralateral limb length) and relatively long residual limbs (those subjects with residual limb lengths greater than 50 percent of their contralateral limb length) resulted in sample correlation coefficients of -0.112 and -0.069 , respectively, indicating weak to no correlation for these subjects. Hence, it follows from these results that the UCL-BC CASD system produced socket designs that, with limited additional modifications, were deemed very good to fair by a majority of the test subjects with medium and long residual limbs. Sockets designed for subjects with short residual limbs, however, required considerably more work, with a significant number of additional modifications, on the average, before acceptable socket designs and fits were achieved. Hence, development of new CASD socket design templates, affording a more accurate and 


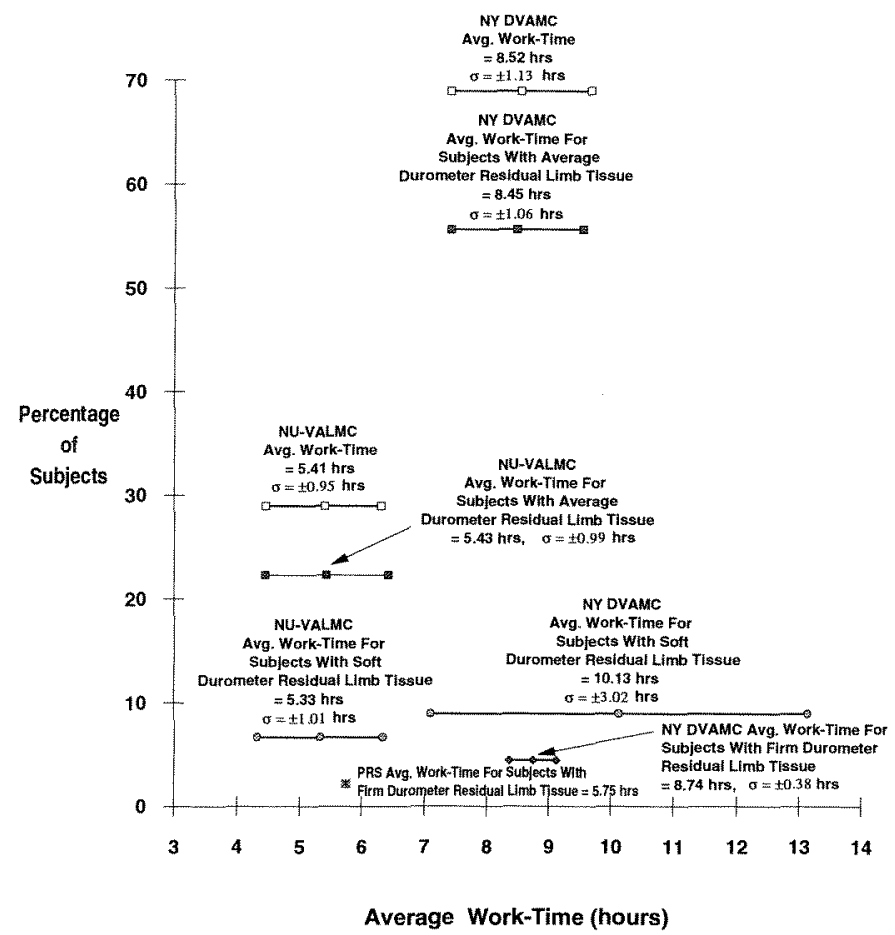

Figure 48.

Average work-time (personnel-hours) required for successful CASD/CAM socket and prosthesis design, production, and fitting categorized by residual limb soft tissue type (relative durometer), and AFMA Center, together with first sigma dispersions of the work-time distributions.

intimate initial fit for subjects with short residual limbs, is indicated.

As seen in Figure 48, the average work-time required for subjects with firm durometer residual limb tissues was slightly larger than the average work-time required for subjects with average durometer residual limb tissues. The first sigma dispersion of the work-time distribution for subjects with average durometer residual limb tissues was, however, slightly greater than that for the subjects with firm durometer residual limb tissues, so some subjects with average durometer residual limb tissues required more work to design sockets for and fit than subjects with firm durometer tissues. The work-time required for subjects with soft durometer residual limb tissues was, on the average, greater than that for subjects with either firm or average durometer tissues. In addition, Figure 48 shows that the first sigma dispersion of the work-time distribution for subjects with soft durometer tissues was greater than, and overlapped, the first sigma dispersions of the work-time for subjects with firm and with average durometer residual limb tissues. Therefore, not all subjects with soft residual limb tissues required more work to design sockets for and to fit successfully than subjects with average or firm durometer tissues. Calculation of sample correlation coefficients between the work-time required for successful CASD/CAM socket design and fitting and subject residual limb tissue durometer, indicated weak to no correlation between the required worktimes and residual limb tissue type for subjects with average and firm durometer tissues. A slight negative correlation, -0.113 , however, was obtained for subjects with soft and very soft residual limb tissues. Hence, for the 11 percent of the test subjects with soft and very soft durometer residual limb tissues, as the tissue durometer decreased, the amount of work-time required to achieve successful socket designs and fits tended, on the average, to increase. The number of test subjects with firm and very soft residual limb tissues was relatively small, however, so further testing should be conducted to confirm these results.

Figure 49 shows that the work-time required for subjects with a considerable amount of residual limb soft tissue was, on the average, slightly greater than that required for subjects with an average amount of residual limb soft tissue. The work-time required for subjects with little residual limb soft tissue was, on the average, significantly greater than that required for either subjects with considerable or average amounts of residual limb tissue. The first sigma dispersions of the respective distributions overlapped, however, so differentiation between categories of subjects as a function of the amount of their residual limb soft tissue, by the work-time they required for successful CASD/CAM socket design and fitting, was not possible. Calculation of the relative frequency of occurrence of the trend toward increasing work-times with variation from an average amount of residual limb tissue, shows that 55 percent of the subjects with a considerable amount of residual limb tissue required more work to successfully design sockets for and fit than subjects with an average amount of tissue. Eighty-seven percent of the subjects with little residual limb soft tissue required work-times greater than those for subjects with either average or considerable amounts of residual limb soft tissues. Calculation of sample 
correlation coefficients between required work-times and residual limb tissue volume evidenced only slight correlation, 0.218 , with an increase in the work-time required with increasing amount of residual limb soft tissue for those subjects with a considerable amount of tissue, and -0.288 for those subjects with little residual limb soft tissue, thus indicating an increase in the work-time required with decreasing tissue volume for these subjects. The number of AFMA test subjects with little and with considerable amounts of residual limb soft tissues was smaller than the number of subjects with an average amount of residual limb soft tissues, so further clinical testing should be conducted to verify these trends.

The relationship between subject activity level and the work-time required for successful socket design and fitting was also investigated. As a measure of activity level, the percentage of the average time per day the test subjects wore their prostheses and were engaged in load-bearing activities (i.e., standing, walking, or running) was calculated. From these results, four categories of activity level were defined: 1) minimally active-for those subjects whose percentage of active prosthesis use was less than 25 percent of their total average daily prosthesis usage; 2) moderately active-for those subjects whose percentage of active prosthesis use was greater than or equal to 25 percent, but less than 50 percent, of their total average daily prosthesis usage; 3) active-for those subjects whose percentage of active prosthesis use was greater than or equal to 50 percent, but less than 75 percent, of their total average daily prosthesis usage; and, 4) very active-for those subjects whose percentage of active prosthesis use was greater than or equal to 75 percent of their total average daily prosthesis usage. As none of the test subjects reported prosthesis usage patterns with relatively short periods of intense usage, and otherwise long periods of relatively inactive usage, these classifications are believed to characterize the AFMA test subjects adequately. Conditioning the data with respect to these categories, a monotonic increase in the average required work-time with increasing activity category was observed (Figure 50). The first sigma dispersions of the work-time distributions for the respective activity level categories overlapped, however, indicating this trend did not hold for all subjects. Statistically, it was found that: 1) 92 percent of the

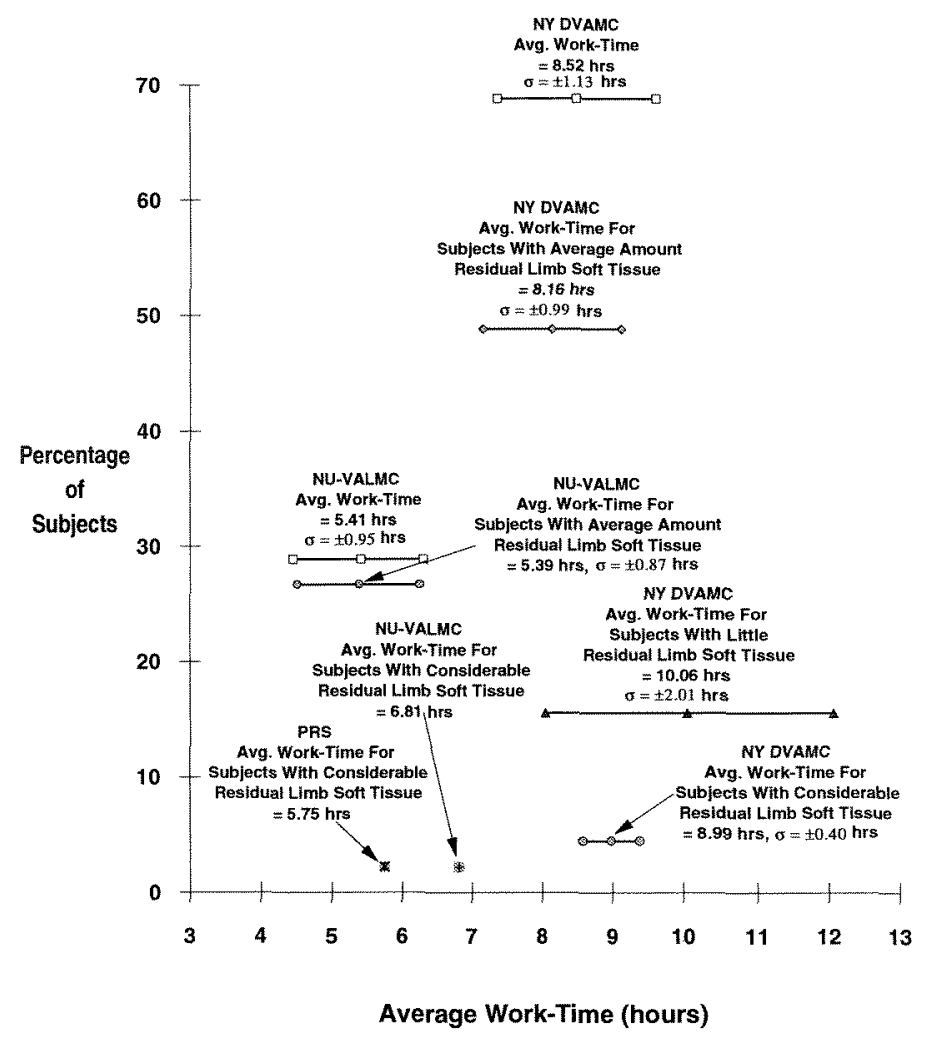

Figure 49.

Average work-time (personnel-hours) required for successful CASD/CAM socket and prosthesis design, production, and fitting categorized by amount of residual limb soft tissue and AFMA Center, with first sigma dispersions of the work-time distributions.

moderately active subjects required more work to design sockets for and to fit successfully than the minimally active subjects; 2) 72 percent of the active subjects required more work to design sockets for and to fit than the moderately active and the minimally active subjects; and, 3) 63 percent of the very active subjects required more work to design sockets for and to fit than the active, the moderately active, and the minimally active subjects. Calculation of the sample correlation coefficients between subject activity level and required work-time resulted in values of $-0.0467,0.101,0.353$, and 0.497 , respectively, for the four activity level categories, also indicating a trend toward increasing work-time with increasing activity level. Hence, in the majority of cases, the more the AFMA test subjects actively used their prostheses, the more intimate and accurate prosthetic socket fits they required (and demanded), and the more work that 


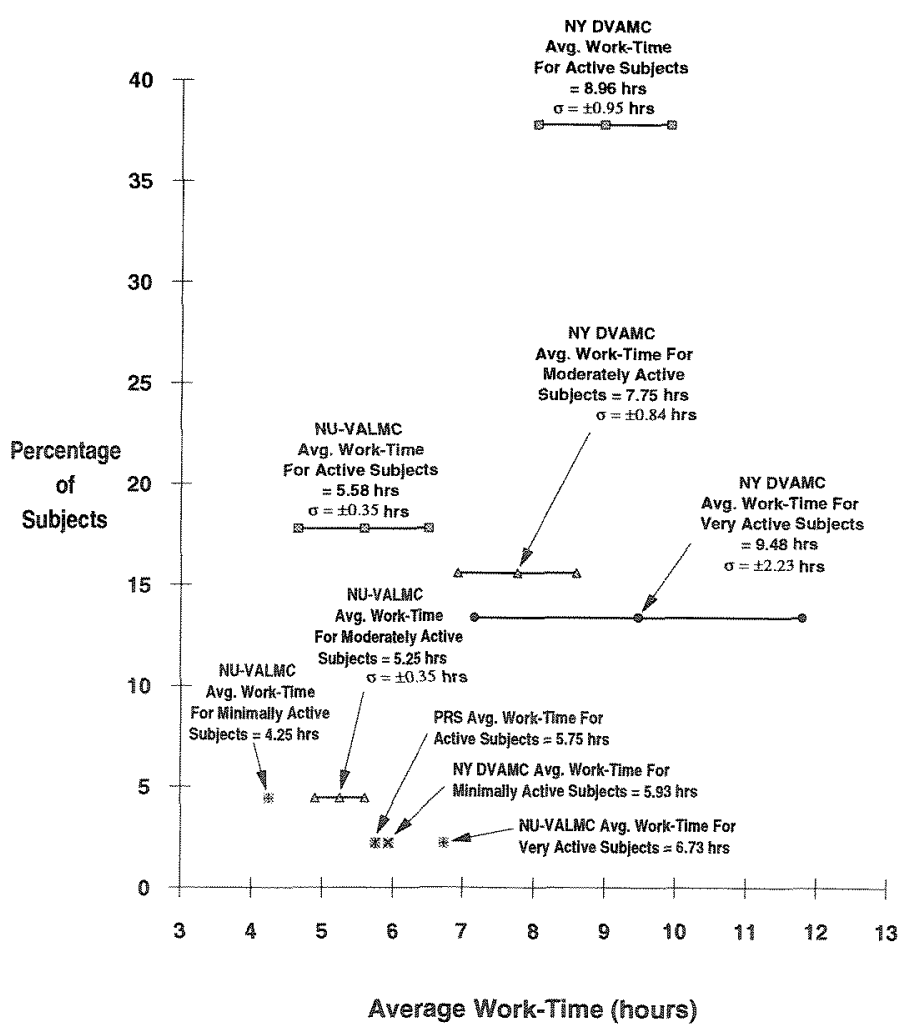

Figure 50.

Average work-time (personnel-hours) required for successful CASD/CAM socket and prosthesis design, production, and fitting categorized by subject activity level and AFMA Center, with first sigma dispersions of the work-time distributions.

had to be expended in modification of the initial UCL-BC socket design, before acceptable, wellfitting, comfortable CASD/CAM sockets were obtained. These results indicate that the UCL-BC CASD socket templates should be refined further to provide socket designs more intimately and accurately matched to subjects' residual limbs. This would not only afford improved CASD socket designs and fits for a majority of patients, but should also reduce the amount of socket redesign work required, and hence, should considerably increase the productivity achieved.

Consideration of the other parameters affecting residual limb/socket loading, and hence, socket design, either resulted in categories with too few subjects to yield statistically meaningful results, or failed to evidence any significant correlation. Further analysis, therefore, was restricted to the subset of subjects who required the most work to design sockets for and to fit successfully. The most difficult to fit subset of the AFMA test subjects (those requiring more than the average work-time plus one-half a standard deviation) was compiled and analyzed for common, distinguishing characteristics (Figure 51). Three of the subjects in the difficult to fit group had medical/physiological complications that made them difficult to fit using either CASD/CAM techniques or conventional prosthetics techniques. Because of the medical/physiological complications, these three unusually difficult to fit subjects required more work (i.e., a greater number of socket design modifications and subsequent clinical check sockets) than any of the other difficult to fit or non-difficult to fit test subjects. Despite this, after considerable work, successful CASD/CAM sockets were able to be designed for and fit to these subjects, further demonstrating the range of the UCL-BC CASD/CAM system's capabilities. The other subjects in the difficult to fit group evidenced appreciable statistical differences from the nondifficult to fit subjects with respect to residual limb length and subject activity level. With respect to residual limb length, 16 percent of the difficult to fit subjects had short residual limbs, 79 percent had average-length residual limbs, and 5 percent had long residual limbs. The average work-time for the difficult to fit subjects with short residual limbs was 10.93 hours, for the difficult to fit subjects with average-length residual limbs 9.75 hours, and for the difficult to fit subjects with long residual limbs 9.01 hours. Comparatively, 4 percent of the non-difficult to fit subjects had short residual limbs, 89 percent had average-length residual limbs, and 7 percent had long residual limbs. The average work-time for the non-difficult to fit subjects with short residual limbs was 7.42 hours, for the non-difficult to fit subjects with average-length residual limbs 6.40 hours, and for the non-difficult to fit subjects with long residual limbs 6.06 hours. With respect to activity level, 4 percent of the difficult to fit subjects were minimally active, 15 percent were moderately active, 58 percent were active, and 23 percent were very active. The average work-time for the minimally active, difficult to fit subjects was 9.02 hours, for the moderately active, difficult to fit subjects 9.01 hours, for the active, difficult to fit subjects 9.38 hours, and for the very active, difficult to fit subjects 11.15 hours. For the non-difficult to fit subjects, 4 percent were minimally active, 22 percent were moderately active, 56 percent were active, and 
18 percent were very active. The average work-time for the minimally active, non-difficult to fit subjects was 4.88 hours, for the moderately active, nondifficult to fit subjects 6.29 hours, for the active, non-difficult to fit subjects 6.45 hours, and for the very active, non-difficult to fit subjects 6.53 hours. The difficult to fit subjects also evidenced mild to slight statistical differences from the non-difficult to fit subjects with respect to subject weight, subject residual limb shape, amount and type of residual limb soft tissue, and prosthesis alignment. These latter differences, however, were not prevalent enough for the AFMA test subjects to be statistically significant.

Further analysis of the data revealed that of the implicit characteristics affecting socket/residual limb loading, the only characteristics that differed appreciably between the difficult and non-difficult to fit subjects were the subjects' time since amputation and the subjects' prosthesis satisfaction ratings. Specifically, the difficult to fit subjects averaged 36.2 years since the date of their amputation, with a standard deviation of 18.2 years, and a median of 44.0 years. Seventy-four percent of the difficult to fit subjects had amputation 20 years ago or more, and 63 percent had amputation 30 years ago or more. By comparison, the non-difficult to fit subjects averaged 18.8 years since the date of their amputation, with a standard deviation of 19.4 years, and a median of 18.0 years. Forty-one percent of the non-difficult to fit subjects had amputation 20 years ago or more, and 26 percent had amputation 30 years ago or more. Statistically, the difficult to fit subjects were amputees longer than the non-difficult to fit subjects with a relative frequency of occurrence of 68.4 percent (Figure 52). Calculation of the sample correlation coefficients between the worktime required for successful CASD socket design and fitting, and time since subject amputation yielded values of 0.38 and 0.36 for the difficult to fit and the non-difficult to fit subjects, respectively, indicating approximately the same degree of correlation between increasing work requirements and increasing time since amputation for the two groups.

On the zero-to-five rating scale used in the AFMA study, the difficult to fit subjects gave their pre-AFMA prostheses: $(a)$ an average overall satisfaction rating of 4.40 , with a standard deviation of 0.92 ; (b) an average quality of fit rating of 4.36 , with a standard deviation of 1.31 ; and, $(c)$ an

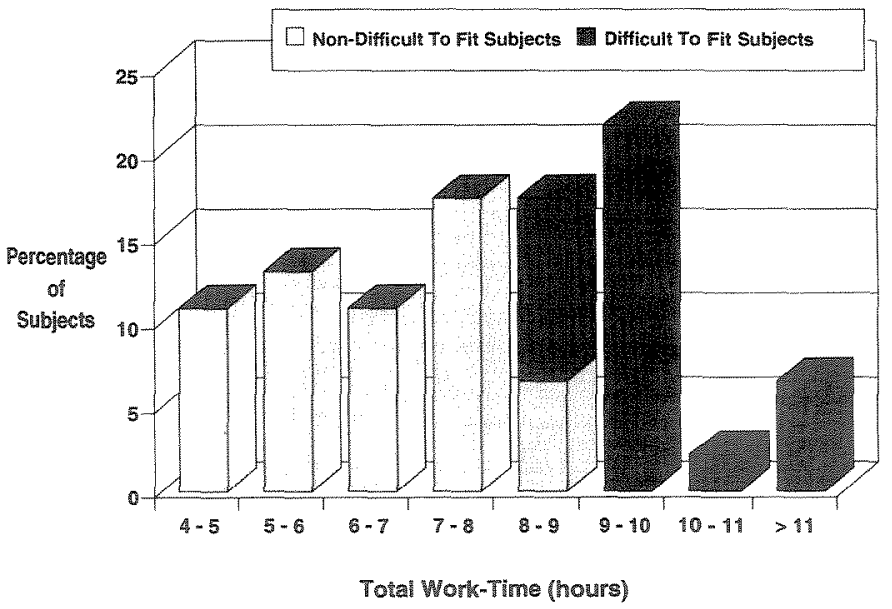

Tigure 51 .

Work-time (personnel-hours) required for successful CASD/CAM socket and prosthesis design, production, and fitting for the AFMA test subjects. The subjects requiring work-times greater than or equal to the average plus one-half a standard deviation were classified as "difficult to fit" with the UCL-BC CASD/CAM System.

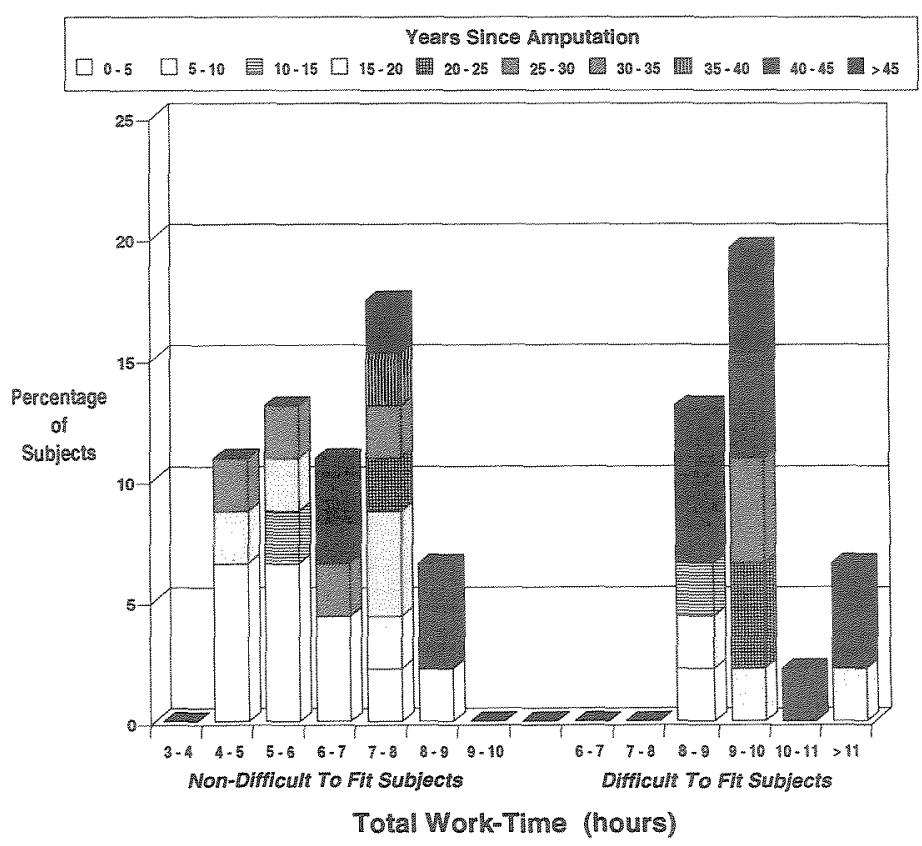

Figure 52.

Work-time (personnel-hours) required for successiul CASD/ CAM socket and prosthesis design, production, and fitting for the AFMA test subjects, categorized by the subjects" degree of difficulty (amount of work required) in fitting and the number of years since amputation. 
Joumal of Rehabilitation Research and Development Vol. 29 No. 4 Fall 1992

average comfort rating of 4.41 , with a standard deviation of 0.90 . The non-difficult to fit subjects, however, gave their pre-AFMA prostheses: $\left(a^{\prime}\right)$ an average overall satisfaction rating of 3.11 , with a standard deviation of $1.51 ;\left(b^{\prime}\right)$ an average quality of fit rating of 3.03 , with a standard deviation of 1.63 ; and, $\left(c^{\prime}\right)$ an average comfort rating of 3.16 , with a standard deviation of 1.15 (Figure $\mathbf{5 3}$ ). In addition, the difficult to fit subjects wore their pre-AFMA prostheses an average of 56.2 months before replacing them, and the non-difficult to fit subjects wore their pre-AFMA prostheses an average of 44.0 months before obtaining new prostheses.

Thus, excluding the three subjects with medical/ physiological complications, the difficult to fit subjects were generally longer-term amputees who were considerably more satisfied with their present, preAFMA prostheses than were the non-difficult to fit subjects. As such, the difficult to fit subjects were typically quite critical, and demanded that the fit, comfort, function, weight, and cosmesis at least equal the level of quality in their pre-AFMA prostheses before they deemed their new CASD/ CAM sockets and prostheses acceptable. Because of this, and the larger percentage of difficult to fit subjects with short residual limbs and higher activity levels, an average of 9.9 hours was required to achieve acceptable socket designs and fits for the difficult to fit subjects. Specifically, the difficult to fit subjects required an average of 5.7 check sockets, in which an average of 5.3 template modification regions and an average of 567 individual socket nodes had to be modified before successful socket designs and fits were achieved with the UCL-BC CASD/CAM system. Comparatively, the non-difficult to fit subjects required an average of 3.0 check sockets with an average of 4.6 template region and 86 individual socket node modifications, taking an average of 6.42 hours of work-time to successfully complete. The additional modifications and subsequent check sockets required for the difficult to fit subjects took, on the average, 54 percent more work-time than was required for the non-difficult to fit subjects. Hence, development of new, UCL-BC CASD socket design templates affording initial socket designs more intimately and accurately matched to individual subjects residual limbs is strongly indicated. Ironically, the class of difficult to fit subjects are ideal candidates for CASD/CAM sockets and prostheses. They are usually relatively.

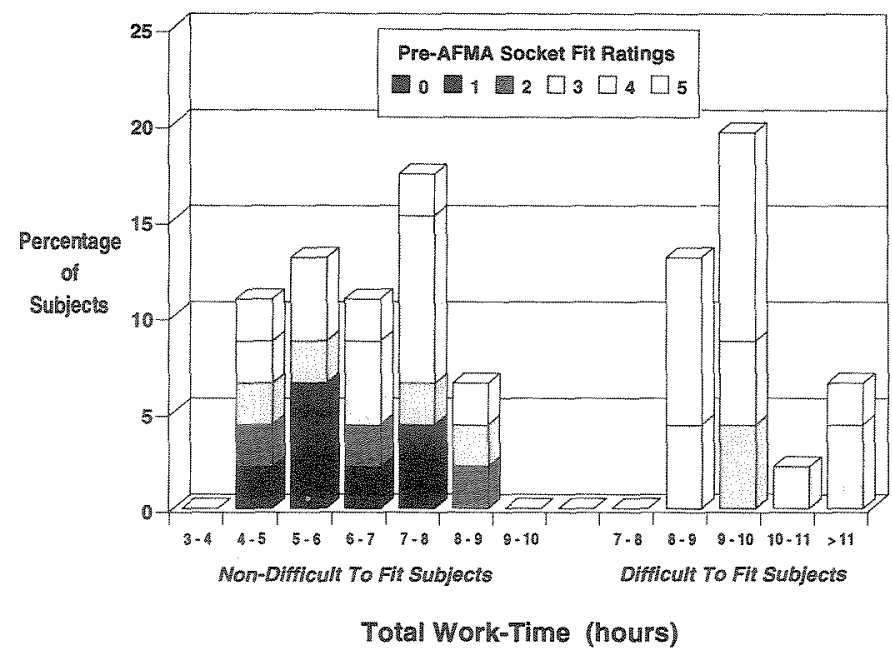

Figure 53 .

Work-time (personnel-hours) required for successful CASD/ CAM socket and prosthesis design, production, and fitting for the non-difficult to fit and the difficult to fit AFMA test subjects as a function of the subjects' ratings of the quality of fit of their conventional, pre-AFMA prostheses.

stable (i.e., their residual limb volume, tissue type, prosthesis fit and functional requirements, muscle strength and endurance, activity level, etc., are relatively constant), so that once successful socket and prosthesis designs and fits have been obtained and are on (computer) file, either by digitization of a favorite, existing socket, or through an initial, albeit more lengthy, CASD design and fitting process, production of another, new socket and prosthesis can be accomplished far more accurately, efficiently, and expediently using CAD/CAM technologies, than with conventional prosthetics procedures.

In summary, the clinical testing and evaluation of the UCL-BC CASD/CAM system conducted under the National AFMA Program showed it to be an intuitive, well-organized, relatively easy to use CASD/CAM system. Nevertheless, the relatively large number of template modification region foci that required change, the relatively large number of individual socket node radii that required modification, and as a consequence, the large number of check sockets required to achieve successful socket designs and fits indicate the productivity of the UCL-BC CASD/CAM system should be improved so its full potential as a truly effective and productive clinical prosthetics tool can be realized. The 
relatively large number of modifications and worktimes required to achieve successful CASD/CAM socket designs are felt to reflect more on the fit afforded by the socket templates tested and on certain strictures and operational limitations in the version of the UCL-BC CASD/CAM system tested, than to indicate fundamental limitations in the design philosophy underlying the UCL-BC system. The fact that only slight to mild correlations were found between the type, amount, and number of modifications and work required, and the subject characteristics explicitly related to residual limb/socket loading, indicates that the current UCL-BC CASD design templates need further refinement, and new, improved, templates based on a statistically representative and significant number of subjects need to be developed. Also, from their laboratory and clinical tests and fittings with the system, the AFMA investigators recommend that the UCL-BC CASD system be refined further to include: 1) the capability to accurately and automatically transfer multiple landmarks from the residual limbs of patients to digitized CASD measurement models; 2) the capability to automatically link, scale, and apply standard template modifications to regions identified and accurately located by specific residual limb landmarks included as part of patients' measurement files; 3) the capability for the prosthetist to adjust the size, shape, location, and topography of all modification regions; 4) the capability to define rapidly and accurately new modification regions of arbitrary size, shape, location, and topography for accommodation of pressure-sensitive regions (e.g., adherent scars, neuromas, cysts, bone spurs, etc.), and/or for increasing loading in stress-tolerant areas; and, 5) the capability to compute and apply all net resultant modifications at socket nodes so as to ensure linearity of sequential design iterations, and to ensure smooth transitions at all modification region boundaries. Incorporation of these capabilities should reduce the amount of work required to design sockets, and at the same time improve the ease and speed of use of the CASD system.

\section{UBC-MERU CASD System test results}

As the final part of the AFMA clinical testing, the UBC-MERU Canfit CASD System was tested on 10 subjects at the NY DVAMC and on 5 subjects at NU-VALMC. Unlike the UCL-BC CASD system that derives initial socket designs by superimposing modification templates on digitized measurements from casts of patients' residual limbs, the UBC-MERU Canfit System produced socket designs by matching, as best as possible, scaled, predefined anthropomorphic shapes to a set of residual limb measurements manually taken by the prosthetist. Regions of the resulting initial socket model that required additional contour modification because of: 1) residual limb geometries that did not conform to the predefined, anthropomorphic, socket shapes; 2) residual limb tissue anomalies with low stress tolerance, such as neuromas, bone spurs, cysts, etc.; or, 3) increased loading requirements over stress-tolerant residual limb regions (e.g., to offset stress reductions in other areas); had to be identified on the initial Canfit socket model and then appropriately modified by the prosthetist using CASD graphical shape manipulation functions. When the additional rectifications required by a given subject were completed, the resulting socket model was output to a computer file. This file was then processed by a format translation program and sent to the AFMA Center's UCL-BC CAM equipment for manufacture, because the UBC-MERU Canfit System did not include a carver or an NC vacuum thermoforming machine. Positive socket models were manufactured and subsequently fit on the test subjects as in the UCL-BC CASD/CAM system tests.

The NY DVAMC test subjects fit with UBC-MERU Canfit sockets required an average of 4.8 check sockets before acceptable socket designs and fits were obtained. The NU-VALMC test subjects required an average of 2.5 check sockets before acceptable socket designs and fits were achieved. Seventy-three percent of the subjects tested rated the fit of their Canfit sockets good to fair, and 27 percent as fair to poor. None of the subjects rated the fit of their Canfit sockets as very poor. The sockets produced with the Canfit system did not receive as high a satisfaction rating by as many subjects as did the best pre-AFMA prostheses, but more of the subjects rated their Canfit CASD sockets good to fair than rated their conventional pre-AFMA sockets good to fair. Hence, the UBC-MERU Canfit System did not produce socket designs that were rated as good by as many test subjects as the very best pre-AFMA sockets designed and manufactured using conventional prosthetics 
techniques. However, a greater number of sockets rated good to fair were designed and produced for the test subjects with the Canfit System than by the pre-AFMA prosthetists using conventional techniques. Furthermore, the worst sockets produced with the Canfit System were not rated as bad as the worst pre-AFMA sockets produced by some preAFMA prosthetists with conventional procedures.

During the clinical trials of the UBC-MERU CASD system, several problems were identified in the Canfit software. These errors together with general comments and recommendations for improvements in the Canfit System were given to the developers. In the comments/recommendations of the AFMA investigators, it was noted that the biflanged, tibial angulation/circumferential measurement caliper (Figure 6) used to characterize the transverse angulation of patients' tibias and to measure residual limb circumferences for Canfit System input had limited accuracy, relatively large measurement tolerances, and limited repeatability. Questions were raised regarding the completeness of the set of measurements used by the Canfit CASD System for residual limb characterization (anteroposterior and mediolateral diameters at the medial tibial plateau and the femoral condylar levels, respectively, residual limb length, and residual limb circumferences at $2.5 \mathrm{~cm}$ axial intervals). The menus and patient directory structure of the Canfit System were felt to be somewhat nonintuitive and cumbersome, especially with respect to some of the nonprosthetics terminology used. As such, the Canfit System took slightly longer to learn and become proficient with than the UCL-BC System. The fixed, single-sided, horizontal presentation of the Canfit socket model was also found to hinder some prosthetists' visualization of the CASD socket model. Otherwise, the graphical wireframe, shaded solid, and cross-sectional presentations of the socket model were deemed good, although rather small. The inability to pan and zoom in the wireframe and shaded solid models made identification of the location and the extent of specific residual limb regions difficult. The cache of shape modification methods contained in the Canfit System was deemed very good (socket length modification-both total and incremental; socket volume modification in increments of prosthetic ply; and ability to create custom modification regions with control of region foci magnitudes and topographies). The ability to move the posterior popliteal socket brim was useful for subjects with short and with long residual limbs. The ability to change socket volume proved to be very useful. It would have been even more so, however, if incremental volume changes over specified residual limb regions would have also been permitted, especially for newer amputees who undergo greater distal than proximal residual limb shrinkage. The inability to define multiple foci and their respective magnitudes for precise control of modification region topographies was deemed a disadvantage. Nonsmooth boundaries arose frequently using Canfit's single modification region creation function, because of its single focus. More precise control of modification region topographies and smoother boundaries could have been achieved, if it would have been possible to define multiple foci in modification regions and independently adjust their magnitudes. The socket "end cap" feature was also felt to need considerable refinement, as the cap added to the distal end of the socket models failed to blend smoothly with the adjoining socket contours, except for nearly cylindrical residual limb socket shapes.

With elimination of the remaining system bugs, and addition of the features and capabilities noted above, it is felt that the Canfit System could be an effective prosthetics design tool. A basic question remains, however, regarding the efficacy of the design philosophy underlying the UBC-MERU Canfit System. Use of predefined anthropometric shapes with postdesign modification and tailoring to accommodate individual requirements has proven quite effective in some applications. The tolerances required to successfully fit most amputees may be too small, however, to permit acceptable matches with predefined anthropomorphic shapes, without considerable further custom modifications. In the clinical trials of the Canfit System, the proximal socket from the tibial condyles up fit very well, with little or no modification, for a majority of the AFMA subjects tested. The distal two-thirds of the socket, however, required considerable modification for all of the test subjects. Similar results were reported in the recent Canadian clinical trials of the Canfit System (19). Because of this, the developers at Vorum Research have recently rewritten the Canfit CASD software, so that the new CanfitPlus System, like the UCL-BC Computer Shape System, is now based on superposition of modification 
templates on digitized residual limb measurements, obtained from either plaster wrap casts or optical scans of patients' residual limbs.

\section{DVA-PRS AFMAp and Shapemaker CASD software development}

As an extension of their work in the National AFMA Program, the PRS investigators developed the AFMAp computer software. The AFMAp software was written as a tool to facilitate identification and quantitative visualization of volumetric differences between residual limbs and prosthetic sockets. AFMAp utilizes a least mean square algorithm to align and minimize the distance between two superimposed objects, viz., a residual limb and socket (20). Under the AFMAp algorithm, the distance is computed between each point of the digitized model of a residual limb and the nearest point on a corresponding socket model, and the objects are automatically aligned so as to minimize the mean of all of the distances squared. After alignment, any nonzero distances between points on the two models are used to generate a "difference" map. The direction and magnitude of the residual distances of the difference map are encoded as colors and displayed as a color map on the digitized model (21). Because of its formidable ability to aid in visualization of complex geometric relationships, the AFMAp software has proven to be a valuable research tool, a clinical resource, and an educational aid, contributing to the understanding and teaching of the biomechanics of residual limb/prosthetic socket design and fit.

A further outgrowth of the National AFMA Program was development of the DVA-PRS Seattle Shapemaker CASD System. The PRS investigators utilized the knowledge they gained in the AFMA outreach, laboratory, and clinical testing programs to derive specifications for an advanced CASD system. The Shapemaker CASD System which they subsequently developed was created on the Apple Macintosh computer as a generic prostheticsorthotics sculptural design and modification program with an extensive range of features and capabilities (Figure $\mathbf{5 4}$ and Figure 55). During its development, the researchers at the NU-VALMC and NY DVAMC AFMA Centers, and prosthetists at several other institutions and facilities, tested the Shapemaker software and provided feedback to the PRS investigators. Because of this, the Shapemaker
CASD system that was developed is a powerful and versatile, user-oriented tool, enabling prosthetists to fully utilize their knowledge and skills to provide improved care for their patients $(22,23)$. In addition, Shapemaker has served as a catalyst for CASD system development, prompting other developers to improve and upgrade their CASD systems, incorporating many of the advanced features and capabilities first developed and included in Shapemaker.

\section{CONCLUSIONS}

The amount of training, experience, and skill of the AFMA prosthetists varied considerably. Sixtyone percent had college degrees, and the highest level of formal education of the remaining 39 percent was high school. The clinical experience of the AFMA prosthetists ranged from recent graduates with slightly more than 1 year of experience, to highly skilled prosthetists with more than 25 years of experience. Fifty-eight percent were $\mathrm{ABC}$ certified. Fifty-eight percent of the AFMA prosthetists had some prior computer experience, but 42 percent had none. Despite this, all of the prosthetists who participated were able to learn to use the CASD/CAM systems and were able to develop at least some degree of proficiency with them. Prosthetists with little or no technical background or prior computer experience took longer to learn to use the systems, in general, but their final ability and proficiency with the systems depended more on the extent of their experience and their prosthetics skills than on the degree of their technical background or prior training.

As previousiy described and as shown in Tables $1 \mathrm{a}$ and $\mathbf{1 b}$, the patients enlisted as test subject had a broad range of medical, physiological, biomechanical, and prosthetics characteristics. This diversity in the test subjects, together with the diversity in the AFMA prosthetists, is felt to be reasonably representative of the prosthetics field in the U.S., and therefore to provide a degree of generality to the AFMA test results. With a conservative interpretation, the AFMA test results at least serve to establish the feasibility of utilizing CAD/CAM systems in clinical settings in the United States for design and manufacture of lower limb prostheses. From a broader perspective, the AFMA 
Journal of Rehabilitation Research and Development Vol. 29 No. 4 Fall 1992

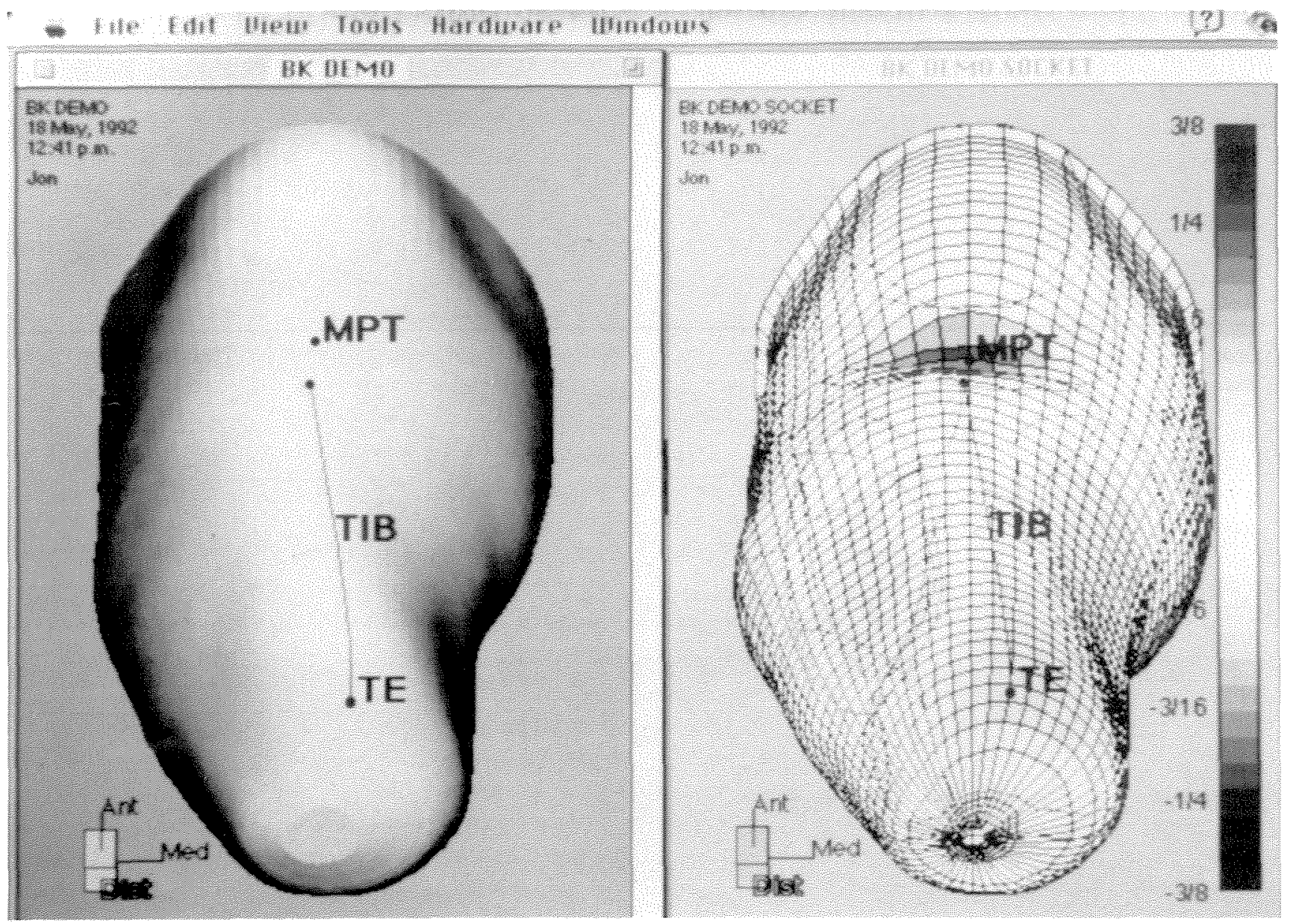

Figure 54.

Demonstration of DVA-PRS Shapemaker CAD System's graphical user interface. Three-dimensional shaded solid model of a residual limb and the wireframe model of a corresponding CASD socket are displayed in two Shapemaker windows. The CASD template modification regions shown on the surface of the wireframe socket model are linked to the residual limb landmarks: MPT, mid-patellar tendon; TIB, tibial crest; TE, end of tibia; FH, fibular head; and FE, end of fibula. The scale at the right of the wireframe window encodes as color (grayscale) hues the "difference map" (mean squared radial distance differences) between the nodal surface elements of the residual limb digitized measurement model and the corresponding socket model, for visualization of the effects of socket design geometries on residual limb loading and tissue displacements.

test results also provide indications of the rates of success and productivities that can be achieved clinically with the UCL-BC and UBC-MERU CASD/CAM systems.

Under the DVA RR\&DS National AFMA BK CASD/CAM Testing and Evaluation Program, 66 prosthetists, 198 patients, and the associated rehabilitation health care staffs in 44 institutions and prosthetics facilities across the United States participated in CASD/CAM outreach trials. In these trials, 281 CASD sockets were designed and fabricated for patients at participating institutions and facilities, with an average turnaround time of 2.6 days, with
46 percent being designed, fabricated, and shipped back to the participating facility in one day or less. As such, the outreach trials achieved the objective of introducing a wide range of prosthetists, physicians, and rehabilitation health care professionals to CAD/CAM technologies, while at the same time demonstrating the vast distributed manufacturing capabilities and productivities achievable with $\mathrm{CAD}$ / CAM technologies in prosthetics.

As the CASD/CAM systems tested in the AFMA Program were first-generation laboratory/ production prototypes, numerous problems were discovered and diagnosed, solutions worked out, 

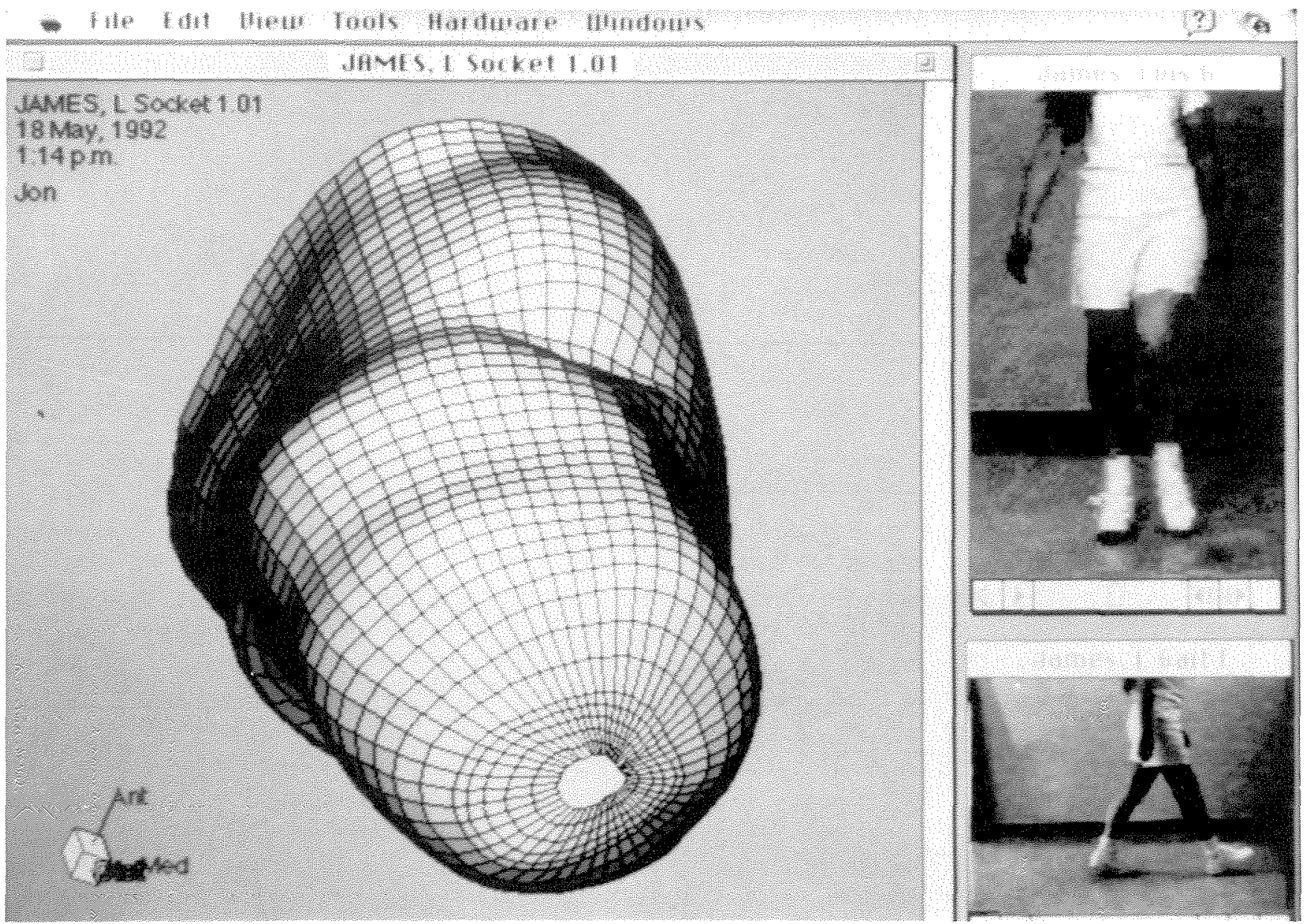

\section{Figure 55.}

DVA-PRS Shapemaker CAD System multiwindow display showing the wireframe model of patient's socket, and frames from the digitized video of the patient ambulating in the socket in a CASD/CAM prosthesis. With additional input of patient residual $\mathrm{limb}$ /socket stress - gait information into Shapemaker, intelligent algorithms can be developed for automatic provision of suggestions for and implementations of socket design modifications for optimization of residual limb/socket loading, function, and fit.

and feedback provided to the system developers. This led to an evolution of the CASD/CAM hardware and software over the course of the AFMA Program. The extensive laboratory tests conducted by the investigators as part of the AFMA Program quantified the performance capabilities and limitations of each component of the CASD/CAM systems tested. Suggestions for improvements arising from these tests led to the refinement of the systems, thereby hastening their transition from prototypes to commercial products, ready for general deployment in clinical prosthetics laboratories. A significant contribution made in the first phase of the AFMA Program was development by the PRS investigators of a new, accurate, repeatable, and reliable electromechanical digitizer for residual limb shape characterization and CASD system input from either plaster wrap casts or existing prosthetic sockets. The PRS digitizer was used in the AFMA Program to replace the original inefficient, less accurate, and less consistent UCL-BC carver mounted potentiometer/follower. It was also subsequently adopted, modified, and made commercially available by the UCL-BC/Shape Products, Ltd. CASD/CAM system developers.

In the carefully controlled clinical tests conducted under the AFMA Program, CASD/CAM sockets were designed, fabricated, and fit on 90 subjects. In these trials, 94 percent of the subjects fit with UCL-BC CASD/CAM sockets and prostheses 
were very to fairly satisfied overall with the quality of their CASD/CAM sockets and prostheses. Six percent of the subjects rated the overall quality of their UCL-BC CASD/CAM sockets as fair to poor. None of the subjects rated their UCL-BC CASD/CAM sockets as very poor. Similarly, 70 percent of the subjects fit with UBC-MERU Canfit CASD sockets rated the overall quality of their CASD/CAM sockets as very good to fair, and 30 percent of the subjects rated the overall quality of their sockets as fair to poor. None of the subjects rated their UBC-MERU Canfit CASD sockets as very poor. By comparison, 80 percent of the subjects rated the overall quality of their conventional pre-AFMA sockets and prostheses as very good to fair, 13 percent as fair to poor, and 7 percent as very poor. In general, the sockets and prostheses produced with the UCL-BC and UBC-MERU CASD/CAM systems did not receive the highest rating by as many subjects as the very best pre-AFMA sockets and prostheses produced using conventional techniques, but more subjects were satisfied with (gave very good to fair ratings to) their CASD/CAM sockets and prostheses than gave very good to fair ratings to their conventionally designed and produced pre-AFMA sockets and prostheses. Furthermore, the worst sockets designed and fabricated with the UCL-BC and UBC-MERU CASD/CAM systems were not rated as bad as the worst conventionally designed and produced preAFMA sockets.

Analysis of the data obtained during the AFMA clinical trials revealed correlations between the amount of work required to achieve successful UCL-BC CASD/CAM socket designs and fits, and the test subjects' residual limb length, durometer of residual limb soft tissue, amount of residual limb soft tissue, activity level, length of time since amputation, and degree of satisfaction with their conventional, pre-AFMA sockets and prostheses. The two factors most prevalent among the subjects most difficult to design and fit CASD sockets successfully for were length of time since amputation and degree of satisfaction with their conventional pre-AFMA sockets and prostheses. Analysis of the AFMA test data showed that older, longerterm amputees, who were quite satisfied with their conventional pre-AFMA sockets and prostheses, required, on the average, 54 percent more work to achieve acceptable CASD/CAM socket designs and fits for, than the other AFMA test subjects. As this class of subjects is usually relatively stable (i.e., their residual limb volume, tissue type, prosthesis fit and functional requirements, muscle strength and endurance, activity level, etc., are relatively constant), they are ideal candidates for CASD/CAM sockets and prostheses. Once successful socket and prosthesis designs and fits have been obtained and are on (computer) file for these subjects, either by digitization of a favorite, existing socket, or through an initial CASD design and fitting process, production of another new socket and prosthesis can be accomplished far more accurately, efficiently, and expediently using CASD/CAM techniques, than with conventional, manual prosthetics procedures. The AFMA investigators felt that the long worktimes required by the difficult to fit subjects, and also to a lesser extent, the work-times required by the other AFMA subjects, could have been significantly reduced: 1) if the UCL-BC CASD system had been designed so that socket template modifications were linked to specific regions uniquely identified and accurately located by residual limb landmarks included at the time of casting and digitization in the subjects' measurement files, and 2) if the UCL-BC CASD system had included general modification capabilities: a) to rapidly create new modification regions, and to adjust all modification regions" locations, shapes, sizes, and topographies; and, b) to compute and apply the net resultant modifications at all socket nodes in such a manner as to ensure linearity of sequential design iterations and smooth transitions at all modification region boundaries.

The success rate achieved in the AFMA clinical trials is believed to validate the design philosophy on which the UCL-BC CASD/CAM system is based, specifically, modification template superposition on digitized measurements of patients' residual limbs. Nevertheless, the relatively large number of template region foci and individual socket node radii that required additional modification before successful socket designs and fits were achieved, together with the large work-times required to effect these modifications, indicate the UCL-BC socket modification templates evaluated in the AFMA clinical trials should be refined and/or new templates developed, especially for subjects with short residual limbs. As part of the AFMA Program, quantitative data were compiled on the test subjects, their medical, physio- 
logical, biomechanical, and prosthetics characteristics, and the CASD/CAM sockets and prostheses designed for and fit on them. These data are being used by the AFMA investigators to develop new CASD socket templates. An additional benefit of the extensive database compiled under the AFMA Program is expansion of the general statistical information about the BK amputee population in the United States. Subsequent analysis of this database has also provided the AFMA investigators with new insights regarding the biomechanics of socket design, fit, and comfort, adding to the basic knowledge available in this area. Suggestions for areas of further CAD/CAM research and development have also been obtained from the analysis of the AFMA database.

A significant achievement of the AFMA Program was development of the DVA-PRS Seattle Shapemaker CASD System. The Shapemaker CASD System was designed and developed by the PRS investigators to be a versatile, user-friendly, generic prosthetics-orthotics sculptural design and modification tool, incorporating the features and capabilities the AFMA investigators found to be important or felt would have been helpful during the AFMA clinical trials. Many of the features first included in the Shapemaker System have begun to be incorporated in other CASD/CAM systems, so that a second generation of improved and enhanced prosthetics CASD systems is now available.

Because of the knowledge gained and the developments made during the DVA National AFMA Program, it is felt that prosthetics CAD/CAM research can now be concentrated in the areas of: 1) developing new, more expedient and comprehensive methods and devices for patient characterization for $\mathrm{CAD} / \mathrm{CAM}$ system input; 2) extending CASD system design capabilities and templates to all levels of amputation; 3) further automating the prosthesis manufacturing process, including extension of CAM methods to structural and cosmetic finishing, with eventual, direct prosthesis manufacture from $C A D$ files through rapid prototyping CAM technologies, such as fused deposition modeling, stereolithography, or selective laser sintering; 4) increasing knowledge of and developing devices for quantitative assessment of socket/ prosthesis fit and alignment; 5) developing methods and devices for quantitative assessment of the mechanical, circulatory, and metabolic residual limb tissue properties of patients, and increasing the understanding of the effects of prosthetic socket stresses on these properties; and, 6) applying computer-aided engineering methods, such as finite element analysis and stress distribution optimization, to achieve prosthesis designs matched to the characteristics and requirements of individual patients. As knowledge is gained, and devices and systems are developed in each of these areas, prosthetists will obtain increasingly powerful tools enabling them to provide improved prosthetics care more effectively, efficiently, and expediently for their patients, thereby helping their patients to live more independent, comfortable, and functional lives.

\section{REFERENCES}

1. Lawrence $R B$, Knox $W$, Coombes AGA, Greenwood CD, Davies RM. The AK-BK Rapidform Socket Fabrication System. London, England: University College of London Department of Mechanical Engineering and Bioengineering Center: 1983 Annual Report. 1983:13-6.

2. Wilson Ir AB, editor. Workshop Group Reports, VASponsored Workshop on Application of Computer-Aided Design and Computer-Aided Manufacturing Techniques to Prosthetics, LaJolla (CA), September 1985.

3. Blocker WP, Krouskop TA. Automated fabrication of lower extremity prosthetic sockets. Rehabil R\&D Prog Rpts-1984. 1984:4-5.

4. Childress DS, Vanderby Jr R, Schnur D, VanVorhis R. Computer-aided analysis of below-knee socket pressure. J Rehabil Res Dev 1985;22(4)16-7.

5. Boone DA, Rappoport AF, Burgess EM. Automated fabrication of mobility aids. Presented at the VA RR\&DS Independence '87 Conference, Washington (DC), March 1987.

6. Boone DA, Burgess EM. Automated fabrication of mobility aids: clinical demonstration of the UCL Computer-Aided Socket Design System. I Prosthet Orthot 1989; 1(3): $187-90$.

7. Wilkinson IC, Dewar MG. The UCL System for Computer-Aided Socket Design: Clinical Results. In: Davies RM, Donovan RG, Spiers RW, editors. Report of ISPO Workshop on CAD/CAM in Prosthetics \& Orthotics. Copenhagen, Denmark: IPSO, 1990: 25-7.

8. Davies RM, Donovan RG, Spiers RW, editors. Report of ISPO Workshop on CAD/CAM in Prosthetics \& Orthotics. Copenhagen, Denmark: ISPO, 1990.

9. Radcliffe $\mathrm{CW}$, Foort J. The patellar tendon bearing below-knee prosthesis. Berkeley, (CA): University of California Press, 1961.

10. New York University Post-Graduate Medical School, Prosthetics-Orthotics Education Program, Lower-Limb Prosthetics With Prosthetists' Supplement. New York: NY Univ. Press, 1990. 
11. Northwestern University Prosthetics-Orthotics Education Program, Below-Knee Prosthetics for Prosthetists. Chicago, (IL): Northwestern Univ., 1985.

12. UCLA Prosthetics-Orthotics Education Program, BelowKnee Prosthetics Manual for Prosthetists. Los Angeles, (CA): UCLA Cont Ed., 1972.

13. Nielson C. Survey of amputees: functional level and life satisfaction, informational needs, and the prosthetist's role. J Prosthet Orthot 1991;3(3):125-9.

14. Hoaglund FT, Jergesen HE, Wilson L, Lamoreux LW, Roberts R. Evaluation of problems and needs of veteran lower-limb amputees in the San Francisco Bay Area during the period 1977-1980. J Rehabil Res Dev $1983 ; 20(1): 57-71$.

15. Kegel B, Carpenter ML, Burgess EM. A survey of lower-limb amputees: prostheses, phantom sensations, and psychological aspects. Bull Pros Res 1977 Spr;1027:43-60.

16. Kegel B, Carpenter ML, Burgess EM. Functional capabilities of lower extremity amputees. Arch Phys Med Rehabil 1978 March;59:109-20.

17. Bennett L, Lee BK. Pressure versus shear in pressure sore causation. In: Lee BK, editor. Chronic ulcers of the skin. New York: McGraw-Hill, 1985:39-56.

18. Bennett L, Kavner D, Lee BK, Trainor FA. Shear vs. pressure as causative factors in skin blood flow occlusion. Arch Phys Med Rehabil 1979 July;60:309-14.

19. Topper AK, Fernie GR. An evaluation of computer-aided design of below-knee prosthetic sockets. Prosthet Orthot Int 1990 Dec;14(3):136-42.

20. Sidles JA, Boone DA. Mathematical techniques for comparing residual limb and socket shapes. Proceedings of the ISPO Sixth World Congress, Kobe, Japan, November 1989.

21. Sidles JA, Boone DA, Harlan JH, Burgess EM. Rectification maps: a new method for describing residual limb and socket shapes. J Prosthet Orthot 1989;1(3):149-53.

22. Boone DA, Harlan JH, Burgess EM. Computer assisted prosthetic design methods. Proceedings of the ISPO Sixth World Congress, Kobe, Japan, November 1989.

23. Boone DA, Harlan JH, Burgess EM. Mathematical techniques for comparing residual limb and socket shapes. Proceedings of the ISPO Sixth World Congress, Kobe, Japan, November 1989. 